\title{
Genetic background and impact of Staphylococcus aureus in a cross-border region
}

Citation for published version (APA):

Nulens, E. F. L. (2010). Genetic background and impact of Staphylococcus aureus in a cross-border region. [Doctoral Thesis, Maastricht University]. Universitaire Pers Maastricht. https://doi.org/10.26481/dis.20101209en

Document status and date:

Published: 01/01/2010

DOI:

10.26481/dis.20101209en

Document Version:

Publisher's PDF, also known as Version of record

\section{Please check the document version of this publication:}

- A submitted manuscript is the version of the article upon submission and before peer-review. There can be important differences between the submitted version and the official published version of record.

People interested in the research are advised to contact the author for the final version of the publication, or visit the DOI to the publisher's website.

- The final author version and the galley proof are versions of the publication after peer review.

- The final published version features the final layout of the paper including the volume, issue and page numbers.

Link to publication

\footnotetext{
General rights rights.

- You may freely distribute the URL identifying the publication in the public portal. please follow below link for the End User Agreement:

www.umlib.nl/taverne-license

Take down policy

If you believe that this document breaches copyright please contact us at:

repository@maastrichtuniversity.nl

providing details and we will investigate your claim.
}

Copyright and moral rights for the publications made accessible in the public portal are retained by the authors and/or other copyright owners and it is a condition of accessing publications that users recognise and abide by the legal requirements associated with these

- Users may download and print one copy of any publication from the public portal for the purpose of private study or research.

- You may not further distribute the material or use it for any profit-making activity or commercial gain

If the publication is distributed under the terms of Article $25 \mathrm{fa}$ of the Dutch Copyright Act, indicated by the "Taverne" license above, 
Genetic background and impact of Staphylococcus aureus in a cross-border region 
(c) Eric Florent Leopold Nulens, Brugge, 2010.

All rights reserved. No part of this thesis may be reproduced or transmitted in any form, by any means, electronic or mechanical, without the prior written permission of the author, or where appropriate, of the publisher of the articles.

\section{ISBN}

9789461590244

Lay-out

(C) Eric Nulens, Josine Bielen.

\section{Fotos}

(C) Eric Nulens, Josine Bielen. 


\title{
Genetic background and impact of Staphylococcus aureus in a cross-border region
}

\author{
PROEFSCHRIFT \\ ter verkrijging van de graad van doctor \\ aan de Universiteit Maastricht, \\ op gezag van de Rector Magnificus, \\ Prof. mr. G.P.M.F. Mols \\ volgens het besluit van het College van Decanen, \\ in het openbaar te verdedigen \\ op donderdag 9 december 2010 om 16.00 uur \\ door
}

Eric Florent Leopold Nulens

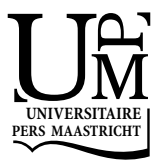


Promotiecommissie

\section{Promotor}

Prof. dr. C.A. Bruggeman

\section{Copromotor}

Dr. E.E. Stobberingh

\section{Beoordelingscommissie}

Prof. dr. M.H. Prins (voorzitter)

Dr. C.J.P.A. Hoebe

Prof. dr. G. leven (Universitair Ziekenhuis Antwerpen, België)

Prof. dr. R. Koopmans 


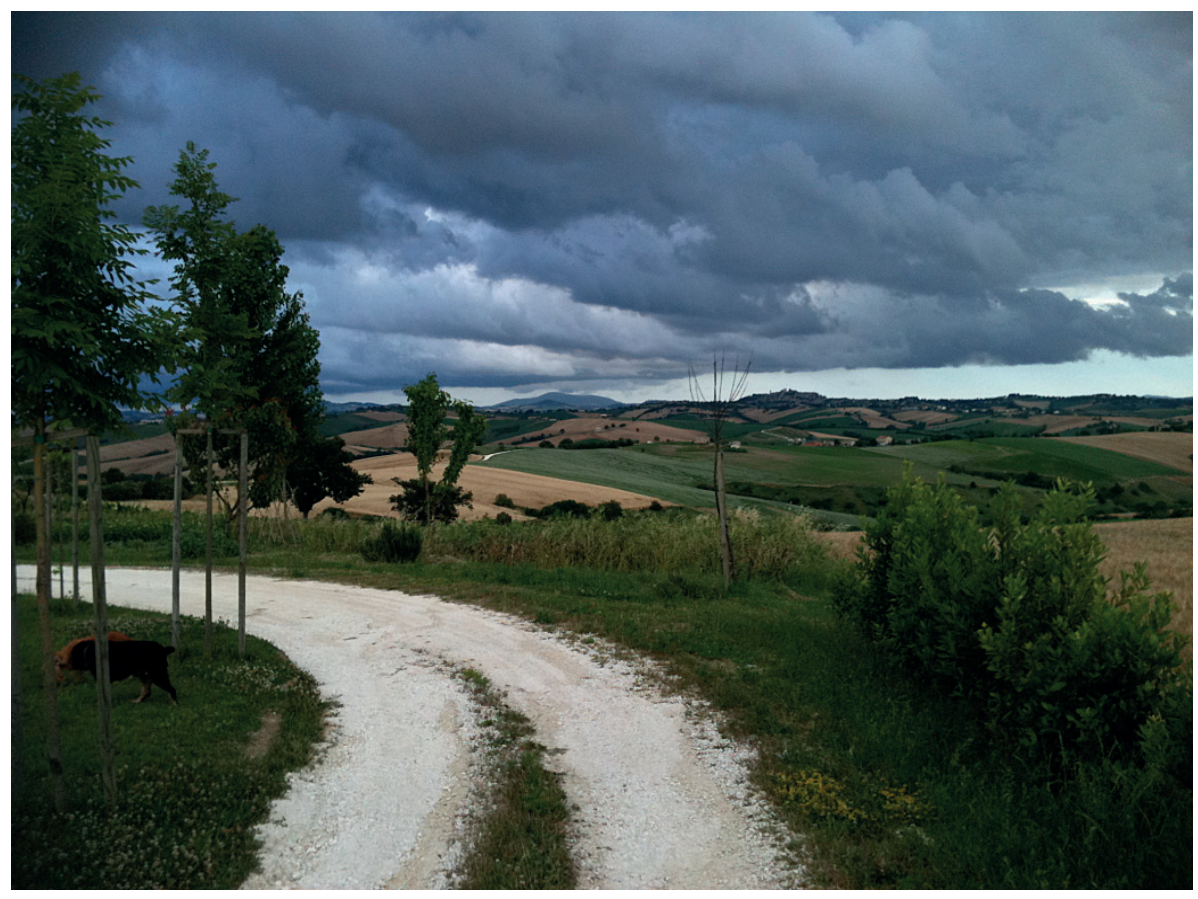

Cingoli, 2010 



\section{Contents}

Abbreviations 8

$\begin{array}{ll}\text { Map of the Euregion Meuse-Rhine } & 11\end{array}$

$\begin{array}{lll}\text { Part } 1 & \text { Introduction } & 13\end{array}$

$\begin{array}{lll}\text { Chapter } 1 \text { General introduction } & 15\end{array}$

Chapter 2 Outline of the thesis 39

Part 2 Genetic background and impact of Staphylococcus aureus in a $\quad 43$ cross-border region

Chapter 3 Molecular Characterisation of Staphylococcus aureus 45 Bloodstream Isolates Collected in a Dutch University Hospital between 1999 and 2006

Chapter 4 Genetic diversity of methicillin-resistant Staphylococcus aureus in a tertiary hospital in The Netherlands between 2002 and 2006

Chapter 5 Cross-Border Dissemination of Methicillin-Resistant Staphylococcus aureus, Euregio Meuse-Rhine Region

Chapter 6 Cost of the meticillin-resistant Staphylococcus aureus search and destroy policy in a Dutch university hospital

Chapter 7 Contribution of Two Molecular Assays as Compared to Selective 103 Culture for MRSA Screening in a Low MRSA Prevalence Population

\section{Part 3 Discussion}

Chapter 8 Discussion

Chapter 9 Summary 


\section{Abbreviations}

\begin{tabular}{|c|c|}
\hline bp & base pair \\
\hline BSI & bloodstream infection \\
\hline BURP & based upon repeat pattern \\
\hline CA & community-associated \\
\hline CA-MRSA & community-acquired MRSA \\
\hline CC & clonal complex \\
\hline$c c r$ & cassette chromosome recombinase \\
\hline $\mathrm{CF}$ & cystic fibrosis \\
\hline CFU & colony-forming units \\
\hline $\mathrm{Cl}$ & confidence interval \\
\hline CLSI & clinical laboratory standards institute \\
\hline CNA & collagen adhesion \\
\hline $\mathrm{D}$ & decolonization cost \\
\hline DI & index of diversity \\
\hline EMR & Euregion Meuse-Rhine \\
\hline FDA & food and drug administration \\
\hline HA & hospital-associated \\
\hline HA-MRSA & hospital-acquired MRSA \\
\hline HCWs & healthcare workers \\
\hline HOS & hospitalisation cost \\
\hline Ic & additional isolation cost for colonized patients \\
\hline In & additional isolation cost for patients with MRSA BSI \\
\hline LOS & length of stay \\
\hline MIC & minimal inhibitory concentration \\
\hline MLST & multi-locus sequence typing \\
\hline MLVA & multi-locus variable tandem analysis \\
\hline MRCNS & methicillin-resistant coagulase negative staphylococci \\
\hline
\end{tabular}




$\begin{array}{ll}\text { MREJ } & \text { SCCmec right extremities junction } \\ \text { MRSA } & \text { methicillin-resistant Staphylococcus aureus } \\ \text { MSSA } & \text { methicillin-susceptible Staphylococcus aureus } \\ \text { MUMC } & \text { Maastricht University Medical Center } \\ \text { NC } & \text { cost of negative MRSA culture } \\ \text { PBP } & \text { penicillin binding protein } \\ \text { PC } & \text { cost of positive MRSA culture } \\ \text { PCR } & \text { polymerase chain reaction } \\ \text { PFGE } & \text { pulsed-field gel electrophoresis } \\ \text { PVL } & \text { Panton-Valentine leukocidin } \\ \text { RT-PCR } & \text { real time polymerase chain reaction } \\ \text { SCCmec } & \text { Staphylococcal Cassette Chromosome mec } \\ \text { S\&D policy } & \text { search and destroy policy } \\ \text { spa-CC } & \text { spa-clonal complex } \\ \text { SSR } & \text { short sequence repeat } \\ \text { ST } & \text { sequence type } \\ \text { TAT } & \text { turn-around time } \\ \text { Tf } & \text { treatment cost of MSSA BSI } \\ \text { TSST-1 } & \text { toxic shock syndrome toxin-1 } \\ \text { TV } & \text { treatment cost of MRSA BSI } \\ \text { UHM } & \text { University Hospital Maastricht } \\ \text { WIP } & \text { workingparty on infection prevention } \\ & \end{array}$





\section{Map of the Euregion Meuse-Rhine}

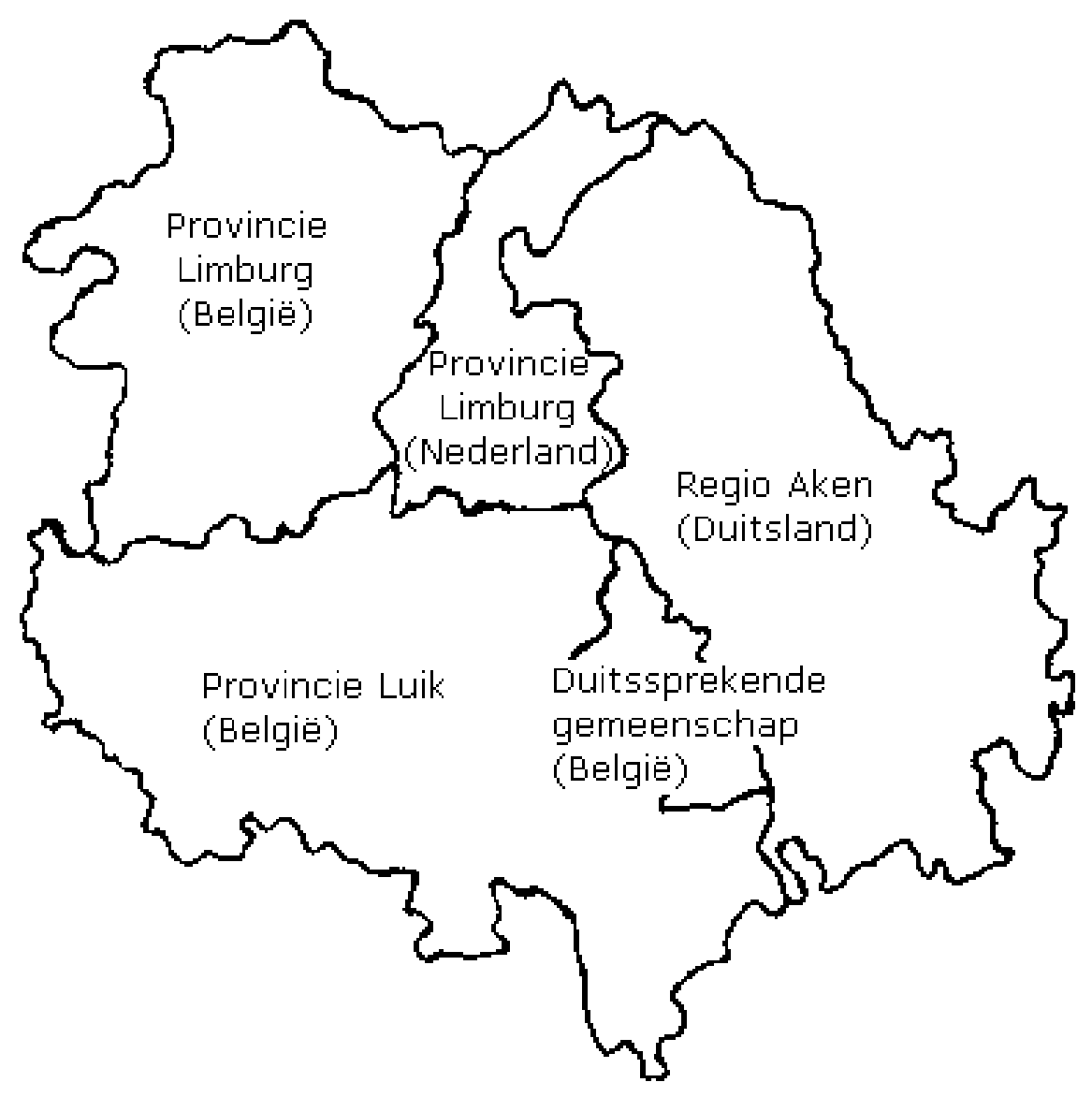





\section{Part 1: INTRODUCTION}

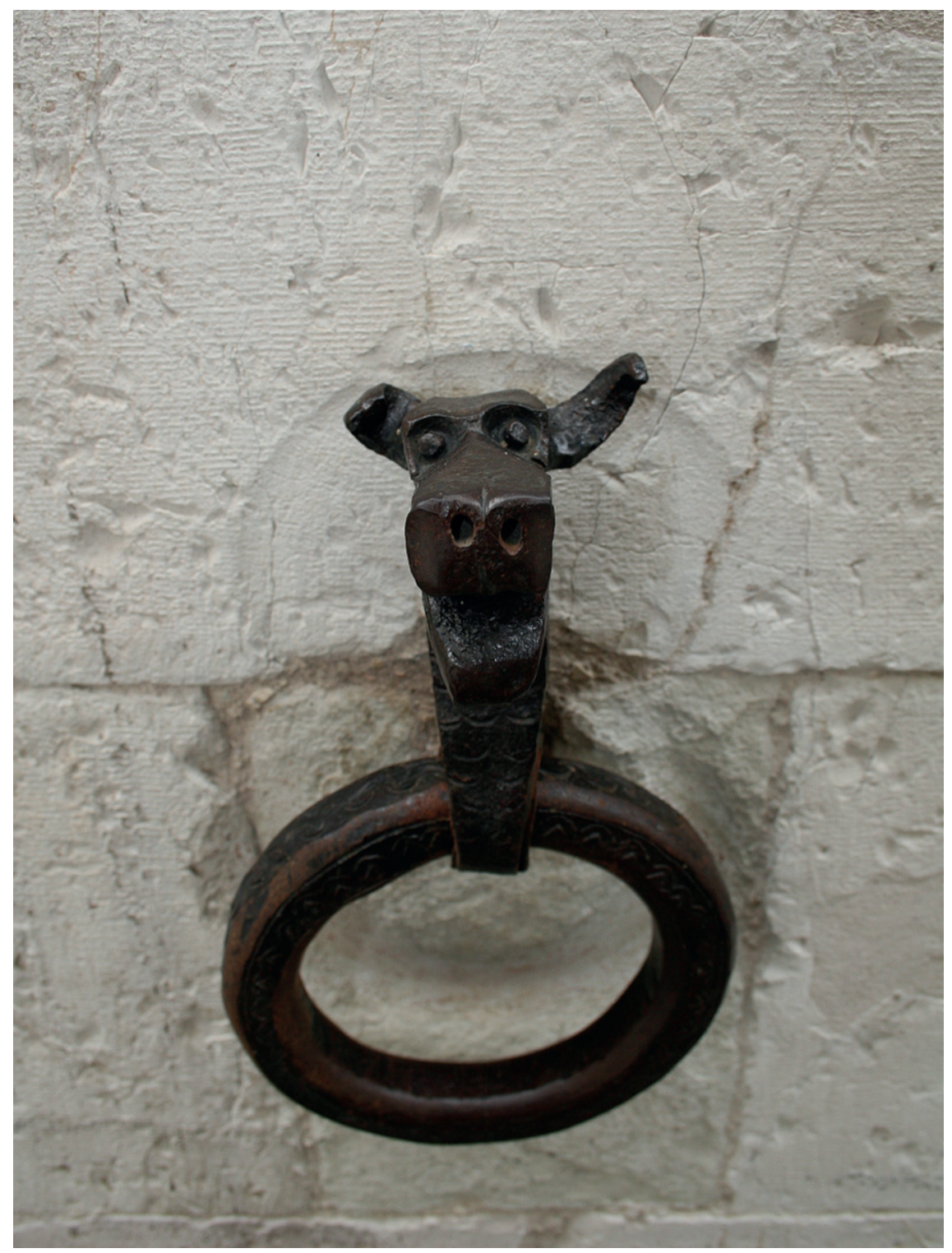

Horse's parking ornament, Umbria, 2009 



\section{Chapter 1: General introduction}

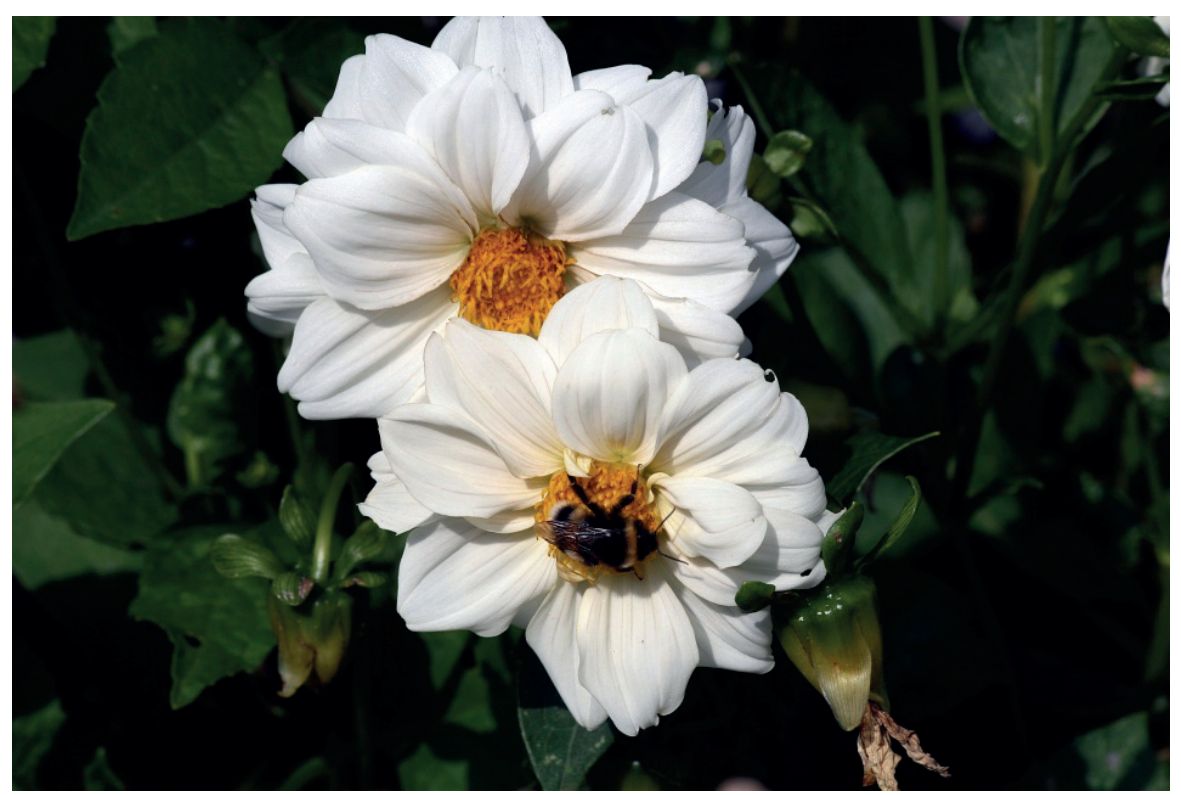

Dahlia, Brugge, 2009 


\section{General Introduction}

In 1882, Alex Ogston, a Scottish surgeon was the first who described the clinical manifestations, the inflammatory processes and septico-pyaemic conditions caused by Micrococcus. Two distinct forms were seen, the chain coccus, streptococcus and the grouped form staphylococcus. The behaviour of staphylococcus on the intact skin was completely different compared to experimentally subcutaneously injected animals or in human cases. He observed the differences in the organs or structures invaded, the varying virulence of the organism and that susceptibility of the individual may play a role in the pathogenesis of the organism [50].

It was however until 1929 that penicillin was isolated from Penicillium notatum by Fleming, and introduced in clinical medicine in 1941 for the treatment of infections caused by Staphylococcus aureus. Shortly after the introduction of penicillin, the bacteria became resistant to this agent due to the acquisition of a plasmid coding for a beta-lactamase. Currently about $80 \%$ of all S. aureus isolates are resistant to penicillin. Methicillin, a penicillinase resistant beta-lactam antibiotic was introduced in 1960 for the treatment of S. aureus infections. Already in 1962, methicillin-resistant $S$. aureus strains were isolated in the United Kingdom and Denmark. From that moment on, 5 major methicillin-resistant $S$. aureus (MRSA) clones disseminated through Europe and the rest of the world [13].

\section{Staphylococcus aureus}

S. aureus belongs to the indigenous flora of the skin and the mucosa of humans and is a common causative agent of community and hospital-acquired infections. About $20 \%$ of community dwellers are permanently colonised by $S$. aureus mainly in the nose. Several other sites may also be colonised including the throat, the gastro-intestinal tract, the perineum, and the axillae. Another $30 \%$ is transient carrier and $50 \%$ is never carrier $[34,45,72]$. Children have a higher persistent carrier state than adults, and rates may change from persistent to transient carriers during adolescence [72]. Why some individuals are permanently colonised while others are not, has not been cleared in full. Probably repeated exposure to $S$. aureus, genetic, bacterial and physiological factors determine the patient susceptibility to $S$. aureus colonisation $[42,72]$.

In the community S. aureus is a frequent cause of skin infections, like folliculitis, furunculosis, and impetigo, and in $80 \%$ patients are nasal carriers. Sometimes infections may become invasive and give rise to more devastating infections such 
as endocarditis, osteomyelitis, and prosthetic infections. By the time patients get to the hospital they are in a bad clinical condition with shock and their clinical outcome is affected [42].

S. aureus is the most common cause of hospital-acquired or nosocomial infections. In the majority of the patients, these infections are caused by the endogenous $S$. aureus isolates, because persistent carriers have a 3 to 6 times increased infection risk compared to non-carriers, due to dispersal of higher nasal S. aureus loads [2, 72]. Dialysis, surgery, cancer, lung disease, diabetes mellitus, and intravascular catheters are important risk factors in developing $S$. aureus bloodstream or invasive infections $[3,37,42,43,73]$. Patients with a disseminated $S$. aureus infection have a higher mortality risk than those with an uncomplicated infection, which in turn have a higher risk than patients with other nosocomial infections or without any infection $[42,43]$.

Mammals have developed defences which have been shaped by co-evolution with S. aureus, such as physical barriers of mucus covered epithelial layers, secretion of antimicrobial factors, and the normal immune response. However, $S$. aureus may initiate various virulence factors in order to facilitate survival, and to cause invasive infections [73]. Structural virulence factors, such as clumping factors, and secreted virulence factors, for instance leucocidins, proteases, lipases and enterotoxins, play a role in the pathogenesis of $S$. aureus infections. Surface proteins initiate infection by adhesion to host tissues components, like collagen, fibronectin and fibrinogen. Than S. aureus will grow and persist in host surfaces in various ways, by forming biofilms, by invading epithelial cells or, by forming small colony variants in host cells. The host immune system is evaded by an antiphagocytic pathway, and by protein A, which bind to the Fc portion of immunoglobulins. Due to the production of proteases, lipases and elastases, invasion and destruction of host tissue and metastatic dissemination to other sites is established. However there is a marked difference in the capability of $S$. aureus strains in their adhesive power, the production of toxins, biofilm formation, and resistance to phagocytosis. Furthermore the distribution of certain virulence factors seems to be related to particular clonal types $[10,23$, 73]. The process of adherence, persistence and evading the immune system is under control of signalling mechanism of $S$. aureus called quorum sensing. Quorum sensing allow bacteria to detect the density of their own population in a certain anatomical site, and to alter the genetic expression virulence genes in order to select a genetic advantage [10]. 


\section{Methicillin-resistant Staphylococcus aureus}

Resistance to methicillin and all beta-lactams antibiotics is due to the acquisition of the mecA gene, which codes for the Penicillin Binding Protein (PBP) 2a. This enzyme is responsible for the peptidoglycan synthesis, which is part of the cell wall of S. aureus, and contrary to other PBPs has a low binding affinity for betalactam antibiotics $[35,51]$. The mecA gene is located on a mobile genomic island, the Staphylococcal Chromosome Cassette ( $\mathrm{SCCmec),} \mathrm{which} \mathrm{is} \mathrm{integrated} \mathrm{at} \mathrm{a}$ specific site, the SCCmec attachment site (attBscc) at the $3^{\prime}$ end of an open reading frame (orfX) of the $S$. aureus genome. Currently eight main $\mathrm{SCCmec}$ types are described (I-VIII) [30]. SCCmec types I, IV, V, VI and VII are smaller in size than the other SCCmec types, and cause resistance to beta-lactam antibiotics only. In contrast, SCCmec types II and III may cause resistance to other antibiotics, such as aminoglycosides, macrolides and tetracyclines, due to plasmids and transposons integrated in the SCCmec. The recently described novel SCCmec type VIII isolated in a MRSA in a Canadian hospital-acquired MRSA isolate is comparable to SCCmec type II [30,34]. In addition $S$. aureus may also carry resistances genes to other antibiotics at other sites of the chromosome [14].

Genes responsible for the regulation of the transcription of mecA gene are located on mec complexes. So far 5 major classes of mec complexes have been distinguished (A-E) of which $A$ and $C$ are the most common in the SCC elements [13]. The mecA gene is regulated by the repressor Mecl and the transmembrane beta-lactam-sensing signal-transducer MecR1. In the absence of beta-lactam antibiotics, Mecl represses the transcription of mecA and mecR1-mecl. However in the presence of beta-lactam antibiotics MecR1 is auto catalytically cleaved, and this activates a process which ends by binding of $\mathrm{Mecl}$ to the mecA operator region, allowing the transcription of $m e c A$, and the production of PBP2a.

For integration and excision of the $\mathrm{SCCmec}$ into the $S$. aureus chromosome, chromosome recombinases (ccr) are essential and their genes are also located on the SCCmec. Both the mec complex and the ccr genes are essential to determine the $\mathrm{SCCmec}$ type of an isolate. All SCCmec elements are further divided in three regions. Regions bordering the mec and ccr complexes are designated the joining regions (J). In SCCmec types I-IV several variants in the J regions have been described. $\mathrm{J} 1$ is the region between the right chromosomal junction and the car complex, $\mathrm{J} 2$ is between the ccr gene complex and the mec gene complex, and $\mathrm{J} 3$ is between the mec complex and the left chromosomal junction. The regions consist of non-essential components, but may also carry additional antimicrobial resistance determinants $[13,30]$. 
The initial event that a methicillin-susceptible $S$. aureus (MSSA) became a MRSA isolate has so far not yet been elucidated. It is believed that horizontal DNA transfer of the mecA gene from a Staphylococcus sciuri strain to $S$. aureus has taken place, because of the high percentage of amino acid sequence homology between the Protein Binding Protein (PBP) from S. sciuri and PBP2a from MRSA. Since the isolation of the first MRSA isolate in the United Kingdom in 1961, MRSA has spread worldwide. In most countries the prevalence of MRSA in hospitals now exceeds $20 \%$ to $50 \%[3,6,24]$.

At first, the hypothesis was that a single clone was responsible for the MRSA epidemic, but several studies support now the multi-clone theory: SCCmec was introduced several times into MSSA lineages resulting in a limited number of MRSA clones with their own genetic background disseminating worldwide [54]. This hypothesis was further supported by Enright et al. in 2002, when they identified 11 major epidemic MRSA clones using MLST, and SCCmec typing on a large international collection of MRSA and MSSA isolates [17]. They suggested that only a small number of MSSA isolates had the ability to acquire the methicillin-resistance gene, and that these strains could maintain high epidemicity in the population $[34,44]$.

Genetic typing showed that epidemic MSSA strains from the 1950s with the MSSA ST250 genotype, probably evolved from the MSSA ST8 type, which preceded the ST250-MRSA-I strains, now known as the so called Archaic MRSA clone. The 5 major pandemic clones now disseminated into hospitals worldwide, are the New-York/Japan clone (ST5-MRSA-II), the Brazilian clone, the Hungarian clone, the Paediatric clone, and the Iberian clone (ST247-MRSA-I). The major MRSA clones differ from each other by six or seven MLST loci, and are associated with $\mathrm{CC} 5, \mathrm{CC} 8, \mathrm{CC} 22, \mathrm{CC} 30$ or $\mathrm{CC} 45$. In addition to the dissemination of multiple MRSA clones the acquisition of SCCmec was four times more common than the replacement of a SCCmec element in MRSA [54]. SCCmec type IV is harbored by twice as much MRSA clones, compared to other SCCmec elements, probably due to its smaller size compared to the other types. The small size of SCCmec IV and possible also of SCCmec V may facilitate the transfer of these SCCmec elements between Staphylococci [54]. In a recent European study it was observed that MRSA clones with particular spa types are predominantly regional distributed in contrast to MSSA which shows more diverse spa types [25].

Several MSSA lineages have been observed worldwide with a genetic background that is different from the major MRSA clones, such as CC7, CC9, CC12, CC15, CC25, CC51, and CC101 [14]. These lineages are found in the community and the hospital environment, and are obviously not associated with MRSA clones. The 
observation that more MSSA lineages have been observed compared to MRSA lineages suggest that the MSSA population is more heterogeneous. Some MSSA lineages have a genetic background uncommon to the endemic MRSA clones. This supports the observation that these MSSA lineages may not provide a stable genomic environment for the integration of SCCmec [25, 34]. Also the genetic background of $S$. aureus seems to be important for the stability of SCCmec, because some $S$. aureus lineages lack the ability to integrate $\mathrm{SCCmec}$ into the genome [44]. The epidemiological success of MRSA clones seems therefore dependent on environmental, host associated factors, and intrinsic genetic factors of the bacterium [26].

\section{MRSA detection}

Conventional bacteriological culture is the gold standard for the detection of MRSA, but the technique is laborious and time consuming, and may take up to 5 days before completion. Screening is generally performed by swabbing the anterior nares or at multiple superficial sites, including nares, throat and perineum and other potential sites which may be colonised by MRSA. Nasal screening alone has a sensitivity of about $80 \%$, which may be increased to $95 \%$ by screening at other sites and by overnight enrichment in a broth with sodium chloride $[4,60,77]$.

Initially in-house developed selective media containing oxacillin as selective agent were used for screening patients, but currently cefoxitin is more reliable in detecting resistance [4]. Recently selective chromogenic solid media were introduced for the detection of MRSA in combination with enrichment broth. This chromogenic medium contains a colourless chromogenic substrate which is cleaved by MRSA. The chromogenic end-product becomes than insoluble and accumulates in the growing colony, resulting in a colour change which becomes visible after 24 hours of incubation. Selectivity of the media is obtained by the addition of cefoxitin or oxacillin. However, chromogenic media with oxacillin require 48 hours of incubation to detect MRSA [4, 41]. MRSA is detected in 20-48 hours with most of these media, and MRSA negative results are usually reliable after 24 hours of incubation.

Recently non-culture techniques were developed to detect MRSA directly in patient samples. The turn-around time (TAT) of these molecular and nonmolecular techniques is substantially shorter than culture techniques. The best known assays, the Xpert ${ }^{\mathrm{TM}}$ MRSA assay and BD GeneOhm ${ }^{\mathrm{TM}}$ MRSA assay are two similar real-time PCR's based on the same principle. The right-extremity junction of SCCmec (MREJ) is a single locus that includes the right extremities of SCCmec, 
the SCCmec integration site, and a $S$. aureus specific gene, orfX $[21,29]$. The detection of the MREJ region serves as the basis of current molecular assays. Currently five primers target SCCmec sequences corresponding to types I, II, III, $\mathrm{IVa}, \mathrm{IVb}$, and IVc are used $[15,29]$.

In several publications these assays have been evaluated extensively, usually in a high prevalence MRSA setting. The assays have proven to be useful as a screening assay due to their high negative predictive value $[41,62]$. However molecular methods may give false-positive results, because the mecA gene is only detected circumstantially, because its presence is associated with the detection of these right extremities of SCCmec. Therefore, false-positive results are encountered if non-specific sequences are amplified, if MSSA isolates contain small fragments of the right extremity of $\mathrm{SCCmec}$, or if MRSA strains lose their mecA gene but retain partial SCCmec elements $[15,19,33,44,74]$. In a recent publication the use of primers and probes for the simultaneous detection of the spa gene, mecA gene and SCCmec inserted into the $S$. aureus chromosomal attBscc insertion site, gave promising results [75]. False-positive test results regarding the detecting of MRSA in wound and blood culture specimens might occur due to incomplete genetic sequences or after antibiotic treatment $[21,74,75]$. False-negative results may appear if unusual, regional or new SCCmec types appear which are not detected by current used primers, or when patients have received local or systemic antibiotics prior to testing [21]. At the time of the development of the BD GeneOhm $^{\mathrm{TM}}$ MRSA screening assay, seven MREJ typed were described. Actually at least 20 known MREJ types exist and therefore, several of these types may go undetected with current assays [60]. Therefore a regular molecular update by the manufacturers of commercial assays is essential in keeping up with the genetic evolution of MRSA.

These molecular assays are licensed for nasal screening only. However, the use of pooled swabs taken from multiple sample sites, was as accurate as nasal screening alone. Furthermore screening the nares alone may miss more than $20 \%$ of MRSA colonised patients [21, 33, 56, 76, 77].

An advantage of the molecular methods compared to conventional culture is the short processing time of a few hours, and the limited technical skills for performing the tests. On the other hand the TAT may be longer, when after collection, transport time to laboratory reception increases, when there is need to batch the tests for financial reasons, or when no testing is performed outside opening office hours of the laboratory $[59,62,70]$. Another drawback is that the cost of the molecular detection assays is significantly higher than the cost of culture, also because of the relatively high invalid/inhibition rate when testing 
nasal and off-label samples, which requires retesting of the sample [33, 70, 77]. However the use of molecular assays for screening patients may decrease the number of isolation days with $60 \%$, and lower the cost with €95 to €125 per isolation day avoided. Nevertheless in terms of cost, the introduction of molecular tests to screen patients at admittance does not seem to be costeffective [70].

The lower limit of detection of direct culture, with and without enrichment, and the molecular assays is between 9 and $190 \mathrm{CFU} / \mathrm{swab}$. Enrichment and culture seems to be the most sensitive method $[38,41,56,74]$. Applying the combination of enrichment and a molecular assay could further increase the sensitivity, at the expense of the cost and an increased TAT.

There is still a lot of debate whether the use rapid PCR tests have any influence on the transmission of MRSA and infections between HCWs and patients, and between patients. In a recent meta-analysis, four studies showed a decrease in the prevalence of MRSA infections, while another four studies observed no effect when rapid PCR tests were applied [9, 31, 62]. One study reported a decrease in MRSA infections in medical ICUs, but not in surgical ICUs [62]. In these studies, inhouse assays or the GeneOhm ${ }^{\mathrm{TM}}$ assay were used, with a relative long median TAT between 19.3 and 23 hours $[9,62]$.

Most studies using these assays have shown their usefulness in screening and the detection of MRSA in high risk patients. The high specificity compared to culture, and the high negative predictive value can are very useful to consider patients as non-carriers [41]. However, there are only a few studies that evaluated the molecular screening assays in a population with a low MRSA prevalence $[49,70]$. In the latter studies, the negative and positive predictive value were comparable. Thus patients with a negative PCR result can be considered as non-carrier. However, a false-positive result has serious (financial) consequences for the hospital, for the patients and for HCWs, because of unnecessary isolation of noncolonised patients. Studies as to the usefulness of these assays in a low prevalence population are important $[49,62]$. According to good clinical practice, in case of a positive result with a molecular assay directly on patients samples the presence of MRSA should be confirmed with culture [21]. Finally, patient screening assays are part of the infection control policy and must be used in conjunction with isolation procedures. 


\section{S. aureus typing}

As part of outbreak investigation and research tools, a number of techniques have been developed to determine the clonal relationship between isolates. These techniques may be used alone or in conjunction with other typing assays to increase the discriminative power. Except SCCmec typing, the different methods can be used for both MSSA and MRSA typing. Based on these methods MSSA showed a greater genetic diversity than MRSA.

\section{Pulsed-field gel electrophoresis}

Pulsed-Field Gel Electrophoresis (PFGE) is considered the gold standard and is the best known $S$. aureus typing technique. DNA of the isolates is digested with restriction enzymes and the DNA fragments are separated by agarose gel electrophoresis in an alternating electrical field. After electrophoresis, the banding patterns are analysed according to the criteria of Tenover [63]. PGFE has a large discriminatory power, and can be used to investigate outbreaks, including transmission and spread of MRSA in a hospital as well as between hospitals [18]. But the technique could blur long-range evolutionary associations in MRSA strains [57]. Livestock associated CA-MRSA cannot be typed by PFGE because DNA is methylated at cytosine residues and is thus not digested by Smal $[34,68]$. The main disadvantage of the technique is that it is difficult to standardize and therefore comparison of the results between laboratories is not possible.

\section{SCCmec typing}

A SCCmec type of an isolate is determined by the class of the mec complex and the type of ccr gene complex. Currently eight types ranging from 20 to $67 \mathrm{~kb}$ have been described [30]. Different PCR based methods have been developed for typing SCCmec. With this technique different structural properties of SCCmec are determined. Recently, Chongkatrool proposed a new nomenclature of SCCmec elements based on the ccr genes (indicated by a Greek number) and the mec complex (indicated by an uppercase letter). The nomenclature also designates differences in the J regions, and $\mathrm{SCCmec}$ subtypes are based on the polymorphisms in this region $[14,29]$. The J region may contain characteristic genes or non-coding regions, or mobile genetic elements, such as insertions sequences, and plasmids or transposons, usually encoding for additional antimicrobial resistance [29].

In 2002, a universal nomenclature using MLST and SCCmec typing to define unique MRSA clones has been accepted by the International Union of 
Microbiological Societies subcommittee on S. aureus typing. MRSA clones should be named by their MLST, resistance phenotype, and SCCmec type [34].

\section{Multi-locus sequence type}

Multi-Locus Sequence Type (MLST) is a DNA sequence based typing method that relies on analysis of relatively conserved genes [14, 34]. Housekeeping genes are used because they are essential to every organism. For $S$. aureus typing, the sequence of internal fragments (450 bp) of seven household genes is analysed. The genes are selected because they provide the greatest number of alleles and enabled adequate resolution. Each sequence difference of an allele is given a number, and a string of seven numbers then represents the allelic profile of an isolate, or the sequence type (ST). This technique can be used for both MRSA and MSSA isolates and their mutual relationship can be compared. The BURST algorithm utilizes related STs to separate MLST data sets into clonal complexes (CC). This method attempts to represent founding types and their progeny. The founding genotype is the ST with the largest number of variants differing at one locus. Improved colonisation potential or the acquisition of antibiotic resistance gene may give the founding type a relative dominance. Once established the founding type will diversify over time because of the accumulation of mutations and by the occurrence of recombination events. Each MLST type can be compared with the database available online on the www.mlst.net website. The site provides a central point for the rapid designation of new alleles and STs. It also collects additional relevant data on strains isolated worldwide. Therefore, it is possible to compare information from different genotypes worldwide. By contrast to PFGE, MLST sequence variations accumulate slower than variations in PFGE, since they arise from neutral mutations in housekeeping genes [34]. As the resolution of MLST is too low the method is not appropriate as primary typing method in a routine laboratory, nor as short-term epidemiological research tool. The method is useful in population analysis studies [52, 53, 59, 76]. A disadvantage of MLST typing is the economic and time-consuming cost of the technique $[27,59]$.

\section{Multi-locus Variable Tandem Analysis (MLVA)}

Repetitive DNA occurs in large quantities in eukaryotic organisms and in prokaryotes. The genome of $S$. aureus harbors a variety of DNA repeats, and their in-gene location is regularly updated. They vary in size, localisation, complexity and repeat mode. Individual repeat units may be introduced or deleted during DNA polymerase transcription, and is dependent of the accuracy of the DNA repair system. These repeat changes may affect genome function, but why this 
happens has not been explained so far. It has been suggested that repeat variation offers selective advantage in certain circumstances [39, 65]. MLVA typing of $S$. aureus is a recently developed PCR based typing method. Based on the variations in the number of repeats in seven individual genes, localised on the genome of $S$. aureus, different types can be distinguished [39, 40, 65]. This technique has a discriminatory power comparable with that of PFGE and both methods are highly concordant in terms of discerning clusters of related isolates [53]. Technically MLVA generates reproducible results, is faster, less expensive and more easily applicable in clinical laboratories than PFGE. Therefore MLVA is useful for the application of short-term epidemiological investigations of outbreaks [53].

\section{spa typing}

This single locus sequence based typing method uses the sequences of the polymorphic region $\mathrm{X}$ of the $S$. aureus protein $\mathrm{A}(s p a)$. This repetitive region contains a varying number of 24 base pair (bp) repeats, because the number and sequence of individual repeats may differ [20]. Its diversity is attributed to deletions, duplications, and point mutations. [27]. The spa types are assigned through the Ridom SpaServer and clustered into spa-Clonal Complexes (spa-CC) using the algorithm based upon repeat patterns (BURP) with the software package StaphType (Ridom GmbH, Würzburg, Germany) [14, 18]. A universal spa nomenclature of the typing data is assured by the Seqnet.org initiative to which the typing data are synchronised. The database comprised more than 3900 spa types of about 230 spa repeats from countries worldwide [14, 18]. Several spa types correspond to a single ST, but the spa types remain within an assigned clonal cluster $[14,17]$. The technique may be used for short and long term epidemiological investigations in outbreak, and spa-CC data are in concordance with MLST typing results $[18,48,76]$.

For MRSA and MSSA typing, spa typing has similar levels of discriminatory powers compared to PFGE typing [18, 52]. However, spa typing/BURP may lack discriminatory power, due to the presence of related spa loci in different clonal lineages because of recombination events. The combination with other genetic typing methods may overcome the shortcomings by spa typing alone [14, 52]. This technique has a high throughput, and full portability of data, due to its good reproducibility. 


\section{Community-acquired MRSA}

During the last decade community-acquired MRSA (CA-MRSA) has emerged worldwide also in countries with a traditional low prevalence of hospital-acquired MRSA (HA-MRSA) such as the Netherlands and the Scandinavian countries [14]. Healthy patients without any known MRSA risk factor developed skin and soft tissue infections and necrotising pneumonia, sometimes with devastating consequences $[22,58]$.

The Centers for Disease Control and Prevention defines a CA-MRSA infection as "an infection in a non-hospitalised patient, without any history of MRSA colonisation or infection, without any known history of hospitalisation or admission in a health care facility, or without the presence of a permanent urinary catheter and dialysis patients. CA-MRSA isolates are in contrary to HospitalAcquired-MRSA (HA-MRSA), often susceptible to non-beta-lactam antibiotics, probably because CA-MRSA isolates carry the smaller SSCmec type IV" [14].

The prevalence of CA-MRSA in the general population is low, but several publications suggested an increase [14]. In a meta-analysis by Salgado however, in which the CA-MRSA prevalence in different studies were compared, most CAMRSA isolates were derived from individuals with at least one risk factor for MRSA. Therefore, it was concluded that the prevalence of CA-MRSA in the community so far is low [58]. However, during recent years, CA-MRSA has started to replace HA-MRSA in health care facilities, especially in the USA and Taiwan, where the CA-MRSA prevalence is higher than in Europe, and therefore the distinction between CA-MRSA and HA-MRSA is beginning to blur [14, 22, 34, 59].

CA-MRSA can also be defined based on genetic markers, such as the presence of SCCmec type IV, V or VII, the presence of Panton-Valentine Leukocidin (PVL) and the association of several specific $S$. aureus lineages. PVL is a $S$. aureus-specific exotoxin that is part of the family of the bicomponent synergohymenotropic toxins, and kills leukocytes by creating pores in the cell membrane. PVL positive CA-MRSA strains are associated with skin infections and severe necrotizing pneumonia $[14,22,34]$. In general, between $40 \%$ and $90 \%$ of the MRSA isolates that carry SCCmec type IV are PVL positive. In MRSA isolates that harbor SCCmec type I, II or III, this percentage is less than $5 \%$. PVL has been heterogeneously distributed among isolates from different regions in the world [14]. Several studies described that PVL, together with SCCmec type IV or V and a specific genetic background is genetic marker for CA-MRSA. However as a consequence of the unequal distribution of PVL in isolates worldwide, PVL is not considered a genetic marker for CA-MRSA. Recent studies suggested that other factors also 
contribute to the virulence of CA-MRSA, such as the presence of porine forming toxins and the arginine catabolic genetic mobile element $[14,34]$. The secretion of phenol-soluble modulin by $S$. aureus is also associated with infections, because of recruitment, activation and lysis of neutrophils, resulting in destruction and failure of defence mechanisms of the body [14, 34]. Currently it is not clear whether these factors which give the $S$. aureus a selective advantage, play a crucial role in the possible increased virulence of CA-MRSA compared to HAMRSA. Further research is necessary to elucidate this question.

PFGE and MLST have shown that genetically CA-MRSA and HA-MRSA isolates are not related to each other. The larger clonal diversity of CA-MRSA compared to HA-MRSA suggests that more $S$. aureus lineages have the ability to become CAMRSA. Five major PVL positive CA-MRSA clones, i.e. ST1, ST8, ST30, ST59 and ST80 clones are disseminating worldwide. Several minor PVL-positive CA-MRSA clones have also been observed worldwide. Among them are the ST5, ST22, ST37, ST93, ST377, and ST766 clones. Of particular concern is the emergence of PVLpositive strains associated with ST5, since this lineage has a high capacity to spread resulting in an increased morbidity and mortality $[14,59]$. So far the origin of CA-MRSA is still unknown; it may be due to the acquisition of SCCmec by a MSSA or from a HA-MRSA strain. There are reports supporting the first hypothesis, however is has also been shown that a CA-MRSA and HA-MRSA clone may have a common ancestor [14].

\section{Hospital-acquired MRSA}

The last decades the rate of $S$. aureus infections has increased, resulting in an increased patient morbidity and mortality $[37,43]$. The mortality related to $S$. aureus bloodstream infections is between 15 and 60\% [7]. Surgical patients, ICU patients, patients with intravenous catheters and patients with other health care related diseases have a higher risk in developing $S$. aureus (bloodstream) infections. Moreover, MRSA colonised patients have a significant higher risk in developing bacteraemia than MSSA carriers and non-Staphylococcus aureus carriers [11, 54, 72]. Two recent meta-analyses showed that bloodstream infections due to MRSA are associated with a significant higher morbidity and mortality rate than MSSA infections [7]. The reason for this is not fully cleared, but the differences may be due to a higher virulence of some MRSA strains, the delay in microbiologically appropriate antibiotic treatment and the decreased effectiveness of glycopeptides compared to beta-lactam antibiotics, and the threat of glycopeptides resistance $[10,24]$. MRSA infections are associated with a significant longer hospital stay and higher treatment cost than infections due to MSSA or other pathogens $[11,42]$. Furthermore, because of the dissemination of 
MRSA in hospitals, MRSA infections may not only be replacing MSSA infections, but form an additional burden on the total number of $S$. aureus infections [24, 37].

\section{Cross-border health care}

There are five Euregions along the Dutch-German border. The Euregion MeuseRhine (EMR) is a region consisting of the Belgian provinces of Limburg and Liège, the German-speaking region of Belgium, the region Aachen in Germany, and the southern part of the Dutch province of Limburg. The region comprises about 30 hospitals in an area of $10,478 \mathrm{~km}^{2}$ with 3.9 million habitants. All Dutch-German Euregions have the same cross-border health care priorities: collaboration regarding patient transfer and admission between cross-border hospitals, crossborder use of rescue services, health care insurances companies, detection and prevention of infectious diseases like Sexual Transmitted Diseases and MRSA, and medico-technical developments between hospitals in the different countries [15, 22]. Cross-border patient mobility and free access to health care facilities within the European Union in general, and in the EMR in particular, are important issues for patients living in the Euregion. An important issue of concern related to crossborder health care is the dissemination of multi-resistant bacteria between the three countries. In Belgium, Germany, and the Netherlands the prevalence of MRSA isolated in hospitals differ considerably, i.e. $23.6 \%, 13.8 \%$, and $0.6 \%$ respectively [15]. Consequently, cross-border transfer of patients may have an important impact on the dissemination and prevalence of MRSA, in particular in cases where patients are transferred from countries with a relatively high prevalence to a country with a low prevalence [15].

In a recent cross-border study, MRSA isolates from hospitals in the EMR showed a greater genetic diversity in MRSA clones from Dutch hospitals, compared to clones from Belgian and German hospitals. Furthermore it was the first time that the New-York/Japan clone (ST5-MRSA-II) and the Paediatric clone (ST5-MRSA-IV) were detected in the Netherlands, which suggests a cross-border transfer of MRSA clones from abroad into the Netherlands [15, 48]. A study of MRSA clones isolated from patients in the University Hospital Maastricht, showed that until 2005 the ST5-MRSA-IV clone was predominant, with two spa types, t002 and t447, differing by just one repeat. The development of two spa types in one MRSA clone may be either attributed to a fitness adaption or to the introduction of a new clone. From 2005 on the clonal diversity were more divers. Certain newly introduced ST5-MRSA-IV and ST228-MRSA-I clones were cultured from patients and HCWs working in Belgian hospitals [48]. Recently, Grundmann et al. showed that MRSA clones in Europe occur predominantly in geographical 
clusters, which suggest that MRSA circulate through regional health care networks. Their conclusion was that infection control measures aimed at interrupting the spread of MRSA within and between health care institutions might be ultimately successful [25]. The clonal diversity stressed the importance in the potential threat of cross-border spread of MRSA from Belgian and German to Dutch hospitals. Harmonisation of infection control protocols in the hospitals in the three countries of the Euregions is therefore essential.

\section{Contact with livestock}

Recently, a MRSA clone has been observed among pigs, pig farmers and their family members in the Netherlands which was not related to the traditional known CA-MRSA or HA-MRSA clones. The prevalence of this clone in pig farmers was much higher than the MRSA prevalence in the general Dutch population [68]. These isolates were not typeable by PFGE with restriction endonuclease Smal due to the presence of a new DNA methylation enzyme. Further analysis showed that this MRSA clone belongs to ST398, and that the majority were either spa type t011, t034, or t108 $[14,68]$. Colonisation of several livestock and companion animals with this clone has been reported. Contact with pigs and cattle were an independent risk factor for MRSA carriage [36]. Therefore, infection control measures for livestock farmers, their relatives, and professionals having contact with living animals, such as veterinarians, when admitted to a hospital were implemented [64].

\section{Active surveillance and containment of MRSA}

After the appearance of MRSA in hospitals it has become clear that if no infection control program is implemented, MRSA will spread and colonise new patients and HCWs [3]. Transmission between patients without any infection control precautions is 16 times higher compared to patients in contact isolation [62]. Transmission appears eight times more often between patient than between HCWs and patients [6]. In the ICU the transmission is higher than in a regular ward, due to more patient-HCW contacts, higher use of antibiotics and the vulnerability of the patient population [7].

Several studies demonstrated the effect of pro-active screening and isolation of (high risk) patients admitted to the hospital to decrease the burden of MRSA colonised patients [24]. There are several strategies for screening patients. With the universal screening all patients admitted to the hospital are screened, regardless of their MRSA risk profile. A mathematical model has suggested that this approach is the most effective approach in the containment of MRSA, but 
also the most costly for the hospital [3, 28, 59]. The selective screening of patients is the most widely used policy in hospitals for reasons of cost and logistics. This screening includes that patients on admittance will be screened for the presence of risk factors, and then categorised in low or high risk. Patients admitted to a foreign hospital for at least 24 hours in the preceding six months and with an additional risk factor for MRSA colonisation, such as recent surgery, skin defects, intubation for mechanical ventilation, are considered as MRSA high risk patients. In addition, patients with a history of MRSA colonisation or infection in the previous six months are also considered as high risk. Patients admitted to a foreign hospital for at least 24 hours in the preceding six months and without any other risk factors for MRSA colonisation at the day of admission are considered as MRSA low-risk patients. This approach is however not easily implemented, because of limited compliance of the nursing staff to assess the risk profile based on the medical history [59]. Another drawback with this policy is that individuals with possible CA-MRSA colonisation, thus without any MRSA risk factors, may be missed at admission. Finally, a third approach is to screen all patients from departments at high risk for MRSA transmission and invasive infections, such as patients from intensive care, dialysis, and cardiothoracic surgery units [61].

MRSA colonised and high risk patients are nursed in isolation and decolonised, according to the local and/or national policy, until MRSA is eradicated. Ideally these patients are nursed in single low pressure rooms, but this is only possible in a low prevalence setting because of limited availability of these rooms. In high prevalence hospitals patients are put in single rooms or are cohorted. Besides standard precautions, HCWs have to take additional precautions such as wearing gloves, masks and gowns when entering the patients' room. Other measures that are implemented in the containment of MRSA are the optimal use of antibiotics, taking screening cultures, decolonisation of the MRSA positive patient with local application of mupirocin use of disinfecting soap and additional cleaning and disinfection of the room.

Since the introduction of the Search and Destroy (S\&D) policy in the Netherlands, MRSA carriers are pro-active identified. As carriers are early identified and isolated, the risk of transmission to other patients and HCWs decreased. Because of this policy, in the Netherlands as well as in the Nordic countries MRSA colonisation in hospitalised patients is below $1 \%[6,66,67]$.

\section{Cost of the MRSA containment}

Many studies have been published dealing with the financial cost of MRSA infections. As the methods used for cost calculation were highly variable, the 
studies were difficult to compare [24]. The methods for calculations of the costs used did not always reflect the actual cost of MRSA infections. In addition, the complex area of cost of morbidity after discharge and mortality was not taking into account. After discharge, MRSA positive patients are usually confronted with extended convalescence including attempts to decolonisation, drug exposure, psychological problems and anxiety if colonisation continues. After readmission in the hospital, they are again nursed in isolation, exposing them to a lot of stress. Carriage of MRSA is a risk factor for developing a new infection with increased morbidity and mortality. The major cost for the patient is death, but few data exist on the attributable risk on mortality due to MRSA [24]. The cost for the hospital is also substantial, and although MRSA colonisation itself is negligible, carriers are a potential hazard to others. However, the additional cost of a MRSA infection may vary considerably depending on the site of the infection and, whether the cost are compared to the cost for patients with a MSSA infection or to those without any infection.

Currently there is still much debate on the effectiveness of the precautions. On one hand it is clear that the implementation of any policy is at increased cost for hospitals, due to screening of patients, isolating of MRSA positive or high risk patients, increased use of disposables and the need for extra HCWs. On the other hand the cost related to MRSA infections may exceed the cost of MRSA containment. Therefore, all efforts to decrease the burden of MRSA may be costeffective. MRSA containment cost vary due to the policy of the hospitals, but far more important is the cost for the increased length of stay due to a MRSA infection, which itself may vary considerably according to the site of infection [24].

There have been publications on the cost of different infection control measures and of the containment of MRSA in certain wards of the hospital [24]. The cost of a MRSA containment program in an ICU setting was between $\$ 340$ and $\$ 1,480$ per patient; however the mean cost attributable to MRSA infections in ICU patients was $\$ 9,275$. A reduction of the transmission with $14 \%$ showed that this containment strategy was cost beneficial, even with a carriage rate up to $7 \%$ [5]. In a comparison between two neonatal units with MRSA outbreaks, the additional excess cost of nosocomial MRSA bloodstream infections in one unit without any infection control program was much higher, compared with the total cost when infection control measures were taken in the other unit [32]. MRSA screening of potential MRSA carriers on admission during a 19 month period prevented 35.2 MRSA infections, thereby saving $€ 200,782.73$ in revenues, while the screening cost was $€ 26,241.51$. A sensitivity analysis indicated that the 
screening strategy was still cost-effective if $<13.7 \%$ of all patients met the screening criteria [70].

So far, there are few publications on the cost of the Search and Destroy policy in low prevalence countries, as the Netherlands [46, 66, 69]. Vriens et al. estimated the yearly cost of the search and destroy policy in a Dutch university hospital to be $€ 280,000$. Furthermore not implementing such a policy was estimated to increase the MRSA prevalence gradually to $50 \%$ with an additional cost of $€ 520,000$ a year for antibiotics [69]. A recent publication by van Rijen et al. estimated the cost of the S\&D policy in a tertiary hospital in the Netherlands at $€ 215,559$ a year. In addition, the study estimated to have prevented an additional 36 cases of MRSA bacteraemia [66]. Nulens et al. compared the cost of pro-active screening and isolation of patients with MRSA risk factors, with the actual treatment cost of $S$. aureus infections. The total amount yearly spent in the University Hospital Maastricht for MRSA containment and treatment of $S$. aureus infections was $€ 1,381,200$ and $€ 2,738,128$ respectively. Furthermore, it was shown that this preventive strategy was still cost-effective if the MRSA prevalence rate was less than $8 \%$ [46].

It is obvious that whatever approach is implemented to detect MRSA carriers, a considerable amount of money is spent to prevent the transmission and dissemination of MRSA in hospitals. However, without any infection control, MRSA will disseminate among patients and HCWs in the hospital with an additional increase of MRSA infections and an increased morbidity and mortality [3]. On the other hand, a cost-effective infection control strategy should also be considered even in hospitals with a high MRSA prevalence. Using mathematical models, Bootsma et al. showed that with pro-active actions MRSA could be eradicated from the hospital within six years [3]. Therefore, well designed studies are needed to show the effectiveness of MRSA containment, and infection control [61].

Whatever pro-active approach is used in hospitals, several conditions must be fulfilled in advance. As MRSA transmission occur in spite of compliance of HCWs with standard precautions, active surveillance should be adjusted to the patient isolation capacity, the effectiveness in controlling spread and prevention of infections [61]. 


\section{References}

1. Aldeyab MA, Kearney MP, Hughes CM, Scott MG, Tunney MM, Gilpin DF, Devine MJ, Watson JD, Gardiner A, Funston C, Savage K, McElnay JC. Can the use of a rapid polymerase chain screening method decrease the incidence of nosocomial meticillin-resistant Staphylococcus aureus? J Hosp Infect. 2009;71: 22-28.

2. Bode LGM, Kluytmans JAJW, Wertheim HFL, Bogaers D, Vandenbroucke-Grauls CMJE, Roosendaal R, Troelstra A, Box ATA, Voss A, van der Tweel I, van Belkum A, Verbrugh HA, Vos MC. Preventing Surgical-Site Infections in Nasal Carriers of Staphylococcus aureus. N Engl J Med. 2010;362:9-17.

3. Bootsma MC, Diekmann O, Bonten MJ. Controlling methicillin-resistant Staphylococcus aureus: quantifying the effects of interventions and rapid diagnostic testing. Proc Natl Acad Sci USA. 2006;103:5620-5625.

4. Brown DFJ, Edwards DI, Hawkey PM, Morrison D, Ridgway GL, Towner KJ, Wren MWD on behalf of the Joint Working Party of the British Society for Antimicrobial Chemotherapy, Hospital Infection Society and Infection Control Nurses Association. Guidelines for the laboratory diagnosis and susceptibility testing of methicillin-resistant Staphylococcus aureus (MRSA). J Antimicrob Chemother. 2005;56:1000-1018.

5. Chaix C, Durand-Zaleski I, Alberti C, Brun-Buisson C. Control of endemic methicillin-resistant Staphylococcus aureus: a cost-benefit analysis in an intensive care unit. JAMA. 1999;282:17451751.

6. Cooper BS, Medley GF, Stone SP, Kibbler CC, Cookson BD, Roberts JA, Duckworth G, Lai R, Ebrahim S. Methicillin-resistant Staphylococcus aureus in hospitals and the community: stealth dynamics and control catastrophes. Proc Natl Acad Sci USA. 2004;101:10223-10228.

7. Cosgrove SE, Sakoulas G, Perencevich EN, Schwaber MJ, Karchmer AW, Carmeli Y. Comparison of mortality associated with methicillin-resistant and methicillin-susceptible Staphylococcus aureus bacteremia: a meta-analysis. Clin Infect Dis. 2003;36:53-58.

8. Crisóstomo MI, The evolution of methicillin resistance in Staphylococcus aureus: similarity of genetic backgrounds in historically early methicillin-susceptible and -resistant isolates and contemporary epidemic clones. Proc Natl Acad Sci USA. 2001;98:9865-9870.

9. Cunningham R, Jenks P, Nortwood J, Wallis M, Ferguson S, Hunt S. Effect on MRSA transmission of rapid PCR testing of patients admitted to critical care. J Hosp Infect. 2007;65:24-28.

10. Dancer SJ. The effect of antibiotics on methicillin-resistant Staphylococcus aureus. J Antimicrob Chemother. 2008;61:246-253.

11. Davis KA, Stewart JJ, Crouch HK, Florez CE, Hospenthal DR. Methicillin-resistant Staphylococcus aureus (MRSA) nares colonisation at hospital admission and its effect on subsequent MRSA infection. Clin Infect Dis. 2004;39:776-782.

12. Deurenberg RH, Vink C, Oudhuis GJ, Mooij JE, Driessen C, Coppens G, Craeghs J, De Brauwer E, Lemmen S, Wagenvoort H, Friedrich AW, Scheres J, Stobberingh EE. Different clonal complexes of methicillin-resistant Staphylococcus aureus are disseminated in the Euregio Meuse-Rhine Region. Antimicrob Agents Chemother. 2005;49:4263-4271

13. Deurenberg RH, Vink C, Kalenic S, Friedrich AW, Bruggeman CA, Stobberingh EE. The molecular eveolution of methicillin-resistant Staphylococcus aureus. Clin Microbiol Infect. 2006;13:222-235. 
14. Deurenberg RH, Stobberingh EE. The evolution of Staphylococcus aureus. Infect Genet Evol. 2008;8:747-763.

15. Deurenberg RH, Nulens E, Valvatne H, Sebastian S, Driessen C, Craeghs J, De Brauwer E, Heising B, Kraat YJ, Riebe J, Stals FS, Trienekens TA, Scheres J, Friedrich AW, van Tiel FH, Beisser PS, Stobberingh EE. Cross-border dissemination of methicillin-resistant Staphylococcus aureus, Euregio Meuse-Rhine region. Emerg Infect Dis. 2009;15:727-734.

16. Donnio PY, Février F, Bifani P, Dehem M, Kervégant C, Wilhelm N, Gautier-Lerestif AL, Lafforgue $\mathrm{N}$, Cormier M, MR-MSSA Study Group of the Collège de Bactériologie-Virologie-Hygiène des Hôpitaux de France, Le Coustumier A. Molecular and epidemiological evidence for spread of multi-resistant methicillin-susceptible Staphylococcus aureus strains in hospitals. Antimicrob Agents Chemother. 2007;51:4342-4350.

17. Enright M, Robinson DA, Randle G, Feil EJ, Grundmann H, Spratt BG. The evolutionary history of methicillin-resistant Staphylococcus aureus (MRSA). Proc Natl Acad Sci USA. 2002;99:7687-7692.

18. Faria NA, Carrico JA, Oliveira DC, Ramirez M, de Lencastre H. Analysis of typing methods for epidemiological surveillance of both methicillin-resistant and methicillin-susceptible Staphylococcus aureus strains. J Clin Microbiol. 2008;46:136-144.

19. Francois $P$, Bento M, Renzi G, Harbarth S, Pittet D, Schrenzel J. Evaluation of three molecular assays for rapid identification of methicillin-resistant Staphylococcus aureus. J Clin Microbiol. 2007;45:2011-2013.

20. Frénay HM, Bunschoten AE, Schouls LM, van Leeuwen WJ, Vandenbroucke-Grauls CM , Verhoef J, Mooi FR. Molecular typing of methicillin-resistant Staphylococcus aureus on the basis of protein A gene polymorphism. Eur J Clin Microbiol Infect Dis. 1996;15:60-64.

21. French GL. Methods for screening for methicillin-resistant Staphylococcus aureus carriage. Clin Microbiol Infect. 2009;15(Suppl 7):10-16.

22. Friedrich AW, Daniels-Haardt I, Köck R, Verhoeven F, Mellmann A, Harmsen D, van Gemert-Pijnen JE, Becker K, Hendrix MGR. EUREGIO MRSA-net Twente/Münsterland-a Dutch-German crossborder network for the prevention and control of infections caused by methicillin-resistant Staphylococcus aureus. Eurosurveillance. 2008;13:1-5.

23. Gordon RJ, Lowy FD. Pathogenisis of methicillin-resistant Staphylococcus aureus infection. Clin Infect Dis. 2008;46:S350-359.

24. Gould IM. Costs of hospital-acquired methicillin-resistant Staphylococcus aureus (MRSA) and its control. 2006 Int J Antimicrob Agents. 2006;28:379-384.

25. Grundmann H, Aanensen DM, van den Wijngaard CC, Spratt BG, Harmsen D, Friedrich AW; European Staphylococcal Reference Laboratory Working Group. Geographic distribution of Staphylococcus aureus causing invasive infections in Europe: a molecular-epidemiological analysis. PLoS Med. 2010;7:e1000215.

26. Hallin M, Denis O, Deplano A, De Ryck R, Crèvecoeur S, Rottiers S, de Mendonça R, Struelens MJ. Evolutionary relationships between sporadic and epidemic strains of health care-associated methicillin-resistant Staphylococcus aureus. Clin Microbiol Infect. 2008;14:659-669.

27. Harmsen D, Claus H, Witte W, Rothgänger J, Claus H, Turnwald D, Vogel U. Typing of methicillinresistant Staphylococcus aureus in a university hospital setting by using novel software for spa repeat determination and database management. J Clin Microbiol. 2003;41:5442-5448. 
28. Heyneman B, Pinxten S, Lenez A, Nulens E, Gordts B. Universal Admission Screening as the next successful step in an MRSA containment strategy. $7^{\text {th }}$ International Conference of the Hospital Infection Society, Liverpool, United Kingdom, 10 - 13 oktober2010. .

29. Huletsky A, Giroux R, Rossbach V, Gagnon M, Vaillancourt M, Bernier M, Gagnon F, Truchon K, Bastien M, Picard FJ, van Belkum A, Ouellette M, Roy PH, Bergeron MG. New real-time PCR assay for rapid detection of methicillin-resistant Staphylococcus aureus directly from specimens containing a mixture of staphylococci. J Clin Microbiol. 2004;42:1875-84.

30. International Working Group on the Classification of Staphylococcal Cassette Chromosome Elements (IWG-SCC). Classification of Staphylococcal Cassette Chromosome mec (SCCmec): Guidelines for Reporting Novel SCCmec Elements. Antimicrob Agents Chemother. 2009;53:49614967.

31. Jeyaratnam D, Whitty CJM, Phillips K, Liu D, Orezzi C, Ajoku U, French GL. Impact of rapid screening tests on acquisition of meticillin resistant Staphylococcus aureus: cluster randomised cross-over trial. BMJ. 2008;336:927-30

32. Karchmer TB, Durbin LJ, Simonton BM, Farr BM. Cost-effectiveness of active surveillance cultures and contact/droplet precautions for control of methicillin-resistant Staphylococcus aureus. J Hosp Infect. 2002;51:126-132.

33. Kelley PG, Grabsch EA, Howden BP, Gao W, Grayson ML. Comparison of the Xpert methicillinresistant Staphylococcus aureus (MRSA) assay, BD GeneOhm ${ }^{\mathrm{TM}}$ MRSA assay, and culture for detection of nasal and cutaneous groin colonisation by MRSA. J Clin Microbiol. 2009;47:37693772 .

34. Kennedy AD, DeLeo FR. Epidemiology and Virulence of Community-Associated MRSA. Clinical Microbiology Newsletter. 2009;31:153-160.

35. Kim J. Understanding the evolution of methicillin-resistant Staphylococcus aureus. Clin Microbiol Newlett. 2009;31:17-23.

36. Köck R, Harlizius J, Bressan N, Laerberg R, Wieler LH, Witte W, Deurenberg RH, Voss A, Becker K, Friedrich AW. Prevalence and molecular characteristics of methicillin-resistant Staphylococcus aureus (MRSA) among pigs on German farms and import of livestock-related MRSA into hospitals. Eur J Clin Microbiol Infect Dis. 2009;28:1375-1382.

37. Laupland KB, Ross T, Gregson DB. Staphylococcus aureus Bloodstream infections: risk factors, outcomes, and the influences of methicillin resistance in Calgary, Canada, 2000-2006. J Infect Dis. 2008;198:336-343.

38. Laurent C, Bogaerts P, Schoevaerdts D, Denis O, Deplano A, Swine C, Struelens MJ, Glupczynski Y. Evaluation of the Xpert MRSA assay for rapid detection of methicillin-resistant Staphylococcus aureus from nares swabs of geriatric hospitalised patients and failure to detect a specific SCCmec type IV variant. Eur J Clin Microbiol Infect Dis. 2010;29:995-1002.

39. Lindstedt BA. Multiple-locus variable number tandem repeats analysis for genetic fingerprinting of pathogenic bacteria. Electrophoresis. 2005;26:2567-2582.

40. Malachowa N, Sabat A, Gniadkowski M, Krzyszton-Russjan J, Empel J, Miedzobrodski J, KososkaShick K, Appelbaum PC, Hryniewicz W. Comparison of multiple-locus-number tandem-repeat analysis with pulsed-field gel electrophoresis, spa typing, and multi-locus sequence typing for clonal characterisation of Staphylococcus aureus isolates. J Clin Microbiol. 2005;42:3095-3100. 
41. Malhotra-Kumar S, Haccuria K, Michiels M, leven M, Poyart C, Hryniewicz W, Goossens H; MOSAR WP2 Study Team. Current trends in rapid diagnostics for methicillin-resistant Staphylococcus aureus and glycopeptide-resistant enterococcus species. J Clin Microbiol. 2008;46:1577-1587.

42. Melzer M, Eykyn SJ, Grandsen WR, Chinn S. Is methicillin-resistant Staphylococcus aureus more virulent than methicillin-susceptible $S$. aureus? A comparative cohort study of British patients with nosocomial infection and bacteremia. Clin Infect Dis. 2003;37:1453-1460.

43. Naber CK. Future strategies for treating Staphylococcus aureus bloodstream infections. Clin Microbiol Infect. 2008;14(Suppl. 2):26-34.

44. Noto MJ, Kreiswirth BN, Monk AB, Arche GL. Gene acquisition at the insertion site for SCCmec, the genomic island conferring methicillin resistance in Staphylococcus aureus. J Bacteriol. 2008;190:1276-1283.

45. Nulens E, Gould I, MacKenzie F, Deplano A, Cookson B, Alp E, Bouza E, Voss A. Staphylococcus aureus carriage among participants at the 13th European Congress of Clinical Microbiology and Infectious Diseases. Eur J Clin Microbiol Infect Dis. 2005;24:145-148.

46. Nulens E, Broex E, Ament A, Deurenberg RH, Smeets E, Scheres J, van Tiel FH, Gordts B, Stobberingh EE. Cost of the meticillin-resistant Staphylococcus aureus search and destroy policy in a Dutch university hospital. J Hosp Infect. 2008;68:301-307.

47. Nulens E, Stobberingh EE, van Dessel H, Sebastian S, van Tiel FH, Beisser PS, Deurenberg RH. Molecular characterisation of Staphylococcus aureus bloodstream isolates collected in a Dutch University Hospital between 1999 and 2006. J Clin Microbiol. 2008;46:2438-2441.

48. Nulens E, Stobberingh EE, Smeets E, van Dessel H, Welling MA, Sebastian S, van Tiel FH, Beisser PS, Deurenberg RH. Genetic diversity of methicillin-resistant Staphylococcus aureus in a tertiary hospital in the Netherlands between 2002 and 2006. Eur J Clin Microbiol Infect Dis. 2009;28:631639.

49. Nulens E, Descheemaeker P, Deurenberg RH, Stobberingh EE, Gordts B. Contribution of two molecular assays as compared to selective culture for MRSA screening in a low MRSA prevalence population. Infection. 2010;38:98-101.

50. Ogston A. Micrococcus poisoning. J Anat. 1882;24-58.

51. Oliveira DC, Tomasz A, de Lencastre H. Secrets of success of a human pathogen : molecular evolution of pandemic clones of meticillin-resistant Staphylococcus aureus. Lancet Infect Dis. 2002;2:180-189.

52. Petersson AC, Olsson-Liljequist B, Miörner H, Haeggman S. Evaluating the usefulness of spa typing, in comparison with pulsed-field gel electrophoresis, for epidemiological typing of methicillin-resistant Staphylococcus aureus in a low-prevalence region in Sweden 2000-2004. Clin Microbiol Infect. 2010;16(5):456-462.

53. Pourcel C, Hormigos K, Onteniente L, Sakwinska O, Deurenberg RH, Vergnaud G. Improved multiple-locus variable-number tandem-repeat assay for Staphylococcus aureus genotyping, providing a highly informative technique together with strong phylogenetic value. J Clin Microbiol. 2009;47:3121-3128.

54. Pujol M, Peña C, Pallares R, Ariza J, Ayats J, Dominguez MA, Gudiol F. Nosocomial Staphylococcus aureus bacteremia among nasal carriers of methicillin-resistant and methicillin-susceptible strains. Am J Med. 1996;100:509-516.

55. Robinson DA, Enright MC. Evolutionary models of the emergence of methicillin-resistant Staphylococcus aureus. Antimicrob Agents Chemother. 2003;47:3926-3934. 
56. Rossney AS, Herra CM, Brennan GI, Morgan PM, O'Connell B. Evaluation of the Xpert methicillinresistant Staphylococcus aureus (MRSA) assay using the GeneXpert real-time PCR platform for rapid detection of MRSA from screening specimens. J Clin Microbiol. 2008;46:3285-3290.

57. Ruppitsch W, Indra A, Stöger A, Mayer B, Stadlbauer S, Wewalka G, Allerberger F. Classifying spa types in complexes improves interpretation of typing results for methicillin-resistant Staphylococcus aureus. J Clin Microbiol. 2006;44:2442-2448.

58. Salgado CD, Farr BM, Calfee DP. Community-acquired methicillin-resistant Staphylococcus aureus: a meta-analysis of prevalence and risk factors. Clin Infect Dis. 2003;36:131-139.

59. Schouls LM, Spalburg EC, van Luit M, Huijsdens XW, Pluister GN, van Santen-Verheuvel MG, van der Heide HG, Grundmann H, Heck ME, de Neeling AJ. Multiple-locus variable number tandem repeat analysis of Staphylococcus aureus: comparison with pulsed-field gel electrophoresis and spa-typing. PLoS One. 2009;4(4):e5082.

60. Snyder JW, Munier GK, Heckman SA, Camp P, Overman TL. Failure of the BD GeneOhm StaphSR assay for direct detection of methicillin-resistant and methicillin-susceptible Staphylococcus aureus isolates in positive blood cultures collected in the United States. J Clin Microbiol. 2009;47:3747-8.

61. Struelens MJ, Hawkey PM, French GL, Witte W, Tacconelli E. Laboratory tools and strategies for methicillin-resistant Staphylococcus aureus screening, surveillance and typing: state of the art and unmet needs. Clin Microbiol Infect. 2009;15:112-119.

62. Tacconelli E, De Angelis G, de Waure C, Cataldo MA, La Torre G, Cauda R. Rapid screening tests for meticillin-resistant Staphylococcus aureus at hospital admission: systematic review and metaanalysis. Lancet Infect Dis. 2009;9:546-554.

63. Tenover FC, Arbeit RD, Goering RV, Mickelsen PA, Murray BE, Persing DH, Swaminathan B. Interpreting chromosomal DNA restriction patterns produced by pulsed-field gel electrophoresis: criteria for bacterial strain typing. J Clin Microbiol. 1995;33:2233-2239.

64. Vandenbroucke-Grauls CMJE, Beaujean DJMA. Meticillineresistente Staphylococcus aureus bij veehouders. Ned Tijdschr Geneeskd. 2006;150:1710-1712.

65. van Belkum A. Tracing isolates of bacterial species by multi-locus variable number of tandem repeat analysis (MLVA). FEMS Immunol Med Microbiol. 2007;49:22-27.

66. van Rijen MML, Kluytmans JAJW. Costs and benefits of the search and Destroy policy in a Dutch hospital. Eur J Microbiol Infect Dis. 2009;28:1245-1252.

67. Vos MC, Behrendt MD, Melles DC, Mollema FP, de Groot W, Parlevliet G, Ott A, Horst-Kreft D, van Belkum A, Verbrugh HA. 5 years of experience implementing a methicillin-resistant Staphylococcus aureus search and destroy policy at the largest university medical center in the Netherlands. Infect Control Hosp Epidemiol. 2009;30:977-984.

68. Voss A, Loeffen F, Bakker J, Klaassen C, Wulf M. Methicillin-resistant Staphylococcus aureus in pig farming. Emerg Infect Dis. 2005;11:1965-1966.

69. Vriens M, Blok H, Fluit A, Troelstra A, Van Der Werken C, Verhoef J. Costs associated with a strict policy to eradicate methicillin-resistant Staphylococcus aureus in a Dutch University Medical Center: a 10-year survey. Eur J Clin Microbiol Infect Dis. 2002;21:782-786. 
70. Wassenberg MW, Kluytmans JA, Box AT, Bosboom RW, Buiting AG, van Elzakker EP, Melchers WJ, van Rijen MM, Thijsen SF, Troelstra A, Vandenbroucke-Grauls CM, Visser CE, Voss A, Wolffs PF, Wulf MW, van Zwet AA, de Wit GA, Bonten MJ. Rapid screening of methicillin-resistant Staphylococcus aureus using PCR and chromogenic agar: a prospective study to evaluate costs and effects. Clin Microbiol Infect. 2010. DOI: 10.1111/j.1469-0691.2010.03210.x

71. Wernitz MH, Keck S, Swidsinski S, Schulz S, Veit SK. Cost analysis of a hospital-wide selective screening programme for methicillin-resistant Staphylococcus aureus (MRSA) carriers in the context of diagnosis related groups (DRG) payment. Clin Microbiol Infect. 2005;11:466-471.

72. Wertheim HF, Vos MC, Ott A, van Belkum A, Voss A, Kluytmans JA, van Keulen PH, Vandenbroucke-Grauls CM, Meester $\mathrm{MH}$, Verburgh HA. Risk and outcome of nosocomial Staphylococcus aureus bacteraemia in nasal carriers versus non-carriers. Lancet. 2004;364:703705.

73. Wertheim HFL, Melles DC, Vos MC, van Leeuwen W, van Belkum A, Verburgh HA, Nouwen JL. The role of nasal carriage in Staphylococcus aureus infections. Lancet Infect Dis. 2005;5:751-763.

74. Wolk DM, Picton E, Johnson D, Davis T, Pancholi P, Ginocchio CC, Finegold S, Welch DF, de Boer M, Fuller D, Solomon MC, Rogers B, Mehta MS, Peterson LR. Multi-center evaluation of the Cepheid $\mathrm{Xpert}^{\mathrm{TM}}$ methicillin-resistant Staphylococcus aureus (MRSA) test as a rapid screening method for detection of MRSA in nares. J Clin Microbiol. 2009;47:758-764.

75. Wolk DM, Struelens MJ, Pancholi P, Davis T, Della-Latta P, Fuller D, Picton E, Dickenson R, Denis O, Johnson D, Chapin K. Rapid detection of Staphylococcus aureus and methicillin-resistant S. aureus (MRSA) in wound specimens and blood cultures: multi-center preclinical evaluation of the

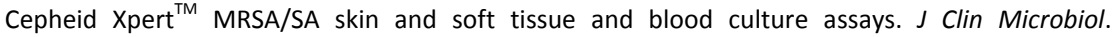
2009;47:823-826.

76. Zhang K, McClure JA, Elsayed S, Conly JM. Novel Staphylococcal Cassette Chromosome mec Type, Tentatively Designated Type VIII, Harboring Class A mec and Type $4 \mathrm{ccr}$ Gene Complexes in a Canadian Epidemic Strain of methicillin-resistant Staphylococcus aureus. Antimicrob Agents Chemother. 2009;53:531-540.

77. Zhang SX, Drews SJ, Tomassi J, Katz KC. Comparison of two versions of the IDI-MRSA assay using charcoal swabs for prospective nasal and non-nasal surveillance samples. J Clin Microbiol. 2007;45:2278-80. 


\section{Chapter 2: Outline of the thesis}

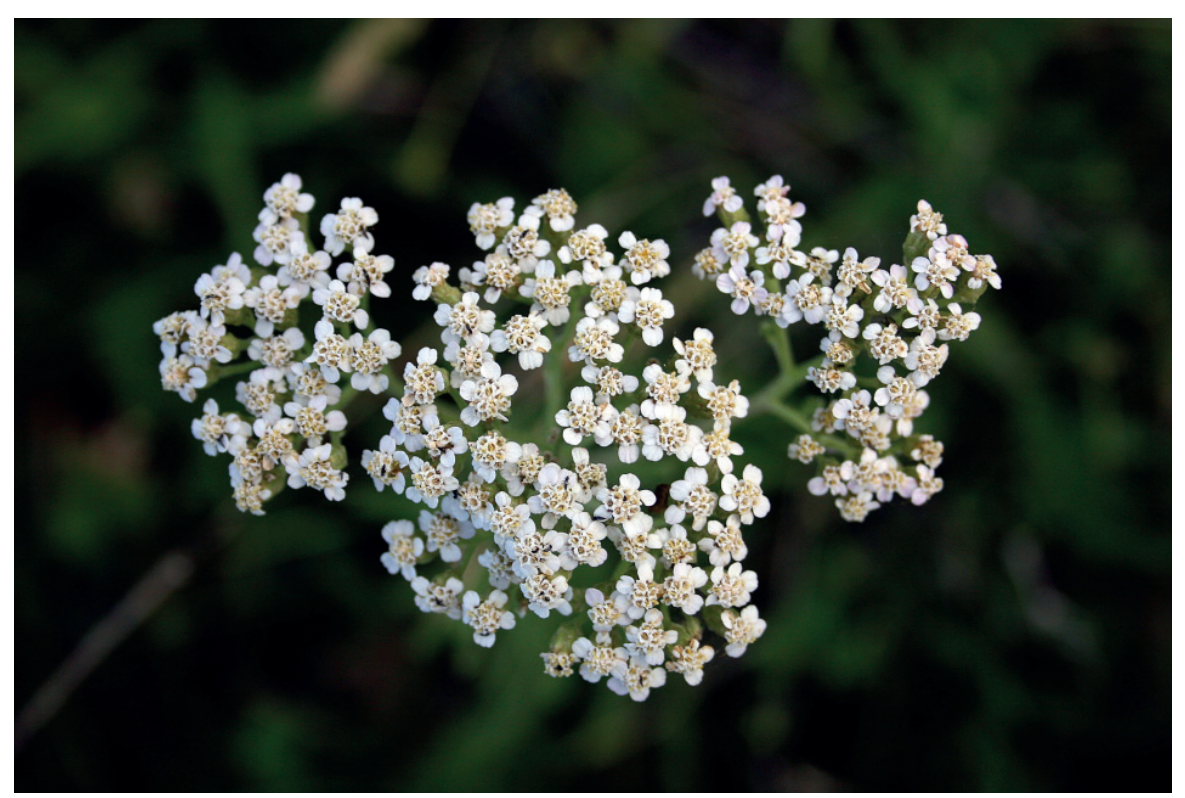

Achillea Mil, Brugge, 2009 


\section{Outline of the thesis}

Staphylococcus aureus is part of the indigenous flora of the skin and mucous membranes of healthy persons. Around $20 \%$ of healthy persons are permanently colonised with this micro-organism mainly in the nose, $50 \%$ is an intermittent carrier and in $30 \%$ the micro-organism is not part of the indigenous flora.

S. aureus is a frequent cause of community and hospital-acquired infections. In the community S. aureus is an important causative agent of skin infections such as folliculitis, furunculosis and impetigo. In the hospital setting, bloodstream infections and post-operative wound infections are frequently caused by $S$. aureus. In the majority of these infections the $S$. aureus is part of the indigenous flora of the patient as persistent carriers have a 3 to 6 times higher chance to get infected compared to non-carriers. Beside these so-called endogenous infections spread of certain epidemic clones can take place as well.

In the Netherlands, due to a stringent antibiotic and infection control policy, most S. aureus isolated in and outside the hospital are highly susceptible to betalactam antibiotics. This is in contrast to most other countries, the Scandinavian countries excepted. However, several risk factors such as increased antibiotic use and spread of resistant micro-organisms from abroad (i.e. cross-border spread) into the Netherlands contribute to a decrease in susceptibility to beta-lactam and other antimicrobial agents. This results in turn to an increase in prevalence of beta-lactam antibiotic resistant $S$. aureus, the so-called methicillin or multiresistant $S$. aureus. In addition to the resistance to methicillin and other agents, some of these isolates have the ability to easily spread between patients and between patients and health care workers in a hospital setting and even between health care centres. For hospitals located near the borders with Germany and Belgium spread of antibiotic resistant micro-organisms from abroad into the hospital is an additional challenge. Rapid identification of patients colonised or infected with such antibiotic resistant micro-organisms is important to control and to prevent the spread of these bugs.

The aim of this thesis was to determine the characteristics of the major MRSA and MSSA types in the Euregion Meuse-Rhine, the financial burden of the Search and Destroy policy and the usefulness of molecular methods to rapidly identify MRSA carriers.

Chapter 1 gives an overview of the literature on methicillin-susceptible and resistant $S$. aureus in terms of prevalence, risk factors in and outside the 
hospitals, the different typing methods currently used and the cost to contain the MRSA problem.

The genetic characteristics of MSSA strains isolated from patients admitted to the University Hospital in Maastricht with bloodstream infections between 1999 and 2006 are described in Chapter 3 (Molecular characterisation of Staphylococcus aureus bloodstream isolates collected in a Dutch university hospital between 1999 and 2006). Isolates were typed by real-time amplification and sequencing of the spa gene. In Chapter 4 (Genetic diversity of methicillin-resistant Staphylococcus aureus in a tertiary hospital in the Netherlands between 2002 and 2006), the major MRSA types in the University Hospital in Maastricht cultured between 2002 and 2006 were characterised using SCCmec typing and spa typing. All isolates were almost exclusively from surveillance cultures. In Chapter $\mathbf{5}$ (Cross-border dissemination of methicillin-resistant Staphylococcus aureus, Euregion Meuse-Rhine region), the emergence, the diversity and the transmission of MRSA in the different hospitals in the Euregion by several typing methods were investigated with $\mathrm{SCCmec}$ and spa typing. The cost of the Search and Destroy policy as used in the University Hospital Maastricht is discussed in Chapter 6 (Cost of the meticillin-resistant Staphylococcus aureus search and destroy policy in a Dutch university hospital). The cost of the containment of MRSA is compared with the treatment cost of patients with a S. aureus infection. In Chapter 7 (Contribution of two molecular assays as compared to selective culture for MRSA screening in a low MRSA prevalence population), the usefulness of molecular methods to rapid identify MRSA carriers in a low MRSA population is presented. 



\section{Part 2: GENETIC BACKGROUND AND IMPACT OF STAPHYLOCOCCUS AUREUS IN A CROSS-BORDER REGION}

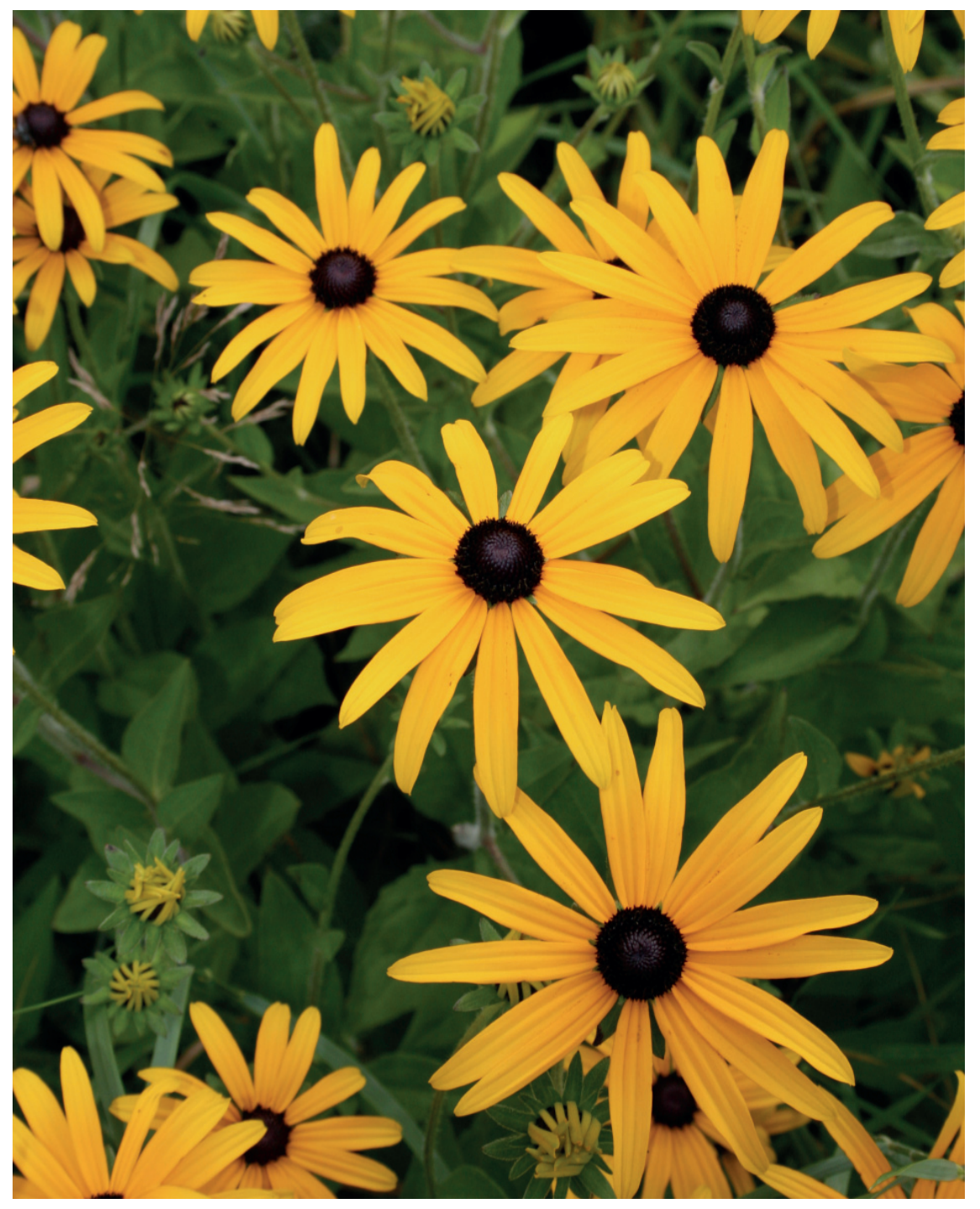

Thunbergia alata, Lanaken, 2008 



\section{Chapter 3: Molecular characterisation of Staphylococcus aureus bloodstream isolates collected in a Dutch University Hospital between 1999 and 2006}

E. Nulens, E.E. Stobberingh, H. van Dessel, S. Sebastian, F.H. van Tiel, P.S. Beisser, R.H. Deurenberg

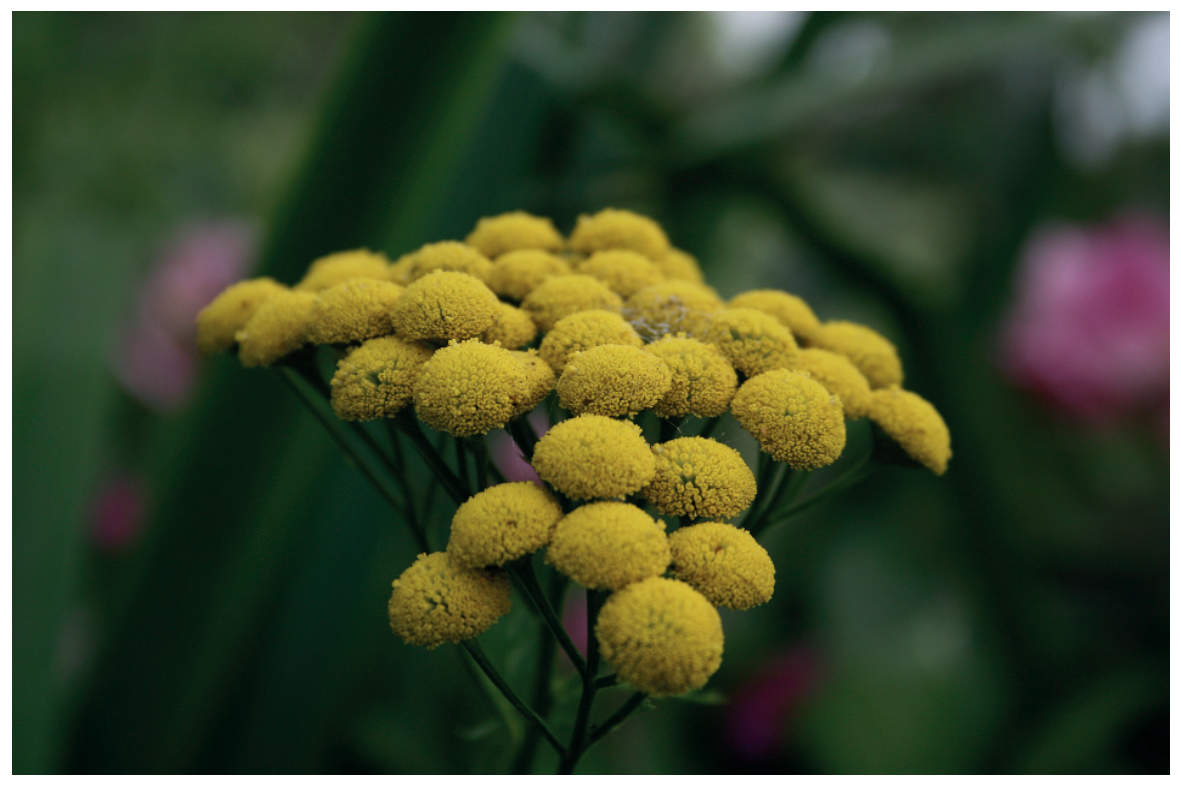

Santolina Chamaecyparissus, Brugge, 2009

Published in

Journal of Clinical Microbiology. 2008;46:2438-2441. 


\section{Abstract}

We observed that, between 1999 and 2006, up to 50\% of the methicillinsusceptible Staphylococcus aureus (MSSA) bloodstream isolates in our hospital had a genetic background common to endemic methicillin-resistant $S$. aureus clones (clonal complex 5 [CC5], CC8, CC22, CC30, and CC45). Furthermore, several successful MSSA lineages, such as CC7 and CC15, were observed. 
Methicillin-resistant Staphylococcus aureus (MRSA) can cause a wide variety of infectious diseases [15]. MRSA has a clonal population structure, and five major clonal complexes (CCs), i.e., CC5, CC8, CC22, CC30, and CC45, as determined with multi-locus sequence typing (MLST), have been observed [4]. In MRSA, $\beta$-lactam antibiotic resistance is mediated by the staphylococcal cassette chromosome mec

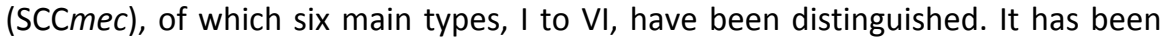
suggested that MRSA originated through the transfer of SCCmec from MRSA into methicillin-susceptible $S$. aureus (MSSA) and that the genetic background determines the stability of the new MRSA clone $[9,13]$.

Since no information exists about the MSSA population structure in Dutch hospitals and the relation to MRSA lineages, the genetic background of MSSA in the Maastricht University Hospital was investigated with spa typing.

Between 1999 and 2006, 501 MSSA bloodstream isolates were cultured from individual patients in the Maastricht University Hospital, a tertiary 715-bed university hospital. For each year, the first 25 MSSA isolates, corresponding to ca. $40 \%$ of the isolates, were analysed. Several isolates were not viable when cultured from the stocks and were excluded from the study.

Real-time amplification of the spa locus was performed with the primers spa$1113 \mathrm{~F}$ and spa-1514R [1]. The reaction conditions included $0.5 \mu \mathrm{M}$ spa-1113F, $0.25 \mu \mathrm{M}$ spa-1514R, 1x iQ Sybr green Supermix (Bio-Rad Laboratories, The Netherlands), and $5 \mu$ of a suspension of $1.5 \times 10^{8} \mathrm{CFU} / \mathrm{ml}$ of the isolate in a total volume of $25 \mu \mathrm{l}$. The amplification was performed on a Bio-Rad MyiQ singlecolour real-time PCR detection system, using the following program: $4 \mathrm{~min}$ at $95^{\circ} \mathrm{C}$ and $30 \mathrm{~s}$ at $65^{\circ} \mathrm{C}$, followed by 35 cycles of $30 \mathrm{~s}$ at $65^{\circ} \mathrm{C}, 15 \mathrm{~s}$ at $95^{\circ} \mathrm{C}$, and $30 \mathrm{~s}$ at $65^{\circ} \mathrm{C}$. Sequencing of the spa locus was performed as described previously [10]. The spa types were assigned through the Ridom SpaServer (http://spaserver. ridom.de) and clustered into spa-CCs using the algorithm based upon repeat pattern (BURP) with Ridom StaphType 1.4 using the default settings [5, 22]. Since it has been shown that spa typing/BURP results are in agreement with results obtained by MLST $[20,22]$, the associated MLST CCs were allocated through the SpaServer.

Ninety-three spa types were observed among the MSSA isolates. These spa types were clustered into 13 spa-CCs, seven singletons, and four spa types that were excluded from the analysis, because the spa locus was less than five spa repeats in length [17] (Table 1). The most common spa types were t091 (12.2\%), t084 (6.7\%), t002 (4.4\%), t008 (3.9\%), t127 (3.9\%), t012 (3.3\%), and t015 (3.3\%). Each 
of the remaining 86 spa types (62.3\% of the isolates) accounted for between 2.2 and $0.6 \%$ each.

The main spa-CC was spa-CC012, which harbored $43 \%$ of the MSSA isolates (Table 1). This spa-CC consisted of various MLST CCs. It consisted mainly of MSSA associated with CC7 ( $n=23$; founder t091), CC15 ( $n=14$; founder t084), and CC30 ( $n=26$; founder t012) (Figure 1). Additional spa-CCs included spa-CC008 (9\%), spaCC002 (9\%), spa-CC015 (6\%), and spa-CC127 (6\%), associated with CC8, CC5, CC45, and CC1, respectively. The remaining spa-CCs accounted for $3 \%$ or less of the isolates (Table 1). Four of the seven isolates that were excluded from BURP analyses were associated with CC45. No spa-CC was replaced with another spaCC during the study period (Table 2 ).

TABLE 1. Distribution of spa types and spa-CCs among MSSA isolates

\begin{tabular}{|c|c|c|c|c|}
\hline spa-CC type & $\begin{array}{l}\text { No. (\%) } \\
\text { f strains }\end{array}$ & $\begin{array}{c}\text { No. (\%) } \\
\text { of spa } \\
\text { types }\end{array}$ & spa types & $\begin{array}{l}\text { Associated } \\
\qquad \mathrm{CC}(\mathrm{s})^{\mathrm{a}}\end{array}$ \\
\hline spa-CC012 & $78(43)$ & $32(34)$ & $\begin{array}{l}\text { t012, t018, t019, t021, t084, t091, t189, t209, } \\
\text { t228, t230, t267, t275, t342, t547, t786, t803, } \\
\text { t975, t1642, t1867, t1870, t1871, t1872, t1874, } \\
\text { t1875, t1877, t1899, t1901, t1902, t1906, t1932, } \\
\text { t2036, t2072 }\end{array}$ & $\begin{array}{l}7,15,30, \\
45\end{array}$ \\
\hline spa-CC008 & $16(9)$ & $9(10)$ & $\begin{array}{l}\text { t008, t024, t064, t068, t400, t723, t818, t1905, } \\
\text { t2034 }\end{array}$ & 8 \\
\hline spa-CCOO2 & $16(9)$ & $8(9)$ & t002, t010, t045, t179, t306, t447, t548, t1305 & 5 \\
\hline spa-CC150/078 & $6(3)$ & $5(5)$ & t056, t078, t150, t1312, t1671 & 25,101 \\
\hline spa-CCO05 & $6(3)$ & $5(5)$ & t005, t309, t1869, t1903, t2037 & 22 \\
\hline spa-CC015 & $10(6)$ & $5(5)$ & t015, t040, t050, t1904, t2035 & 45 \\
\hline spa-CC127 & $10(6)$ & $4(4)$ & $\mathrm{t} 127, \mathrm{t} 177, \mathrm{t} 273, \mathrm{t} 1868$ & 1 \\
\hline spa-CC159 & $4(2)$ & $3(3)$ & $\mathrm{t} 159, \mathrm{t} 171, \mathrm{t} 645$ & 51 \\
\hline spa-CC240 & $4(2)$ & $3(3)$ & t136, t240, t1873 & \\
\hline No founder 1 & $5(3)$ & $2(2)$ & t160, t771 & 12 \\
\hline No founder 2 & $2(1)$ & $2(2)$ & $\mathrm{t} 148, \mathrm{t} 1348$ & \\
\hline No founder 3 & $5(3)$ & $2(2)$ & $\mathrm{t} 100, \mathrm{t} 193$ & 9 \\
\hline No founder 4 & $2(1)$ & $2(2)$ & t1907, t2040 & \\
\hline Singletons & $9(5)$ & $7(8)$ & t216, t257, t314, t587, t937, t1878, t1900 & 51,59 \\
\hline Excluded $^{b}$ & $7(4)$ & $4(4)$ & t026, t390, t605, t693 & 45 \\
\hline Total & $180(100)$ & $93(100)$ & & \\
\hline
\end{tabular}


TABLE 2. Distribution of $s p a-C C s$ in time (1999 to 2006)

\begin{tabular}{|c|c|c|c|c|c|c|c|c|c|}
\hline \multirow[b]{2}{*}{ spa-CC type } & \multicolumn{7}{|c|}{ No. of MSSA isolates by year } & \multirow[b]{2}{*}{2006} & \multirow[b]{2}{*}{ Total } \\
\hline & 1999 & 2000 & 2001 & 2002 & 2003 & 2004 & 2005 & & \\
\hline spa-CC012 & 9 & 10 & 8 & 9 & 11 & 8 & 8 & 15 & 78 \\
\hline spa-CC008 & 1 & 3 & 2 & 2 & 1 & 1 & & 6 & 16 \\
\hline spa-CC002 & 3 & 2 & 1 & 3 & 2 & & 2 & 2 & 15 \\
\hline spa-CC150/078 & & 2 & 1 & 1 & & 2 & & & 6 \\
\hline spa-CCO05 & & 1 & 1 & 1 & 1 & & 2 & & 6 \\
\hline spa-CC015 & & & 1 & & 3 & 4 & & 2 & 10 \\
\hline spa-CC127 & 1 & 2 & 3 & 2 & 1 & & 1 & & 10 \\
\hline spa-CC159 & & & 2 & & 1 & & 1 & & 4 \\
\hline spa-CC240 & & & 1 & 1 & 1 & & & 1 & 4 \\
\hline No founder 1 & 1 & & 1 & 1 & & 1 & 1 & & 5 \\
\hline No founder 2 & & & & & & & & 2 & 2 \\
\hline No founder 3 & 2 & 1 & & & & 1 & 1 & & 5 \\
\hline No founder 4 & & & & & 1 & 1 & & & 2 \\
\hline Singletons & 1 & 1 & & 3 & 1 & 1 & 1 & 1 & 9 \\
\hline Excluded $^{\mathrm{a}}$ & & 1 & 1 & & 2 & 2 & & 1 & 7 \\
\hline Total & 18 & 23 & 22 & 23 & 25 & 21 & 17 & 30 & 180 \\
\hline
\end{tabular}

${ }^{\mathrm{a}}$ That is, spa types smaller than five spa repeats.

Up to $50 \%$ of the MSSA had a genetic background observed in epidemic hospitalassociated MRSA clones, i.e., CC5, CC8, CC22, CC30, or CC45 [4], and communityassociated (CA) MRSA clones, i.e., CC1, CC8, CC30, or CC59 [23]. Although we took a random sample of the MSSA isolates available, our results are comparable to a study in Belgium, in which $45 \%$ of the MSSA isolates had a genetic background common to MRSA clones [8]. MSSA associated with CC1, CC5, CC8, CC30, and CC45 have been described previously in Brazil, Germany, among Danish isolates from the 1960s and 1970s, and in the Dutch and English community $[6,7,18,19,24,25]$. CC59 is a common CA-MRSA lineage in Asian countries, such as Singapore and Taiwan, but has also recently been observed in The Netherlands [3, 11, 12, 23]. The observation that MSSA with genetic backgrounds common to MRSA were found might suggest that these MSSA isolates could be a recipient for $\mathrm{SCCmec}$, since this element is suggested to be mobile [9].

Several MSSA lineages were found that were not associated with MRSA, such as CC7, CC9, CC12, CC15, CC25, CC51, and CC101. The observation that more MSSA CCs were found compared to MRSA CCs suggests that MSSA is more heterogeneous. Similar results have been observed in Belgium, Brazil, England, 
Germany, and Portugal [2, 7, 8, 14, 25]. Recently, MSSA associated with CC7, CC9, CC12, CC15, CC25, CC51, and CC101 have been observed in Belgium and in the English community, although no MRSA strains associated with these lineages were found $[7,8]$. Similarly, in Portugal, MSSA strains associated with CC9, CC12, $\mathrm{CC} 15, \mathrm{CC} 51$, and $\mathrm{CC} 25$ have been found in the community and in hospitals, whereas no MRSA strains from these CCs were observed [2]. The CC51 genetic background has been found among MSSA in Denmark from the 1960s and in the Dutch community between 1997 and 2002 [6, 16]. The observation that 50\% of the MSSA in the present study had a genetic background uncommon to MRSA clones may suggest that these genetic backgrounds do not provide a genomic environment necessary for the stable integration of SCCmec, as has been shown in a previous study [13].

Previous studies have shown a good concordance between spa typing/BURP and MLST [20, 22]. The observation that spa-CC012 was heterogeneous (Figure 1a) could be explained by the fact that large chromosomal replacements, including the spa locus, between different $S$. aureus lineages have occurred, as has been shown previously between CC8 and $30[18,22]$, or could be due to related spa repeat successions in different MSSA lineages, possibly caused by recombination in the spa locus (Figure 1b) [21]. A spa-CC containing CC7 and CC15 has been described previously [20]. 


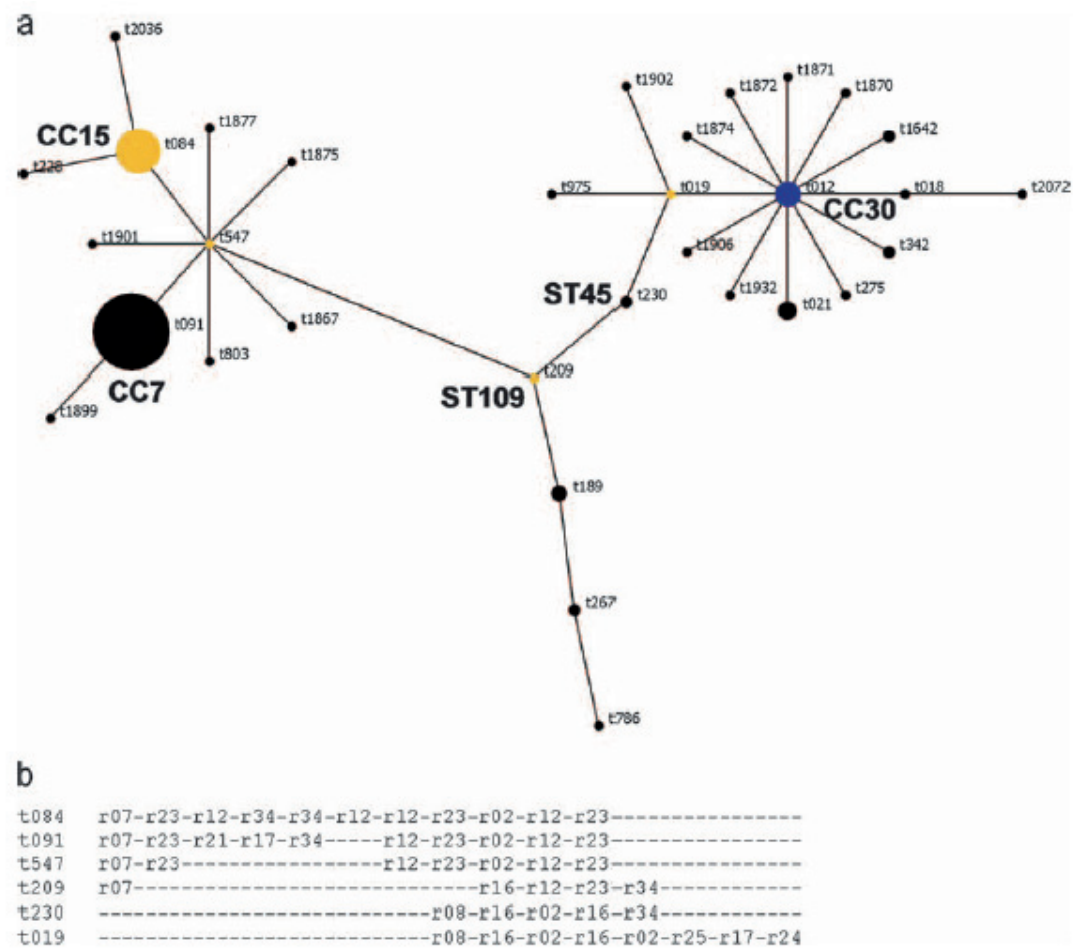

FIGURE 1. (a) Heterogeneous clonal structure of spa-CC012. At the right, spa type t012 is the founder of CC30, and at the left, spa type t084 is the founder of CC15 and spa type t091 is the founder of CC7. (b) Related spa repeat successions of the spa types of spa-CC012

\section{Acknowledgements}

We thank Hajo Grundmann from the National Institute of Public Health and the Environment in The Netherlands for critical reading of the manuscript. 


\section{References}

1. Aires-de-Sousa $M$, Boye $K$, de Lencastre $H$, Deplano A, Enright $M C$, Etienne J, Friedrich A, Harmsen D, Holmes A, Huijsdens XW, Kearns AM, Mellmann A, Meugnier $H$, Rasheed JK, Spalburg E, Strommenger B, Struelens MJ, Tenover FC, Thomas J, Vogel U, Westh H, Xu J, Witte W. High interlaboratory reproducibility of DNA sequence-based typing of bacteria in a multi-center study. J Clin Microbiol. 2006;44:619-621.

2. Aires de Sousa M, Conceicao T, Simas C, de Lencastre H. Comparison of genetic backgrounds of methicillin-resistant and susceptible Staphylococcus aureus isolates from Portuguese hospitals and the community. J Clin Microbiol. 2005;43:5150-5157.

3. Boyle-Vavra S, Ereshefsky B, Wang CC, Daum RS. Successful multi-resistant community-associated methicillin-resistant Staphylococcus aureus lineage from Taipei, Taiwan, that carries either the novel staphylococcal chromosome cassette mec (SCCmec) type $\mathrm{V}_{\mathrm{T}}$ or SCCmec type IV. J Clin Microbiol. 2005;43:4719-4730.

4. Deurenberg RH, Vink C, Kalenic S, Friedrich AW, Bruggeman CA, Stobberingh EE. The molecular evolution of methicillin-resistant Staphylococcus aureus. Clin Microbiol Infect. 2007;13:222-235.

5. Friedrich AW, Witte W, Harmsen D, de Lencastre H, Hryniewicz W, Scheres J, Westh H. SeqNet.org: a European laboratory network for sequence-based typing of microbial pathogens. Euro Surveill. 2006;11: E060112-E060114.

6. Gomes AR, Westh $\mathrm{H}$, de Lencastre H. Origins and evolution of methicillin-resistant Staphylococcus aureus clonal lineages. Antimicrob Agents Chemother. 2006;50:3237-3244.

7. Grundmann H, Hori S, Enright MC, Webster C, Tami A, Feil EJ, Pitt T. Determining the genetic structure of the natural population of Staphylococcus aureus: a comparison of multi-locus sequence typing with pulsed-field gel electrophoresis, randomly amplified polymorphic DNA analysis, and phage typing. J Clin Microbiol. 2002;40:4544-4546.

8. Hallin M, Denis O, Deplano A, De Mendonca R, De Ryck R, Rottiers S, Struelens MJ. Genetic relatedness between methicillin-susceptible and methicillin-resistant Staphylococcus aureus: results of a national survey. J Antimicrob Chemother. 2007;59:465-472.

9. Hanssen AM, Ericson Sollid JU. SCCmec in staphylococci: genes on the move. FEMS Immunol Med Microbiol. 2006;46:8-20.

10. Harmsen D, Claus H, Witte W, Rothganger J, Turnwald D, Vogel U. Typing of methicillin-resistant Staphylococcus aureus in a university hospital setting by using novel software for spa repeat determination and database management. J Clin Microbiol. 2003;41:5442-5448.

11. Hsu LY, Koh YL, Chlebicka NL, Tan TY, Krishnan P, Lin RT, Tee N, Barkham T, Koh TH. Establishment of ST30 as the predominant clonal type among community-associated methicillin-resistant Staphylococcus aureus isolates in Singapore. J Clin Microbiol. 2006;44:1090- 1093.

12. Huijsdens XW, van Santen-Verheuvel MG, Spalburg E, Heck ME, Pluister NG, Eijkelkamp BA, de Neeling AJ, Wannet WJ. Multiple cases of familial transmission of community-acquired methicillin-resistant Staphylococcus aureus. J Clin Microbiol. 2006;44:2994-2996.

13. Katayama Y, Robinson DA, Enright MC, Chambers HF. Genetic background affects stability of mecA in Staphylococcus aureus. J Clin Microbiol. 2005;43:2380-2383. 
14. Layer F, Ghebremedhin B, Konig W, Konig B. Heterogeneity of methicillin-susceptible Staphylococcus aureus strains at a German university hospital implicates the circulating-strain pool as a potential source of emerging methicillin-resistant S. aureus clones. J Clin Microbiol. 2006;44:2179-2185.

15. Lowy FD. Staphylococcus aureus infections. N Engl J Med. 1998;339: 520-532.

16. Melles DC, Gorkink RF, Boelens HA, Snijders SV, Peeters JK, Moorhouse MJ, van der Spek PJ, van Leeuwen WB, Simons G, Verbrugh HA, van Belkum A. Natural population dynamics and expansion of pathogenic clones of Staphylococcus aureus. J Clin Investig. 2004;114:1732-1740.

17. Mellmann A, Weniger T, Berssenbrugge C, Rothganger J, Sammeth M, Stoye J, Harmsen D. Based upon repeat pattern (BURP): an algorithm to characterise the long-term evolution of Staphylococcus aureus populations based on spa polymorphisms. BMC Microbiol. 2007;7:98.

18. Robinson DA, Enright MC. Evolution of Staphylococcus aureus by large chromosomal replacements. J Bacteriol. 2004;186:1060-1064.

19. Robinson DA, Enright MC. Evolutionary models of the emergence of methicillin-resistant Staphylococcus aureus. Antimicrob Agents Chemother. 2003;47:3926-3934.

20. Ruppitsch W, Indra A, Stoger A, Mayer B, Stadlbauer S, Wewalka G, Allerberger F. Classifying spa types in complexes improves interpretation of typing results for methicillin-resistant Staphylococcus aureus. J Clin Microbiol. 2006;44:2442-2448.

21. Strommenger B, Braulke C, Heuck D, Schmidt C, Pasemann B, Nubel U, Witte W. spa typing of Staphylococcus aureus as a frontline tool in epidemiological typing. J Clin Microbiol. 2008;46:574581.

22. Strommenger B, Kettlitz C, Weniger T, Harmsen D, Friedrich AW, Witte W. Assignment of Staphylococcus isolates to groups by spa typing, Smal macrorestriction analysis, and multi-locus sequence typing. J Clin Microbiol. 2006;44:2533-2540.

23. Tristan A, Bes $M$, Meugnier $H$, Lina G, Bozdogan B, Courvalin P, Reverdy ME, Enright MC, Vandenesch F, Etienne J. Global distribution of Panton-Valentine leukocidin-positive methicillinresistant Staphylococcus aureus, 2006. Emerg Infect Dis. 2007;13:594-600.

24. Vandenesch F, Naimi T, Enright MC, Lina G, Nimmo GR, Heffernan H, Liassine N, Bes M, Greenland $\mathrm{T}$, Reverdy ME, Etienne J. Community-acquired methicillin-resistant Staphylococcus aureus carrying Panton-Valentine leukocidin genes: worldwide emergence. Emerg Infect Dis. 2003;9:978-984.

25. Vivoni AM, Diep BA, de Gouveia Magalhaes AC, Santos KR, Riley LW, Sensabaugh GF, Moreira BM. Clonal composition of Staphylococcus aureus isolates at a Brazilian university hospital: identification of international circulating lineages. J Clin Microbiol. 2006;44:1686-1691. 



\section{Chapter 4: Genetic diversity of methicillin- resistant Staphylococcus aureus in a tertiary hospital in The Netherlands between 2002 and 2006}

E. Nulens, E.E. Stobberingh, E. Smeets, H. van Dessel, M.A. Welling, S. Sebastian, F.H. van Tiel, P.S. Beisser, R.H. Deurenberg

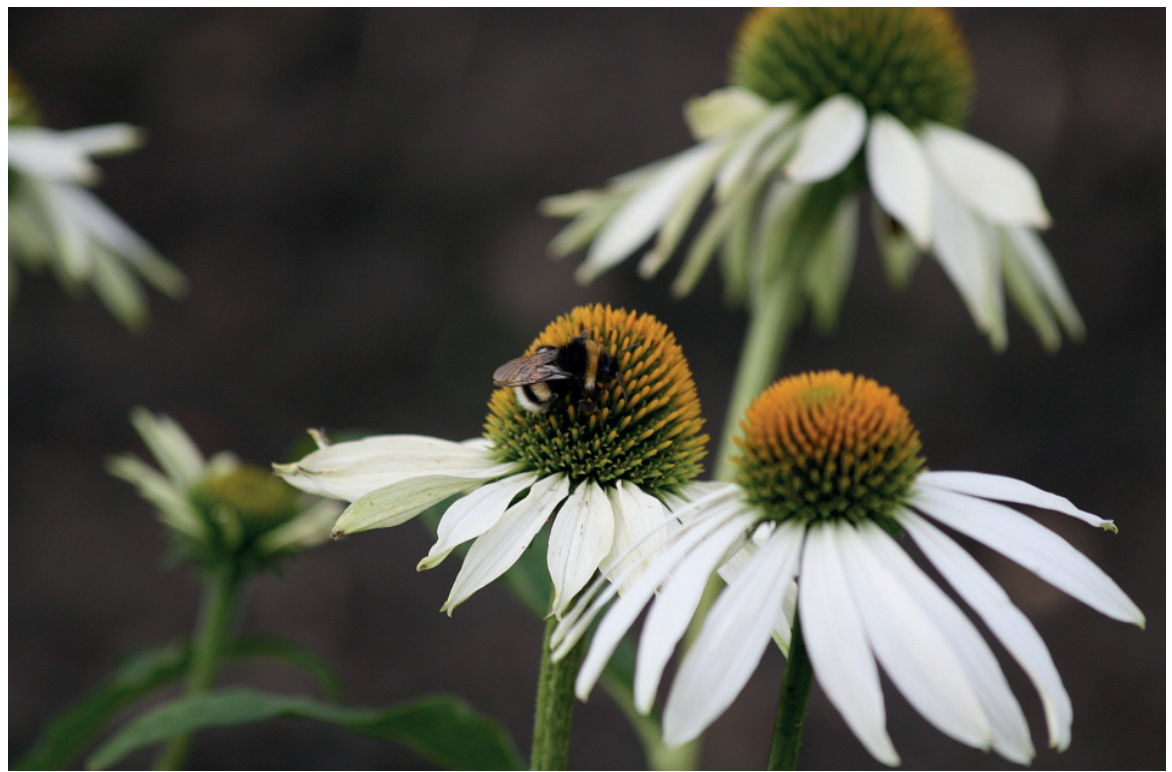

Echinacea purpurea White Swan, Brugge, 2009

\section{Published in}

European Journal of Clinical Microbiology \& Infectious Diseases. 2009;28:631-9.

(C) Springer-Verlag 2008 


\section{Abstract}

The aim of this study was to investigate the methicillin-resistant Staphylococcus aureus (MRSA) clones isolated in a Dutch university hospital, situated near the borders of Belgium and Germany, between 2002 and 2006. MRSA strains ( $n=175$ ) were characterised using spa and SCCmec typing. The presence of PantonValentine leukocidin (PVL) was determined. Between 2002 and 2005, ST5-MRSAIV was predominant, and the spa type of ST5-MRSA-IV changed from t002 to t447. ST5-MRSA-I, ST5-MRSA-II, ST228-MRSA-I, and ST247-MRSA-I were also observed in this period. From 2004, the MRSA genetic background became more diverse, and in 2006, ST5-MRSA-IV was only sporadically observed. From 2005, ST5-MRSA-II, ST8-MRSA-IV, ST22-MRSA-IV, and ST45-MRSA-IV were increasingly observed. Several other MRSA clones, such as ST239-MRSA-III, were found sporadically. Four PVL-positive MRSA isolates were observed, associated with ST80-MRSA-IV and ST8-MRSA-IV. ST5-MRSA-I, ST5-MRSA-II, ST5-MRSA-IV, and ST228-MRSA-I have not been described previously in The Netherlands. 


\section{Background}

Staphylococcus aureus can cause various infectious diseases, ranging from furuncles to postoperative wound infections [1]. Various clones of methicillinresistant S. aureus (MRSA) have disseminated, both in hospitals (hospitalassociated [HA] MRSA) and in the community (community-associated [CA] MRSA) [2]. However, the distinction between CA-MRSA and HA-MRSA is beginning to blur, since CA-MRSA clones have entered hospitals worldwide [3].

Resistance to methicillin and other ß-lactam antibiotics is coded by the mecA gene, which is situated on the mobile genetic element staphylococcal cassette chromosome mec (SCCmec) [4]. Seven main types of SCCmec (I to VII) and several variants have been distinguished today [5-7]. It has been suggested that SCCmec type IV or V, together with Panton-Valentine leukocidin (PVL) and a specific genetic background, are genetic markers for CA-MRSA [8]. The genetic background of $S$. aureus can be determined with spa typing. This involves sequencing of the short sequence repeat (SSR) region of the $S$. aureus protein $A$ (spa) gene [9]. The SSR region consists of several 24-bp repeats and its diversity is attributed to deletions, point mutations, and duplications of the repeats [10]. It has been shown that classifying spa types into spa-clonal complexes (spa-CCs) improves the interpretation of spa typing, and that spa typing, together with the algorithm based upon repeat pattern (BURP), is in accordance with typing results obtained by multi-locus sequence typing (MLST) and pulsed-field gel electrophoresis (PFGE) [11-14].

The prevalence of MRSA bloodstream isolates in The Netherlands is still low, but an increase has been observed in recent years, with a prevalence of $<1 \%$ at the beginning of the century to $2 \%$ in 2005 and 2006 [15, 16]. In addition, several reports have described CA-MRSA in The Netherlands $[17,18]$. Dutch hospitals at the border of Belgium and Germany experience the additional problem of crossborder health care-patients from Belgium and Germany cross the borders to visit health care facilities in The Netherlands. However, along with the patients, antibiotic-resistant micro-organisms, such as MRSA, can be imported into The Netherlands [19]. Several hospitals in the Euregion Meuse-Rhine (EMR), the border region between Belgium, Germany, and The Netherlands, have build up a strong collaboration during the last decade. For example, the Maastricht University Medical Center (MUMC) in Maastricht, The Netherlands and the German hospital in Aachen have an agreement for the transfer of patients for surgery, and consequently, dozens of patients are transferred each year between the two hospitals. In addition, around one hundred patients are transferred each year between the MUMC and the Belgian hospital in Tongeren. Any strategy to 
contain the transmission of MRSA requires knowledge of how MRSA is spread and the nature and the number of MRSA clones. In this study, spa and SCCmec typing were used to investigate the genetic background of MRSA strains isolated between 2002 and 2006 in a Dutch university hospital in order to detect shifts over time in the prevalence of major MRSA clones and the emergence of new MRSA clones.

\section{Methods}

\section{Bacterial isolates}

MRSA isolates ( $n=175)$ cultured between January 2002 and December 2006 in the MUMC, a tertiary care 715-bed university hospital situated near the borders of Belgium and Germany, were analysed. These MRSA isolates were comprised of 32 isolates from 2002, 29 isolates from 2003, 35 isolates from 2004, 39 isolates from 2005 and 40 isolates from 2006. One MRSA isolate per patient was included in the study. The majority $(n=174)$ of the MRSA isolates were derived from surveillance cultures from patients who were admitted to the hospital during the study period and were at risk for MRSA carriage according to the guidelines of the Dutch Workingparty on Infection Prevention (WIP) [20]. The source of the isolates was 59 (34\%) from the nose, 44 (25\%) from the throat, 36 (21\%) from the skin, 17 (10\%) from the perineum, 2 (1\%) from urine, and 17 (10\%) were of unknown origin. All isolates were identified as $S$. aureus by Gram stain, catalase, and coagulase testing [21].

MRSA strains COL, BK2464, ANS46, MW2, WIS, and HDE288 were used as reference strains for SCCmec type I, II, III, IV, V, and VI, respectively [22, 23]. MRSA cluster 28 was used as a reference strain for PVL [24].

\section{Antimicrobial susceptibility testing}

The susceptibility pattern of the MRSA isolates was determined according to the guidelines of the Clinical Laboratory Standards Institute (CLSI) using the microbroth dilution method with Mueller-Hinton II cation-adjusted broth (Becton Dickinson, USA), an inoculum of $5 \times 10^{5} \mathrm{CFU} / \mathrm{ml}$ and overnight incubation at $37^{\circ} \mathrm{C}$ [25]. The plates for the determination of the minimal inhibitory concentration (MIC) contained freeze-dried antibiotics (MCS Diagnostics, The Netherlands). Susceptibility to the following antimicrobial agents (range in $\mathrm{mg} / \mathrm{l}$ ) was determined: cefaclor (0.06-128), cefuroxime (0.06-128), clindamycin (0.03-64), ciprofloxacin (0.25-4), clarithromycin (0.03-64), gentamicin (0.06-64), linezolid (0.03-64), moxifloxacin (0.12-4), oxacillin (0.03-64), penicillin (0.004-8), rifampin 
(0.008-16), teicoplanin (0.06-128), tetracycline (0.03-64), trimethoprim/ sulfamethoxazole (0.015/0.29-32/680), and vancomycin (0.06-128). The susceptibility to fucidic acid and mupirocin (Rosco, Denmark) was determined using the disk-diffusion method [26, 27]. MRSA isolates resistant to clarithromycin were tested for inducible clindamycin resistance using the D-test [28].

\section{SCCmec typing}

SCCmec typing was essentially carried out as described by Oliveira et al. [22] with the following modification. The amplification of the mecA gene was performed using primers MecA147-F and MecA147-R (Sigma Genosys, UK), resulting in a PCR product of $147 \mathrm{bp}$ instead of $162 \mathrm{bp}$ [29]. It has been shown previously [30] that SCCmec type I elements which lack locus A (p/s region) are indistinguishable from SCCmec type IV elements with the method of Oliveira et al. [22]. Therefore, the $\mathrm{SCC}$ ec elements that were typed as SCCmec type IV with the method of Oliveira et al. [22], i.e. positive for mecA and locus $D$ (dcs region), were further analysed with the method of Ito et al. [23], i.e. the presence of ccrAB and Tn554 was investigated as described previously [19], and IS1272 was detected as described by Chongtrakool et al. [5]. The PCR reactions and the detection of the PCR products were performed as described previously [19].

\section{spa typing}

Real-time amplification of the spa locus, followed by sequencing of the SSR region was performed as described previously [31]. The spa types were clustered into spa-CCs with the algorithm BURP using the Ridom StaphType version 1.5 software package (http://www.ridom.de) with the settings recommended by the manufacturer. The associated clonal complexes (CCs), as determined with MLST, were allocated through the Ridom SpaServer (http:// spaserver.ridom.de).

\section{MLST}

To confirm the association between MLST and spa typing, in combination with BURP, MLST was performed on a representative set of strains of each major spa type and spa-CC (Table 1) as described previously [19]. 


\section{Calculation of diversity index and confidence intervals}

To assess the discriminatory power, Simpson's index of diversity (DI) and the 95\% confidence intervals $(\mathrm{Cl})$ for $\mathrm{SCCmec}$ and spa typing were calculated as described previously $[32,33]$.

\section{Detection of PVL}

The presence of PVL was investigated using a real-time PCR assay as described previously [24].

\section{Statistical analyses}

Statistically significant differences were calculated with the Mann-Whitney $U$ test using SPSS 15.0 (SPSS Inc., The Netherlands). A p value of $<0.05$ was considered statistically significant.

\section{Results}

\section{Antimicrobial susceptibility patterns}

All MRSA isolates were resistant to the ß-lactam antibiotics tested, i.e. cefaclor, cefuroxime, oxacillin, and penicillin, and were susceptible to linezolid, teicoplanin, and vancomycin. From 2002 to 2003, the percentage of MRSA isolates resistant to gentamicin increased and then decreased during the latter half of the study period. During the study period, the percentage of MRSA isolates resistant to fucidic acid decreased, while the percentage of MRSA isolates resistant to clarithromycin and mupirocin increased, being only significant for clarithromycin ( $p \leq 0.05$ ). A total of 36 (21\%) MRSA isolates (six from 2003, 18 from 2004, six from 2005, and six from 2006) were resistant to clarithromycin and susceptible to clindamycin. The D-test showed that 29 of these 36 MRSA isolates (81\%) had the inducible clindamycin resistance phenotype, i.e. four isolates from 2003, 17 from 2004, five from 2005, and three from 2006.

\section{Distribution of SCCmec elements}

The most prevalent SCCmec type was SCCmec type IV ( $n=103 ; 59 \%)$, followed by SCCmec type I ( $n=37 ; 21 \%)$, SCCmec type II ( $n=21 ; 12 \%)$, and SCCmec type III ( $n=8$; $5 \%)$. Ten of the SCCmec type I elements lacked the pls locus, and four of the 
SCCmec type IV elements lacked the dcs locus as determined with the method of Oliveira et al. [22].

From six MRSA isolates, the SCCmec type could not be determined. Four of these non-typeable SCCmec elements harbored only locus D according to the method of Oliveira et al. [22]. Furthermore, the isolates harbored both ccrAB1 and ccrAB2, and they were positive for IS1272 and negative for Tn554. Based on these findings, the SCCmec elements have properties of types I and IV. A fifth nontypeable SCCmec element harbored locus D and E as determined with the method of Oliveira et al. [22], and was positive for ccrAB2, IS1272, and Tn554. The last non-typeable SCCmec element did not harbor any of the loci as determined with the method of Oliveira et al. [22]; however, this isolate harbored ccrAB2, but was negative for both IS1272 and Tn554.

TABLE 1. Composition of the spa-CCs

\begin{tabular}{lccll}
\hline spa-CC & $\begin{array}{c}\text { No. (\%) } \\
\text { of } \\
\text { isolates }\end{array}$ & $\begin{array}{c}\text { No. (\%) } \\
\text { of } \text { spa } \\
\text { types }\end{array}$ & \multicolumn{1}{c}{ spa types $^{\mathrm{a}}$} & CC \\
\hline spa-CC001 & $103(59)$ & $9(28)$ & $\begin{array}{l}\text { t001, t002, t003, t041, t045, t067, t109, } \\
\text { t242, t447 }\end{array}$ & 5 \\
spa-CC008 & $26(15)$ & $6(19)$ & t008, t052, t064, t068, t303, t622 & 8 \\
spa-CC012 & $15(9)$ & $4(13)$ & t012, t037, t253, t1820 & $30,239^{b}$ \\
No founder 4 & $3(2)$ & $2(6)$ & t044, t131 & 80 \\
No founder 5 & $9(5)$ & $2(6)$ & t038, t740 & 45 \\
Singletons & $14(8)$ & $6(19)$ & t223, t346, t445, t690, t954, t1310 & $22,45^{c}$ \\
Excluded & $4(2)$ & $3(9)$ & t026, t111, t779 & $5,45^{\mathrm{e}}$ \\
NT & $1(1)$ & & & \\
Total & $175(100)$ & $\mathbf{3 2 ( 1 0 0 )}$ & & \\
\hline
\end{tabular}

NT: non-typeable

CC: clonal complex

${ }^{a}$ On strains with spa types in Boldface, MLST analyses was performed.

${ }^{\mathrm{b}}$ Four isolates were associated with CC239 (t037).

' Seven isolates were associated with CC22 (t223) and two isolates with CC45 (t445).

d spa types smaller than five spa repeats.

${ }^{\mathrm{e}}$ Two isolates were associated with CC5 (t111) and one isolate with CC45 (t026).

\section{Distribution of spa-CCs and MLST analyses}

Thirty-two spa types were found among the 175 MRSA isolates and these were grouped into five spa-CCs and six singletons. The three spa types that were excluded from the BURP analysis had a spa locus that was less than five spa 
repeats in length. One isolate could not be spa typed (Table 1). Two main spaCCs, spa-CC001 (59\% of the isolates) and spa-CC008 (15\% of the isolates), were found, associated with $\mathrm{CC} 5$ and $\mathrm{CC} 8$, respectively. Furthermore, spa-CC012, associated with CC 30 , was represented by $9 \%$ of the MRSA isolates, while a spaCC with no founder [5], associated with CC45, was represented by $5 \%$ of the isolates. The three isolates from the other spa-CC with no founder [4] were associated with the CA-MSRA ST80 clone (Table 1). From the 14 isolates that were classified as singletons, two were associated with CC45 (t445) and seven with CC22 (t223). Of the excluded spa types, two were associated with CC5 (t111) and one with CC45 (t026). MLST analyses confirmed the association between spaCC and MLST CC (Table 1).

\section{Discriminatory power}

The discriminatory power of the combination of spa and $\mathrm{SCCmec}$ typing was higher compared to spa typing alone. The other methods had a far less discriminatory power (Table 2).

TABLE 2. Discriminatory power of the different typing methods

\begin{tabular}{lccc}
\hline Typing method & Number of different groups & Diversity index & $\mathbf{9 5 \% ~ C l}$ \\
\hline spa typing & 32 & 0.888 & $0.861-0.916$ \\
spa typing/BURP & $11^{\mathrm{a}}$ & 0.600 & $0.522-0.678$ \\
SCCmec typing & $4^{\mathrm{b}}$ & 0.643 & $0.566-0.720$ \\
spa typing/SCCmec typing & $36^{\mathrm{c}}$ & 0.931 & $0.906-0.957$ \\
spa typing/BURP/SCCmec typing & $17^{\mathrm{d}}$ & 0.746 & $0.690-0.803$ \\
\hline
\end{tabular}

$\mathrm{Cl}$ : confidence interval

${ }^{a}$ Including the different singletons and excluding the excluded spa types.

${ }^{\mathrm{b}}$ Excluding the non-typeable $\mathrm{SCC}$ ec elements.

${ }^{c}$ Including the different singletons and excluding the non-typeable SCCmec elements.

d Including the different singletons and excluding the excluded spa types and the non-typeable SCCmec elements.

\section{Distribution of MRSA clones}

The Paediatric clone (ST5-MRSA-IV) was the most prevalent MRSA clone found between 2002 and 2005 (Figure 1). However, a shift in isolation frequency from spa type t002 to t447 was observed within the isolates associated with this clone (Figure 2). This shift was associated with the appearance of clarithromycin resistance, since $88 \%$ of the isolates typed as t 447 were resistant, while only $21 \%$ 
of the isolates typed as t002 were resistant. Other major MRSA clones, such as the Iberian clone (ST247-MRSA-I), the New York/Japan clone (ST5-MRSA-II), and the UK EMRSA-3 clone (ST5-MRSA-I) were observed during this period. During 2003, the Paediatric clone and the Southern Germany clone (ST228-MRSA-I) were almost equally distributed among the MRSA clones (Figure 1).

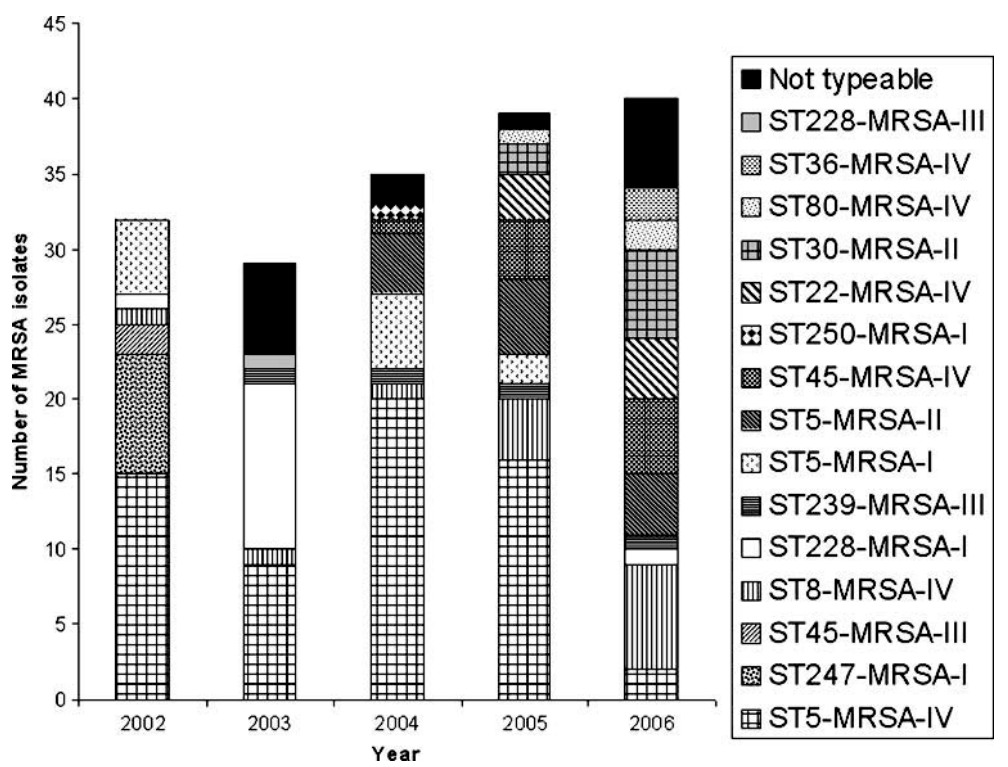

FIGURE 1. Distribution of methicillin-resistant Staphylococcus aureus (MRSA) clones in the MUMC between 2002 and 2006

From 2004, the genetic background of the MRSA clones became more diverse, and during 2006, the Paediatric clone was only sporadically observed (Figure 1). From 2005, the Berlin clone (ST45-MRSA-IV), the New York/Japan clone, the UK EMRSA-2/-6 clone (ST8-MRSA-IV), and the UK EMRSA-15 clone (ST22-MRSA-IV) were increasingly observed. The Brazilian/Hungarian clone (ST239-MRSA-III) was observed sporadically during the study period (Figure 1). One ST239-MRSA-III was isolated from an infection, and this strain caused a MRSA outbreak in 2003. Only the first isolate from this outbreak was included in the study.

Besides the major MRSA clones, several minor MRSA clones, such as the ST45MRSA-III clone, the ST228-MRSA-III clone, the ST30-MRSA-II clone and the ST36MRSA-IV clone, were observed during the study period (Figure 1). Fifteen of the 175 MRSA isolates (9\%) could not be related to a MRSA clone, either due to a 
non-typeable SCCmec element or to a spa type that was related to more than one MRSA clone.

\section{Prevalence of PVL}

Four of the 175 MRSA isolates (2\%) harbored PVL, as well as SCCmec type IV. These isolates, isolated in 2005 and 2006, had a genetic background associated with CA-MRSA. One isolate had spa type t044 and two isolates were spa typed as t131, and both spa types were associated with ST80 (European CA-MRSA clone). One isolate had spa type t622, associated with ST8 (USA300 clone). The patients from whom the strains were isolated suffered from skin problems, i.e. dermatitis, folliculitis, or cellulitis.

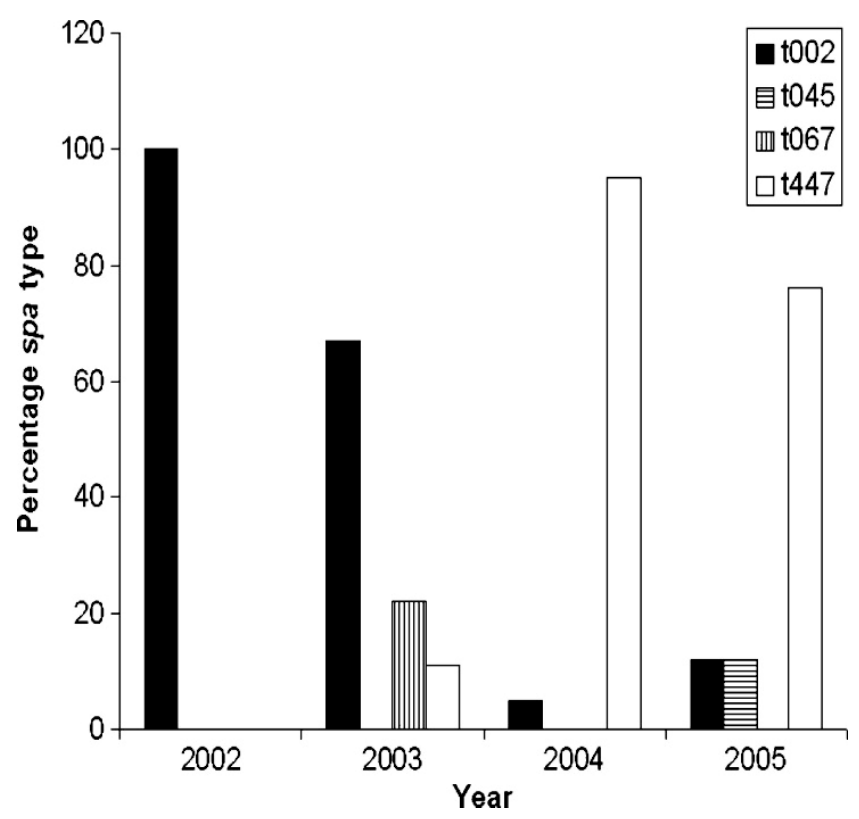

FIGURE 2. Shift from spa type t002 to t447 in the paediatric clone (ST5-MRSA-IV) between 2002 and 2005

\section{Discussion}

To control the transmission of MRSA in hospitals, it is important to study the nature and the number of MRSA clones present. This is the first study describing the nature of MRSA clones in a Dutch university hospital, situated near the borders of Belgium and Germany, over a five-year period. Between 2002 and 
2006, several major MRSA clones emerged and disappeared in our hospital, while other MRSA clones were found frequently. The composition of the MRSA population became more diverse during the latter part of the study period (Figure 1). Several MRSA clones were observed that have not been described previously in The Netherlands. In addition, a few PVL-positive MRSA isolates were observed.

It has been proposed that the combination of the genetic background and the SCCmec type should be used for the nomenclature of MRSA clones [34]. Typing of the spa locus has been shown to be a reliable method for long-term epidemiological surveillance studies, and spa typing/BURP data are in accordance with typing results obtained by MLST [12-14, 35]. The association between spaCC and MLST CC was confirmed in this study. However, spa typing should preferably be used in combination with additional genetic markers, such as SCCmec typing [35]. In our study, the highest discriminatory power for the combination of spa typing and SCCmec typing was observed.

Until 2006, the Paediatric clone (ST5-MRSA-IV) was the predominant MRSA clone in our hospital (Figure 1). Although this clone has previously been found in many European and South American countries, it has not been described before in The Netherlands [2]. The predominant spa types (t002 and t447) observed in the Paediatric clone in our hospital differ only in one spa repeat, i.e. r17 (http://spaserver.ridom.de). The deletion of spa repeat r17 of spa type t002 suggests that the Paediatric clone has adapted its fitness to a different niche. Another possibility is the introduction of a Paediatric clone with spa type 4447 into our hospital. Although several studies have described the replacement of a MRSA clone with another within a single hospital [36, 37], this is the first study that describes two major spa types within the same MRSA clone over time.

Other MRSA clones associated with CC5 observed in our hospital were the UK EMRSA-3 clone (ST5-MRSA-I), the New York/Japan clone (ST5-MRSA-II), and the Southern Germany (ST228-MRSA-I) clone. These clones have previously been observed in many countries worldwide, but not in The Netherlands [37]. Both ST5-MRSA-II and ST228-MRSA-I have been observed in Belgium and Germany [37]. Although ST228-MRSA-III isolates are rarely observed, several MRSA isolates with a non-typeable SCCmec type III element and a ST111 background, a single locus variant of ST228 at the pta locus, have been observed in Croatia [38]. Furthermore, ST85-MRSA-III isolates, like ST111 and ST228 associated with CC5, have been described previously [39]. This suggests that SCCmec type III can be harbored in CC5 MRSA isolates. 
A number of MRSA clones associated with CC8 were observed in this study. These included the Brazilian/Hungarian clone (ST239-MRSA-III), the Iberian clone (ST247-MRSA-I), and the UK EMRSA-2/-6 clone (ST8-MRSA-IV), which were all previously described in many European countries, including The Netherlands. The Archaic clone (ST250-MRSA-I) has not been described before in The Netherlands, but has been observed in some European countries, including Denmark and Germany [2].

During 2005, the UK EMRSA-15 clone (ST22-MRSA-IV) was observed for the first time in our hospital (Figure 1). This clone has previously been observed in many European countries and several Asian countries [2]. In The Netherlands, PVLpositive ST22-MRSA-IV isolates have been described previously [40]. Although it has been reported that the UK EMRSA-15 clone is commonly susceptible to tetracycline, in this study the majority of the isolates associated with this clone were tetracycline resistant [41].

Two minor MRSA clones associated with CC30 were observed that could fill a niche in the evolution of the UK EMRSA-16 clone (ST36-MRSA-II), e.g. eight ST30MRSA-II (spa type t012) and two ST36-MRSA-IV (spa type t253) isolates [2]. A ST30-MRSA-II isolate (spa type t012) has previously been found in Miami, Florida [42], while a PVL-positive CA-MRSA strain with a ST36-MRSA-IV background has been observed in Sweden [43]. Usually, the ST30 background is associated with SCCmec type IV and the ST36 background with SCCmec type II [44]. However, ST30-MRSA-II or ST36-MRSA-IV strains could be an intermediate step in the evolution of ST36-MRSA-II. There are two possibilities for the emergence of ST36MRSA-II. The first possibility is the acquisition of SCCmec type II in ST30-MSSA, followed by a point mutation in the pta locus, to become ST36-MRSA-Il; while, in the second possibility, ST30-MRSA-IV has evolved to ST36-MRSA-IV through a point mutation in the pta locus, and SCCmec type IV has subsequently been replaced by SCCmec type II [34, 44].

During 2000, the Berlin clone (ST45-MRSA-IV) emerged in The Netherlands [45], but only from 2004 onwards in our hospital (Figure 1). Two ST45-MRSA-III isolates were observed, but ST45 isolates usually harbor SCCmec type IV [34]. Recent studies have described a ST45-MRSA-I strain in The Netherlands [45] and a ST45MRSA-II strain in the United States [42]. Since the ST45 background has already been found in S. aureus strains from the 1970s [46], it is conceivable that several SCCmec elements, such as SCCmec type III and IV, have been introduced in S. aureus strains with a ST45 background over time, but that SCCmec type IV is the more stable SCCmec element in this genetic background [47]. 
Four major MRSA clones associated with MLST CC5 were observed during the study period that have not been described previously in The Netherlands, i.e. ST5-MRSA-I, ST5-MRSA-II, ST5-MRSA-IV, and ST228-MRSA-I. These MRSA clones could have been imported through cross-border health care into The Netherlands from health care facilities in Belgium and/or Germany [19]. The patient with the ST5-MRSA-I isolate was working in a long-term care facility in The Netherlands, a source observed previously for MRSA [48]. The ST5-MRSA-II isolate was observed in a patient suffering from cystic fibrosis (CF). A recent study showed that MRSA associated with CC5 have been observed in CF patients; however, that study showed that ST228-MRSA-I is predominant among CF patients [49]. The patients colonised with ST5-MRSA-IV and ST228-MRSA-I were both working in a Belgian hospital, suggesting import from a Belgian health care facility. The ST228-MRSA-I clone has been observed previously in Belgium [2]. Another possibility for the emergence of the MRSA clones could be the in vivo transfer of $\mathrm{SCCmec}$ from a methicillin-resistant coagulase negative staphylococcus to a methicillin-sensitive S. aureus (MSSA) [4]. Between 1999 and 2006, a total of 15 MSSA isolates associated with CC5 were observed in our hospital. Although it has been reported that the frequency of SCCmec transfer is rather low [31, 50], a recent study showed that the transfer of SCCmec into S. aureus associated with MLST CC5 is more frequent [51].

CA-MRSA usually harbors SCCmec type IV (or V) and often PVL, and it has been reported previously that the genetic background differs between continents, although this situation is beginning to blur [8]. Three CA-MRSA isolates were associated with the European CA-MRSA clone (ST80-MRSA-IV), while the fourth isolate was associated with the USA300 clone (ST8-MRSA-IV). Both CA-MRSA clones have previously been described in many European countries, including The Netherlands $[8,17,18]$.

No relation between the MRSA clone and the susceptibility pattern was observed, possible because resistance genes are not only carried on $\mathrm{SCCmec}$, but also on the genome, such as Tn554, as well as on plasmids [52]. Both transposons and plasmids are mobile and can thus be transferred to other $S$. aureus strains of different lineages, possibly due to antibiotic pressure.

The observation that some SCCmec type I elements lacked the pls locus, and are thus indistinguishable from SCCmec type IV elements with the method of Oliveira et al. [22], supports the implementation of the determination of ccrAB for the classification of SCCmec elements, as has been done recently [53]. Besides this updated method, two studies have described that sequencing of the $\operatorname{cr} B$ locus can be used for the determination of the SCCmec type [54, 55]. 
The SCCmec type of $3 \%$ of the MRSA isolates could not be determined with the method of Oliveira et al. [22]. Four non-typeable SCCmec elements harbored both cCrAB1 and ccrAB2. Earlier studies have described staphylococcal isolates that contained two ccr loci, i.e. ccrAB3 and $\operatorname{ccrC}$ in MRSA strain 85/2082 [5], ccrAB2 and ccrAB4 in S. epidermidis strain ATCC 12228 [56], and ccrAB2 and ccrC in MRSA strain $\mathrm{MRSA}_{\mathrm{ZH} 47}$ [57]. An encounter of two MRSA strains harboring different $\mathrm{SCC}$ ec elements could have led to the formation of a novel SCCmec element harboring two $\mathrm{ccr}$ loci, probably through homologues recombination. Two other MRSA isolates each harbored a different non-typeable SCCmec element. One isolate harbored ccrAB2, IS1272, and the dcs region, characteristic for SCCmec type IV. In addition, this SCCmec element harbored locus E [22] and Tn554, normally lacking in SCCmec type IV. This element could be a SCCmec type IV variant, harboring the region between integrated plasmid pl258 and Tn554. The last non-typeable SCCmec element contained only mecA and ccrAB2, normally present in SCCmec types II and IV. However, since this element neither possessed IS1272, mecl, nor Tn554, this element probably had a mec complex not usually associated with ccrAB2. Consequently, this SCCmec element could not be classified into one of the six SCCmec elements currently known [2, 54]. Further investigation into the structure of these $\mathrm{SCCmec}$ elements is required.

In summary, between 2002 and 2006, several major MRSA clones emerged and disappeared in our hospital, while other MRSA clones were found more or less repeatedly. In general, the composition of the MRSA population became more diverse during the latter years of the study. The Paediatric, the Southern Germany, the New York/Japan, and the UK EMRSA-3 clones have not been described previously in The Netherlands. Only a few CA-MRSA isolates were observed, associated with ST8-MRSA-IV and ST80-MRSA-IV. The great diversity of MRSA clones (which increased over time) in our hospital at the borders of Belgium and Germany stressed the importance of cross-border spread of antibiotic-resistant micro-organisms from Belgium and Germany into The Netherlands in our border region. Therefore, harmonisation of infection prevention protocols of the hospitals on both sides of the border is essential to control this problem. However, clonal diversity could also be explained by antibiotic consumption, or some other variable not searched for during this study. 


\section{Acknowledgements}

We thank H. de Lencastre and D.C. Oliveira from The Rockefeller University, New York, New York for providing the reference strains for SCCmec typing, as well as T. Ito from the Juntendo University, Tokyo, Japan, for providing the reference strain (WIS) for SCCmec V, and W. Wannet from the National Institute of Public Health and Environment (RIVM) in Bilthoven, The Netherlands, for the MRSA cluster 28 strain. Furthermore, we are grateful to R. Mohammed for MIC determinations.

\section{References}

1. Lowy FD. Staphylococcus aureus infections. $N$ Engl J Med. 1998;339:520-532. doi:10.1056/NEJM199808203390806

2. Deurenberg RH, Vink C, Kalenic S, Friedrich AW, Bruggeman CA, Stobberingh EE. The molecular evolution of methicillin-resistant Staphylococcus aureus. Clin Microbiol Infect. 2007;13:222-235. doi:10.1111/j.1469-0691.2006.01573.x

3. Otter JA, French GL. Nosocomial transmission of community-associated methicillin-resistant Staphylococcus aureus: an emerging threat. Lancet Infect Dis. 2006;6:753-755. doi:10.1016/S1473-3099(06)70636-3

4. Hanssen AM, Ericson Sollid JU. SCCmec in staphylococci: genes on the move. FEMS Immunol Med Microbiol. 2006;46:8-20. doi:10.1111/j.1574-695X.2005.00009.x

5. Chongtrakool P, Ito T, Ma XX, Kondo Y, Trakulsomboon S, Tiensasitorn C, Jamklang M, Chavalit T, Song JH, Hiramatsu K. Staphylococcal cassette chromosome mec ( $\mathrm{SCCmec}$ ) typing of methicillinresistant Staphylococcus aureus strains isolated in 11 Asian countries: a proposal for a new nomenclature for SCCmec elements. Antimicrob Agents Chemother. 2006;50:1001-1012. doi:10.1128/AAC.50.3.1001-1012.2006

6. Oliveira DC, Milheirico C, de Lencastre H. Redefining a structural variant of staphylococcal cassette chromosome mec, SCCmec type VI. Antimicrob Agents Chemother. 2006;50:3457-3459. doi:10.1128/AAC.00629-06

7. Takano T, Higuchi W, Otsuka T, Baranovich T, Enany S, Saito K, Isobe H, Dohmae S, Ozaki K, Takano M, Iwao Y, Shibuya M, Okubo T, Yabe S, Shi D, Reva I, Teng L, Yamamoto T. Novel characteristics of community-acquired methicillin-resistant Staphylococcus aureus belonging to multi-locus sequence type 59 in Taiwan. Antimicrob Agents Chemother. 2008;52:837-845. doi:10.1128/AAC.01001-07

8. Tristan A, Bes M, Meugnier H, Lina G, Bozdogan B, Courvalin P, Reverdy ME, Enright MC, Vandenesch F, Etienne J. Global distribution of Panton-Valentine leukocidin-positive methicillinresistant Staphylococcus aureus, 2006. Emerg Infect Dis. 2007;13:594-600.

9. Frenay HM, Bunschoten AE, Schouls LM, van Leeuwen WJ, Vandenbroucke-Grauls CM, Verhoef J, Mooi FR. Molecular typing of methicillin-resistant Staphylococcus aureus on the basis of protein A gene polymorphism. Eur J Clin Microbiol Infect Dis. 1996;15:60-64. doi:10.1007/BF01586186 
10. Shopsin B, Gomez M, Montgomery SO, Smith DH, Waddington M, Dodge DE, Bost DA, Riehman $\mathrm{M}$, Naidich S, Kreiswirth BN. Evaluation of protein A gene polymorphic region DNA sequencing for typing of Staphylococcus aureus strains. J Clin Microbiol. 1999;37:3556-3563.

11. Ruppitsch W, Indra A, Stoger A, Mayer B, Stadlbauer S, Wewalka G, Allerberger F. Classifying spa types in complexes improves interpretation of typing results for methicillin-resistant Staphylococcus aureus. J Clin Microbiol. 2006;44:2442-2448. doi:10.1128/JCM.00113-06

12. Strommenger B, Kettlitz C, Weniger T, Harmsen D, Friedrich AW, Witte W. Assignment of Staphylococcus isolates to groups by spa typing, Smal macrorestriction analysis, and multi-locus sequence typing. J Clin Microbiol. 2006;44:2533-2540. doi:10.1128/ JCM.00420-06

13. Strommenger B, Braulke C, Heuck D, Schmidt C, Pasemann B, Nubel U, Witte W. spa typing of Staphylococcus aureus as a frontline tool in epidemiological typing. J Clin Microbiol. 2008;46:574581. doi:10.1128/JCM.01599-07

14. Mellmann A, Weniger T, Berssenbrugge C, Keckevoet U, Friedrich AW, Harmsen D, Grundmann H. Characterisation of the clonal relatedness among the natural population of Staphylococcus aureus using spa sequence typing and the BURP algorithm. J Clin Microbiol. 2008;46:2805-2808. doi:10.1128/ JCM.00071-08

15. Tiemersma EW, Bronzwaer SL, Lyytikäinen O, Degener JE, Schrijnemakers P, Bruinsma N, Monen J, Witte W, Grundman H. Methicillin-resistant Staphylococcus aureus in Europe, 1999-2002. Emerg Infect Dis. 2004;10:1627-1634.

16. Wannet WJB, Huijsdens XW, Heck MEOC, Pluister GN, van Santen-Verheuvel MG, Spalburg S, van Luit M, Bosch T, Haenen A, Tiemersma EW, de Neeling AJ. Results of the Dutch MRSA surveillance program in 2005-2006. Infect Bul. 2007;18:347-351.

17. Wannet WJ, Spalburg E, Heck ME, Pluister GN, Tiemersma E, Willems RJ, Huijsdens XW, de Neeling AJ, Etienne J. Emergence of virulent methicillin-resistant Staphylococcus aureus strains carrying Panton-Valentine leukocidin genes in The Netherlands. J Clin Microbiol. 2005;43:33413345. doi:10.1128/ JCM.43.7.3341-3345.2005

18. Huijsdens XW, van Santen-Verheuvel MG, Spalburg E, Heck ME, Pluister GN, Eijkelkamp BA, de Neeling AJ, Wannet WJ. Multiple cases of familial transmission of community-acquired methicillin-resistant Staphylococcus aureus. J Clin Microbiol. 2006;44:2994-2996. doi:10.1128/JCM.00846-06

19. Deurenberg RH, Vink C, Oudhuis GJ, Mooij JE, Driessen C, Coppens G, Craeghs J, De Brauwer E, Lemmen S, Wagenvoort H, Friedrich AW, Scheres J, Stobberingh EE. Different clonal complexes of methicillin-resistant Staphylococcus aureus are disseminated in the Euregio Meuse-Rhine region. Antimicrob Agents Chemother. 2005;49:4263-4271. doi:10.1128/AAC.49.10.4263-4271.2005

20. MRSA Hospital. Dutch Workingparty on Infection Prevention. http://www.wip.nl. Accessed 17 December 2008.

21. Isenberg HD (ed) (2004) Clinical microbiology procedures handbook, 2nd edn. ASM Press, Washington.

22. Oliveira DC, de Lencastre H. Multiplex PCR strategy for rapid identification of structural types and variants of the mec element in methicillin-resistant Staphylococcus aureus. Antimicrob Agents Chemother. 2002;46:2155-2161. doi:10.1128/AAC.46.7.2155-2161.2002

23. Ito T, Ma XX, Takeuchi F, Okuma K, Yuzawa H, Hiramatsu K. Novel type V staphylococcal cassette chromosome mec driven by a novel cassette chromosome recombinase, ccrC. Antimicrob Agents Chemother. 2004;48:2637-2651. doi:10.1128/ AAC.48.7.2637-2651.2004 
24. Deurenberg RH, Vink C, Driessen C, Bes M, London N, Etienne J, Stobberingh EE. Rapid detection of Panton-Valentine leukocidin from clinical isolates of Staphylococcus aureus strains by real-time PCR. FEMS Microbiol Lett. 2004;240:225-228. doi:10.1016/j.femsle.2004.09.031

25. Clinical Laboratory Standards Institute (2007) Performance standards for antimicrobial susceptibility testing, methods for dilution antimicrobial susceptibility tests for bacteria that grow aerobically; approved standard-seventh edition: M7-A7. CLSI, Wayne, Pennsylvania, USA.

26. Toma E, Barriault D. Antimicrobial activity of fusidic acid and disk diffusion susceptibility testing criteria for Gram-positive cocci. J Clin Microbiol. 1995;33:1712-1715.

27. Fuchs PC, Jones RN, Barry AL. Interpretive criteria for disk diffusion susceptibility testing of mupirocin, a topical antibiotic. J Clin Microbiol. 1990;28:608-609.

28. O'Sullivan MVN, Cai Y, Kong F, Zeng X, Gilbert GL. Influence of disk separation distance on accuracy of the disk approximation test for detection of inducible clindamycin resistance in Staphylococcus spp. J Clin Microbiol. 2006;44:4072-4076. doi:10.1128/JCM.01632-06

29. Zhang K, McClure JA, Elsayed S, Louie T, Conly JM. Novel multiplex PCR assay for characterisation and concomitant subtyping of staphylococcal cassette chromosome mec types I to $\mathrm{V}$ in methicillin-resistant Staphylococcus aureus. J Clin Microbiol. 2005;43:5026-5033. doi:10.1128/JCM.43.10.5026-5033.2005

30. Shore A, Rossney AS, Keane CT, Enright MC, Coleman DC. Seven novel variants of the staphylococcal chromosomal cassette mec in methicillin-resistant Staphylococcus aureus isolates from Ireland. Antimicrob Agents Chemother. 2005;49:2070-2083. doi:10.1128/AAC.49.5.20702083.2005

31. Nulens E, Stobberingh EE, van Dessel H, Sebastian S, van Tiel FH, Beisser PS, Deurenberg RH. Molecular characterisation of Staphylococcus aureus bloodstream isolates collected in a Dutch university hospital between 1999 and 2006. J Clin Microboil. 2008;46:2438-2441. doi:10.1128/ JCM.00808-08

32. Hunter PR, Gaston MA. Numerical index of the discriminatory ability of typing systems: an application of Simpson's index of diversity. J Clin Microbiol. 1988;26:2465-2466.

33. Grundmann H, Hori S, Tanner G. Determining confidence intervals when measuring genetic diversity and the discriminatory abilities of typing methods for micro-organisms. J Clin Microbiol. 2001;39:4190-4192. doi:10.1128/JCM.39.11.4190-4192.2001

34. Enright MC, Robinson DA, Randle G, Feil EJ, Grundmann H, Spratt BG. The evolutionary history of methicillin-resistant Staphylococcus aureus (MRSA). Proc Natl Acad Sci USA. 2002:99:7687-7692. doi:10.1073/pnas.122108599

35. Hallin M, Deplano A, Denis O, De Mendonca R, De Ryck R, Struelens MJ. Validation of pulsed-field gel electrophoresis and spa typing for long-term, nationwide epidemiological surveillance studies of Staphylococcus aureus infections. J Clin Microbiol. 2007;45:127-133. doi:10.1128/JCM.0186606

36. Perez-Roth E, Lorenzo-Diaz F, Batista N, Moreno A, Mendez-Alvarez S. Tracking methicillinresistant Staphylococcus aureus clones during a 5-year period (1998 to 2002) in a Spanish hospital. J Clin Microbiol. 2004;42:4649-4656. doi:10.1128/JCM. 42.10.4649-4656.2004 
37. Velazquez-Meza ME, Aires De Sousa M, Echaniz-Aviles G, Solorzano-Santos F, Miranda-Novales G, Silva-Sanchez J, De Lencastre H. Surveillance of methicillin-resistant Staphylococcus aureus in a paediatric hospital in Mexico City during a 7-year period (1997 to 2003): clonal evolution and impact of infection control. J Clin Microbiol. 2004;42:3877-3880. doi:10.1128/ JCM.42.8.38773880.2004

38. Budimir A, Deurenberg RH, Plecko V, Vink C, Kalenic S, Stobberingh EE. Molecular characterisation of methicillin-resistant Staphylococcus aureus bloodstream isolates from Croatia. J Antimicrob Chemother. 2006;57:331--334. doi:10.1093/jac/ dki452

39. Aires de Sousa M, de Lencastre H. Evolution of sporadic isolates of methicillin-resistant Staphylococcus aureus (MRSA) in hospitals and their similarities to isolates of communityacquired MRSA. J Clin Microbiol. 2003;41:3806-3815. doi:10.1128/JCM.41. 8.3806-3815.2003

40. Wannet WJ, Heck ME, Pluister GN, Spalburg E, van Santen MG, Huijsdans XW, Tiemersma E, de Neeling AJ. Panton-Valentine leukocidin-positive MRSA in 2003: the Dutch situation. Euro Surveill. 2004;9:28-29.

41. Amorim ML, Faria NA, Oliveira DC, Vasconcelos C, Cabeda JC, Mendes AC, Calado E, Castro AP, Ramos $\mathrm{MH}$, Amorim JM, de Lencastre $\mathrm{H}$. Changes in the clonal nature and antibiotic resistance profiles of methicillin-resistant Staphylococcus aureus isolates associated with spread of the EMRSA-15 clone in a tertiary care Portuguese hospital. J Clin Microbiol. 2007;45:2881-2888. doi:10.1128/JCM.00603-07

42. Chung M, Dickinson G, De Lencastre H, Tomasz A. International clones of methicillin-resistant Staphylococcus aureus in two hospitals in Miami, Florida. J Clin Microbiol. 2004;42:542-547. doi:10.1128/JCM.42.2.542-547.2004

43. Soderquist B, Berglund C, Stralin K. Community-acquired pneumonia and bacteraemia caused by an unusual methicillin-resistant Staphylococcus aureus (MRSA) strain with sequence type 36, staphylococcal cassette chromosome mec type IV and Panton-Valentine leukocidin genes. Eur J Clin Microbiol Infect Dis. 2006;25:604-606. doi:10.1007/s10096-006-0195-y

44. Robinson DA, Kearns AM, Holmes A, Morrison D, Grundmann H, Edwards G, O'Brien FG, Tenover FC, McDougal LK, Monk AB, Enright MC. Re-emergence of early pandemic Staphylococcus aureus as a community-acquired methicillin-resistant clone. Lancet 2005;365:1256-1258. doi:10.1016/S0140-6736 (05)74814-5

45. Wannet WJ, Spalburg E, Heck ME, Pluister GN, Willems RJ, De Neeling AJ. Widespread dissemination in The Netherlands of the epidemic Berlin methicillin-resistant Staphylococcus aureus clone with low-level resistance to oxacillin. J Clin Microbiol. 2004;42:3077-3082. doi:10.1128/JCM.42.7.3077-3082.2004

46. Gomes AR, Westh $\mathrm{H}$, de Lencastre H. Origins and evolution of methicillin-resistant Staphylococcus aureus clonal lineages. Antimicrob Agents Chemother. 2006;50:3237-3244. doi:10.1128/AAC.00521-06

47. Katayama Y, Robinson DA, Enright MC, Chambers HF. Genetic background affects stability of mecA in Staphylococcus aureus. J Clin Microbiol. 2005;43:2380-2383. doi:10.1128/JCM.43. $5.2380-2383.2005$

48. Grossman S, Mager DD. Managing the threat of methicillin-resistant Staphylococcus aureus in home care. Home Healthc Nurse. 2008;26:356-364 quiz 365-356. doi:10.1097/ 01.NHH.0000324309.38286 .93 
49. Molina A, Del Campo R, Maiz L, Morosini MI, Lamas A, Baquero F, Canton R. High prevalence in cystic fibrosis patients of multi-resistant hospital-acquired methicillin-resistant Staphylococcus aureus ST228-SCCmecl capable of biofilm formation. J Antimicrob Chemother. 2008;62:961-967. doi:10.1093/ jac/dkn302

50. Robinson DA, Enright MC. Evolutionary models of the emergence of methicillin-resistant Staphylococcus aureus. Antimicrob Agents Chemother. 2003;47:3926-3934. doi:10.1128/AAC. 47.12.3926-3934.2003

51. Nubel U, Roumagnac $P$, Feldkamp $M$, Song JH, Ko KS, Huang YC, Coombs G, Ip M, Westh H, Skov R, Struelens MJ, Goering RV, Strommenger B, Weller A, Witte W, Achtman M. Frequent emergence and limited geographic dispersal of methicillin-resistant Staphylococcus aureus. Proc Natl Acad Sci USA. 2008;105:14130-14135. doi:10.1073/pnas.0804178105

52. Lindsay JA, Holden MT. Understanding the rise of the superbug: investigation of the evolution and genomic variation of Staphylococcus aureus. Funct Integr Genomics. 2006;6:186-201. doi:10.1007/s10142-005-0019-7

53. Milheirico C, Oliveira DC, de Lencastre H. Update to the multiplex PCR strategy for assignment of mec element types in Staphylococcus aureus. Antimicrob Agents Chemother. 2007;51:3374-3377. doi:10.1128/AAC.00275-07

54. Oliveira DC, Milheirico C, Vinga S, de Lencastre H. Assessment of allelic variation in the ccrAB locus in methicillin-resistant Staphylococcus aureus clones. J Antimicrob Chemother. 2006;58:2330. doi:10.1093/jac/dkl208

55. Lina G, Durand G, Berchich C, Short B, Meugnier H, Vandenesch F, Etienne J, Enright MC. Staphylococcal chromosome cassette evolution in Staphylococcus aureus inferred from ccr gene complex sequence typing analysis. Clin Microbiol Infect. 2006;12:1175-1184. doi:10.1111/j.14690691.2006.01548.x

56. Mongkolrattanothai K, Boyle S, Murphy TV, Daum RS. Novel non-mecA-containing staphylococcal chromosomal cassette composite island containing pbp4 and tagF genes in a commensal staphylococcal species: a possible reservoir for antibiotic resistance islands in Staphylococcus aureus. Antimicrob Agents Chemother. 2004;48:1823-1836. doi:10.1128/AAC.48.5.1823-1836. 2004

57. Heusser R, Ender M, Berger-Bachi B, McCallum N. Mosaic staphylococcal cassette chromosome mec containing two recombinase loci and a new mec complex, B2. Antimicrob Agents Chemother. 2007;51:390-393. doi:10.1128/AAC.00921-06 



\section{Chapter 5: Cross-border dissemination of methicillin-resistant Staphylococcus aureus, Euregio Meuse-Rhine region}

R. H. Deurenberg, E. Nulens, H. Valvatne, S. Sebastian, C. Driessen, J. Craeghs, E. De Brauwer, B. Heising, Y.J. Kraat, J. Riebe, F.S. Stals, T.A. Trienekens, J. Scheres, A.W. Friedrich, F.H. van Tiel, P.S. Beisser, E.E. Stobberingh

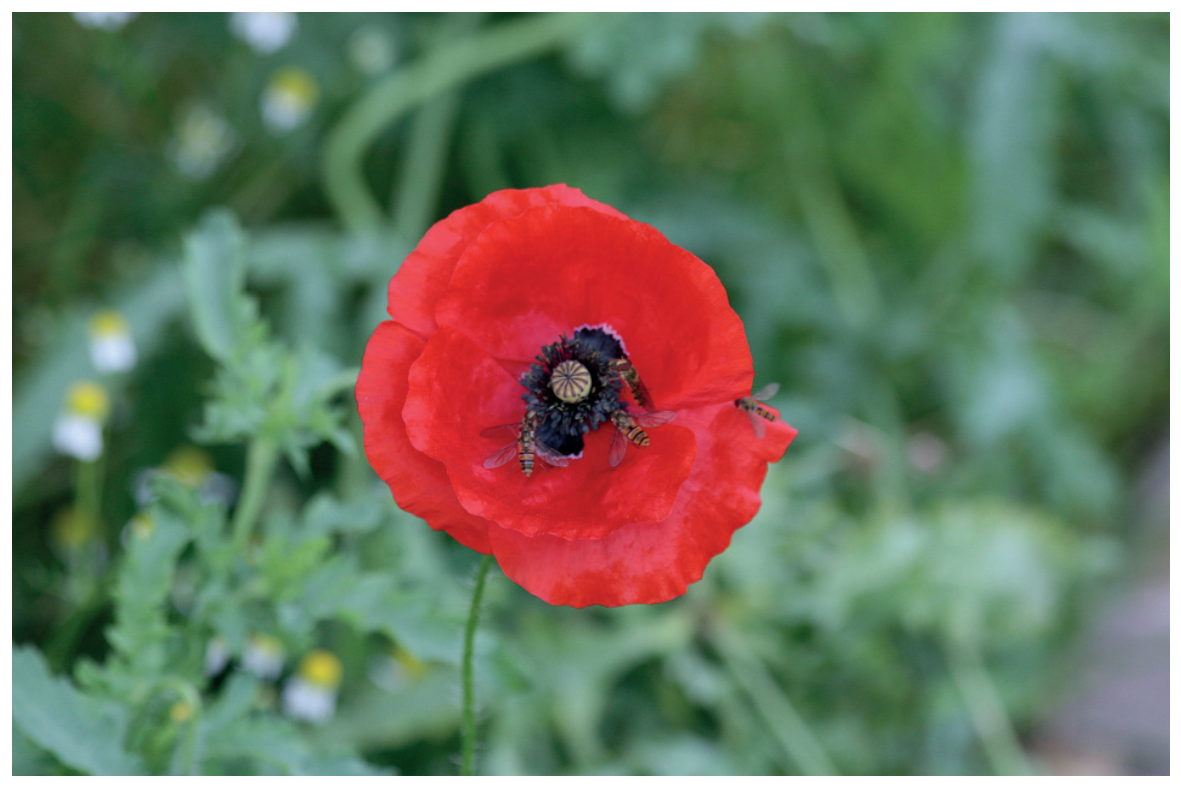

Papaver rhoeas, Brugge, 2009

\section{Published in}

Emerging Infectious Diseases. 2009;15:727-34.

The study was partly performed within the framework of the Interreg-III project "Cross-Border Health Care in the Euregio Meuse-Rhine." 


\section{Abstract}

Because the prevalence of methicillin-resistant Staphylococcus aureus (MRSA) differs among the 3 countries forming the Euregio Meuse-Rhine (EMR) region (Belgium, Germany, and the Netherlands), cross-border health care requires information about the spread of MRSA in the EMR. We investigated the emergence, dissemination, and diversity of MRSA clones in the EMR by using several typing methods. MRSA associated with clonal complexes 5, 8, 30, and 45 was disseminated throughout the EMR. Dutch isolates, mainly associated with sequence types (ST) ST5-MRSA-II, ST5-MRSA-IV, ST8-MRSA-IV, and ST45-MSRA-IV had a more diverse genetic background than the isolates from Belgium and Germany, associated with ST45-MRSA-IV and ST5-MRSA-II, respectively. MRSA associated with pigs (ST398-MRSA-IV/V) was found in the Dutch area of the EMR. Five percent of the MRSA isolates harbored Panton-Valentine leukocidin and were classified as community-associated MRSA associated with ST1, 8, 30, 80, and 89. 


\section{Introduction}

Almost one third of the European population lives in a border region (Euregio). These border regions have collaborated since the late 1950s, especially in the field of health care [1]. Cross-border patient mobility and free access to health care facilities within the European Union in general, and the Euregios in particular, are important for patients, medical doctors, health care facilities, and health care insurance companies. The Euregio Meuse-Rhine (EMR), an area totalling 4,973 square miles $\left(12,882 \mathrm{~km}^{2}\right)$, is the border region of Belgium, Germany, and the Netherlands. The EMR comprises the Belgian provinces of Limburg and Liège, the German-speaking region of Belgium, the Aachen region in Germany, and the southern part of the Dutch province of Limburg. Each year, thousands of the 3.88 million inhabitants of the EMR cross the border to consult a medical specialist or a health care facility. Since 2003, hospitals in the EMR have built a strong collaboration. For example, the University Hospital Maastricht in the Netherlands and the University Hospital Aachen in Germany have an official agreement for the transfer of patients; consequently, dozens of patients are transferred each year between the 2 hospitals. The same applies for the University Hospital Maastricht in the Netherlands and the General Hospital Vesalius in Belgium, between which nearly 100 patients are transferred each year. In an official publication of the European Commission (D. Byrne, Maastricht Conference on Cross-Border Health Care, Maastricht, the Netherlands, June 8, 2004), the EMR was mentioned as a model region for the European Union in the field of cross-border health care and cross-border cooperation of hospitals. Furthermore, in July 2008, establishment of a pan-European university hospital was announced, a collaboration among the university hospitals of Maastricht in the Netherlands and Aachen in Germany.

Of particular concern is cross-border dissemination of multidrug-resistant bacteria, such as methicillin-resistant Staphylococcus aureus (MRSA). The 3 countries forming the EMR differ considerably in the prevalence of hospitalisolated MRSA $(23.6 \%, 13.8 \%$, and $0.6 \%$ in Belgium, Germany, and the Netherlands, respectively) [2]. Consequently, cross-border transfer of patients may affect the dissemination and prevalence of MRSA, particularly when patients are transferred from countries with a relatively high prevalence to a country with a low prevalence.

A study of MRSA isolates from the EMR between December 1999 and February 2004 showed that isolates from clonal complex (CC) 5 and CC 8, which harbor the resistance elements staphylococcal cassette chromosome mec ( $\mathrm{SCCmec}$ ) types IIV, had been disseminated in the EMR [2]. Our aim was to investigate the 
emergence, dissemination, and diversity of MRSA clones in the EMR during a 10month period in 2005 and 2006 and to compare the results with those of the previous study. We used sequencing of the short sequence repeat (SSR) region of the $S$. aureus protein A gene (spa typing), multi-locus sequence typing (MLST), and SCCmec typing by PCR to investigate the genetic background of all MRSA isolates. The spa locus was typed to provide more detailed information about prevalent MRSA clones in the EMR, especially because the previous study used only MLST analyses on a small subset of isolates [2]. Finally, because an increase of Panton-Valentine leukocidin (PVL)-positive MRSA isolates in the Netherlands has recently been observed [3], we investigated the possible spread of PVLpositive MRSA clones into hospitals in the EMR, as well as the prevalence of the virulence factors collagen adhesion (CNA) and toxic shock syndrome toxin-1 (TSST-1).

\section{Materials and Methods}

\section{MRSA Isolates}

We investigated 257 MRSA isolates, cultured during July 2005-April 2006 from 8 geographically closely related hospitals in the EMR. The hospitals included 1 hospital in Belgium (General Hospital Vesalius, Tongeren, 355 beds), 2 hospitals in Germany (General Hospital Dūren, 521 beds, and Marien Hospital, Aachen, 321 beds), and 5 hospitals in the Netherlands (Atrium Medical Center, Heerlen, 811 beds; Orbis Medical and Care Center, Sittard, 578 beds; Laurentius Hospital, Roermond, a 458-bed general hospital; University Hospital Maastricht, a tertiary hospital, 680 beds; and VieCuri Medical Center, Venlo, a 554-bed general hospital). The 257 MRSA isolates comprised 44 from Belgium, 92 from Germany, and 121 from the Netherlands. Isolates from the Belgian and German hospitals were from patients with MRSA infection; Dutch isolates were from patients carrying MRSA who were admitted to the Dutch hospitals. All isolates were identified as $S$. aureus by Gram stain, catalase, and coagulase testing. The presence of the mecA gene was determined as described previously [2].

\section{Antimicrobial Drug Susceptibility Testing}

The susceptibility pattern of the MRSA isolates was determined according to the guidelines of the Clinical and Laboratory Standards Institute [4]. Susceptibility to the following antimicrobial agents was determined as MIC: cefaclor, cefuroxime, clindamycin, ciprofloxacin, clarithromycin, gentamicin, linezolid, moxifloxacin, oxacillin, penicillin, rifampin, teicoplanin, tetracycline, trimethoprim/ sulfamethoxazole, and vancomycin. The susceptibility to fucidic acid and 
mupirocin (Rosco, Taastrup, Denmark) was determined by using the disk-diffusion method $[5,6]$. MRSA isolates resistant to clarithromycin were tested for inducible clindamycin resistance by using the D-test [7].

\section{Typing Methods}

SCCmec typing was performed as described by Oliveira et al. [8] with the modification described previously [2]. SCCmec type I elements that lack locus A (p/s region) are indistinguishable [9] from SCCmec type IV elements when the method of Oliveira et al. is used [8]. Furthermore, locus D (dcs region) is detected in both SCCmec types IV and VI [10]. Therefore, SCCmec elements that were typed as SCCmec type IV using the method of Oliveira et al. [8] were further analysed for presence of the $c c r A B$ gene. SCCmec elements that could not be typed with the method of Oliveira et al. [8] were further analysed by using the methods of Ito et al. [11] and Zhang et al. [12].

Real-time amplification of the spa gene was performed as described previously, followed by sequencing of the SSR region [13]. The spa types were clustered into spa-CCs using the algorithm Based Upon Repeat Pattern (BURP) with the Ridom StaphType version 1.4 software package (www.ridom.de). Because spa typing, together with the algorithm BURP, yields results concordant with typing results obtained by MLST and pulsed-field gel electrophoresis [13], the associated CCs, as determined with MLST, were allocated through the Ridom SpaServer (http://spaserver.ridom.de). To confirm the association between MLST and spa typing, in combination with BURP, MLST was performed on a representative set of 12 strains of each major spa type and spa-CC [2]. The presence of CNA, PVL, and TSST-1 was determined with real-time PCR assays [14, 15].

\section{Results}

\section{Antimicrobial Drug Susceptibility Patterns}

All 257 MRSA isolates were resistant to the $\beta$-lactam antimicrobial agents cefaclor, cefuroxime, oxacillin, and penicillin and were susceptible to linezolid, teicoplanin, and vancomycin. Most isolates were also resistant to ciprofloxacin (84\%) and moxifloxacin (82\%). The Dutch MRSA isolates were more often susceptible to ciprofloxacin and moxifloxacin than were the Belgian and German isolates (Table 1) $(p<0.05)$. Furthermore, $78 \%$ of the MRSA isolates were resistant to clarithromycin, and $62 \%$, to clindamycin. Susceptibility for clarithromycin and clindamycin differed by country (Table 1). A total of 41 MRSA isolates (19 from 
Belgium, 5 from Germany, and 17 from the Netherlands) was resistant to clarithromycin and susceptible to clindamycin. The D-test showed that 31 (76\%) of these $41 \mathrm{MRSA}$ isolates had the inducible clindamycin resistant phenotype, including 15 from Belgium, 5 from Germany, and 11 from the Netherlands.

TABLE 1. Non- $\beta$-lactam antimicrobial drug resistance patterns of 257 methicillin-resistant Staphylococcus aureus (MRSA) isolates in the Euregio Meuse-Rhine region, July 2005-April 2006

\begin{tabular}{lccccccccccc}
\hline & No. & \multicolumn{10}{c}{ No. (\%) resistant MRSA isolates } \\
\cline { 2 - 11 } Country & Isolates & CIP & MXF & CLI & GEN & CLR & SXT & TET & RIF & MUP & FUC \\
\hline Belgium & 44 & $44(100)$ & $43(98)$ & $5(11)$ & $2(5)$ & $24(55)$ & 0 & $4(9)$ & 0 & $4(9)$ & 0 \\
Germany & 92 & $89(97)$ & $89(97)$ & $78(85)$ & $5(5)$ & $83(90)$ & 0 & $3(3)$ & $2(2)$ & $1(1)$ & $1(1)$ \\
The Netherlands & 121 & $84(69)$ & $79(65)$ & $76(63)$ & $11(9)$ & $93(77)$ & $3(2)$ & $22(18)$ & 0 & $8(7)$ & $1(1)$ \\
Total & $\mathbf{2 5 7}$ & $\mathbf{2 1 7 ( 8 4 )}$ & $\mathbf{2 1 1 ( 8 2 )}$ & $\mathbf{1 5 9 ( 6 2 )}$ & $\mathbf{1 8 ( 7 )}$ & $\mathbf{2 0 0 ( 7 8 )}$ & $\mathbf{3 ( 1 )}$ & $\mathbf{2 9 ( 1 2 )}$ & $\mathbf{2 ( 1 )}$ & $\mathbf{1 3 ( 5 )}$ & $\mathbf{2 ( 1 )}$ \\
\hline
\end{tabular}

MRSA: methicillin-resistant Staphylococcus aureus

CIP: ciprofloxacin

MXF: moxifloxacin

CLI: clindamycin

GEN: gentamicin

CLR: clarithromycin

SXT: trimethoprim/sulfamethoxazole

TET: tetracycline

RIF: rifampin

MUP: mupirocin

FUC: fucidic acid

No isolates showed resistance to linezolid, vancomycin, or teicoplanin.

\section{Distribution of MRSA Clones}

SCCmec type IV was predominant in MRSA isolates from Belgium (77\%), whereas MRSA isolates from Germany harbored mainly SCCmec type II (82\%). MRSA isolates from the Dutch region harbored both SCCmec type II and IV (27\% and $65 \%$, respectively). Although $25(10 \%)$ of the 257 MRSA isolates harbored a SCCmec element that could not be typed with the method of Oliveira et al. [8], they could be typed with the other methods. Seven MRSA isolates from Belgium harbored a SCCmec type III element that lacked Tn554, which is usually characteristic for SCCmec type III. From the German region, 1 isolate that had a non-typeable SCCmec element harbored ccrC, locus E, and Tn554. The method of Zhang et al. [12] classified this element as SCCmec type III. In the Netherlands, 17 MRSA isolates contained a non-typeable SCCmec element as defined by Oliveira et al. [8]. Ten of these were classified as SCCmec type IV, lacking locus D. The 
remaining 7 harbored $c c r C$, characteristic for SCCmec type $V$, and were classified as such with the method of Zhang et al. [12].

The 257 MRSA isolates were classified into 36 different spa types, and BURP analysis showed 6 spa-CCs, 4 singletons, and 3 spa types that were excluded from the analysis because the spa region was <5 spa repeats long (Table 2). MLST analyses showed 10 different STs among the 12 MRSA strains (Table 2). In the EMR, spa-CC 045 (MLST CC5; 21\%) and spa-CC 038 (MLST CC45; 75\%) were found predominantly among MRSA isolates from the Belgian region; spa-CC 045 (MLST CC5; 85\%) was found among isolates from the German region. The Dutch MRSA isolates were grouped into spa-CCO45 (MLST CC5; 39\%), spa-CC 019/012/318/011/108 (MLST CC30 and CC398; 15\%), spa-CC038 (MLST CC45; $15 \%)$, spa-CC with no founder 5 (MLST CC8; 16\%), and spa-CC with no founder 6 (MLST CC45; 5\%).

The ST5-MRSA-II (New York/Japan) clone was found mainly in Germany and the Netherlands, and the ST45-MRSA-IV (Berlin) clone was found in Belgium and the Netherlands. Furthermore, the ST5-MRSA-IV (Paediatric) clone was found among the Dutch isolates. The MRSA isolates classified as CC30 (ST30-MRSA-IV and ST36MRSA-II) were found only in the Netherlands. Most of the ST8-MRSA-IV (UK EMRSA-2/6) isolates were found in the Netherlands. Furthermore, several ST398MRSA-IV and ST398-MRSA-V isolates were found in certain Dutch hospitals (Figure 1; Table 3).

\section{Prevalence of Virulence Factors}

Eleven (5\%) of the 257 MRSA isolates were PVL-positive. These isolates were associated with different genetic backgrounds, i.e., ST1-MRSA-V (1 Dutch isolate), ST8-MRSA-IV, ST30-MRSA-IV (2 Dutch isolates each), ST45-MRSA-IV (1 isolate from Germany), ST80-MRSA-IV (1 isolate from Germany and 2 from the Netherlands), ST89-MRSA-IV and ST89-MRSA-V (1 Dutch isolate each). Six of the PVL-positive isolates were positive for the cna gene, and none harbored the tst gene.

Nine (4\%) of the 257 MRSA isolates were positive for the tst gene, 4 isolates were classified as ST22-MRSA-IV, 3 as ST36-MRSA-II, 1 as ST30-MRSA-IV, and 1 could not be classified as a MRSA clone (spa type t779). All isolates were from the Netherlands and were positive for the cna gene; none harbored PVL.

Ninety-five (37\%) of the 257 MRSA isolates were positive for the cna gene (34 from Belgium, 9 from Germany, and 52 from the Netherlands). All MRSA isolates 
from CC30, CC45, and ST398 harbored the cna gene. Furthermore, 1 isolate from CC5, 1 from CC80, 6 classified as singletons (associated with ST22 and ST89), and 2 excluded from the BURP analyses were positive for the cna gene.

TABLE 2. Composition of the spa-CCs of 257 methicillin-resistant Staphylococcus aureus (MRSA) isolates in the Euregio Meuse-Rhine region, July 2005-April 2006

\begin{tabular}{|c|c|c|c|c|c|}
\hline spa-CC & $\begin{array}{l}\text { No. (\%) } \\
\text { isolates }\end{array}$ & $\begin{array}{c}\text { No. (\%) } \\
\text { spa } \\
\text { types }\end{array}$ & spa types & ST & $\mathrm{CC}$ \\
\hline 045 & $134(52)$ & $9(25)$ & $\begin{array}{l}\text { t002, t003, t041, t045, t179, } \\
\text { t447, t504, t838, t1107 }\end{array}$ & ST5/ST225 ${ }^{\mathrm{a}}$ & CC5 \\
\hline 019/012/318/011/108 & $19(7)$ & $7(19)$ & $\begin{array}{l}\text { t011, t012, t019, t034, t108, } \\
\text { t318, t582 }\end{array}$ & ST36/ST398 & СС30/CC398 \\
\hline 038 & $58(22)$ & $5(14)$ & $\begin{array}{l}\text { t038, t161, t740, t1288, } \\
\text { t1310 }\end{array}$ & ST45 & CC45 \\
\hline $044 / 042$ & $4(2)$ & $4(11)$ & $\mathrm{t} 042, \mathrm{t} 044, \mathrm{t} 131, \mathrm{t} 345$ & ST728/ST772 ${ }^{\mathrm{C}}$ & $\mathrm{CC} 1 / \mathrm{CC} 80$ \\
\hline No founder 5 & $22(9)$ & $2(6)$ & t008, t622 & ST8 & CC8 \\
\hline No founder 6 & $8(3)$ & $2(6)$ & t040, t553 & ST45 & CC45 \\
\hline Singletons & $8(3)$ & $4(11)$ & t223, t375, t682, t786 & ST $217^{d}$ & CC22/CC89 \\
\hline Excluded $^{e}$ & $4(2)$ & $3(8)$ & $\mathrm{t} 457, \mathrm{t} 779, \mathrm{t} 1011$ & & \\
\hline Total & $257(100)$ & $36(100)$ & & & \\
\hline
\end{tabular}

CC: clonal complex

MRSA: methicillin-resistant Staphylococcus aureus

ST: sequence type.

Boldface indicates spa types on which multi-locus sequence typing analysis was performed.

${ }^{a}$ The strains spa typed as t003 and t045 had ST225, a single-locus variant of ST5 at the tpi locus. The strain spa typed as $\mathrm{t} 447$ had ST5.

${ }^{\mathrm{b}}$ The strain spa typed as t011 had ST398, and the strain spa typed as t012 had ST36.

' The strain spa typed as t044 had ST728, a single-locus variant of ST80 at the tpi locus. The strain spa typed as $\mathrm{t} 345$ had ST772, a single-locus variant of ST1 at the pta locus.

${ }^{\mathrm{d}}$ The strain spa typed as t223 had ST217, a single-locus variant of ST22 at the tpi locus.

e spa types with $<5$ spa repeat.

\section{Discussion}

Because cross-border health care is an issue in the EMR, and the prevalence of MRSA differs among the countries forming the EMR, studying the possible emergence, spread, and diversity of MRSA clones within and among these countries is important [2]. In addition to MRSA clones from CC5 and CC8, found previously in the EMR, we observed MRSA isolates from CC30 and CC45. Furthermore, the Dutch isolates had a more heterogeneous genetic background than did MRSA isolates from Belgium and Germany. The prevalence of PVL- 
positive MRSA isolates, belonging to ST1, 8, 30, 80 and 89 , was higher than that found in the previous study (5\% vs. $1.3 \%$ ) [2].

The antimicrobial susceptibility of the MRSA isolates depends on the $S$. aureus lineage. The observation that the Dutch MRSA isolates were more often susceptible to ciprofloxacin and moxifloxacin than were isolates from Belgium and Germany can be explained by the fact that the isolates associated with ST5MRSA-IV, ST22-MRSA-IV, and ST30-MRSA-IV, which were susceptible to ciprofloxacin and moxifloxacin, were mainly observed in the Netherlands. Although ST22-MRSA-IV is commonly susceptible to tetracycline, the ST22-MRSAIV isolates in this study were resistant to tetracycline [16]. S. aureus can harbor resistance genes on mobile genetic elements on the genome, such as $\operatorname{Tn} 554$, as well as on plasmids, and these can be exchanged among $S$. aureus lineages, possibly because of antimicrobial drug pressure [17].

Primarily because of the Dutch "search-and-destroy" policy, isolates derived from colonised persons were available from the Netherlands, whereas isolates from Belgium and Germany were derived from infections. However, nasal carriers are at increased risk of acquiring MRSA infection [18]. Consequently, not preventing the spread of MRSA among nasal carriers could lead to MRSA infection among these persons. Furthermore, the molecular epidemiology of MRSA can vary widely among hospitals. In the Dutch hospitals of the EMR, MRSA clones in each hospital were diverse, whereas in the Belgian hospital and 2 German hospitals, 1 MRSA clone predominated, showing that the number of hospitals is unlikely to have biased the results of our study.

Most of the MRSA isolates from Belgium were associated with the Berlin clone (ST45-MRSA-IV). This clone has previously been found in Belgium, Germany, and the Netherlands [19]. Most of the MRSA isolates from Germany were associated with the New York/Japan clone (ST5-MRSA-II), previously found in Belgium and Germany $[2,19]$. Most of the Dutch MRSA isolates belonged to 5 MRSA clones (Table 3). Twenty-five percent of the Dutch isolates were associated with the New York/Japan clone (ST5-MRSA-II), which has not been previously found in the Netherlands. The Paediatric clone (ST5-MRSA-IV), which represented $14 \%$ of the Dutch isolates, has been found in Belgium but not in the Netherlands [20, 21]. The Berlin clone (ST45-MRSA-IV), comprising $21 \%$ of the Dutch isolates, and the UK EMRSA-2/-6 clone (ST8-MRSA-IV), comprising 16\% of the Dutch isolates, have been described in all 3 EMR countries $[19,20]$. In addition, some less prevalent MRSA clones were observed. Four tst-positive MRSA isolates were associated with the UK EMRSA-15 clone (ST22-MRSA-IV), previously found in Belgium and Germany but not in the Netherlands $[19,20]$. Three Dutch MRSA isolates (spa 
type t012), harboring SCCmec type II, were associated with the CC30 lineage. These isolates might be derived from the UK EMRSA-16 (ST36-MRSA-II) clone (spa type t018) because spa types t012 and t018 differ in 1 spa repeat ( 24 ) and are thus related. Furthermore, both clones harbor the $c n a$ and tst genes [22, 23]. The highly endemic UK EMRSA-16 clone has not been observed before in the Netherlands, although this clone has previously been found in Belgium [24]. Seven and 5 of the Dutch MRSA isolates were associated with ST398-MRSA-IV and ST398-MRSA-V, respectively, MRSA clones usually observed in pigs and among screening samples from pig farmers [25]. The ST398 clone is now observed among screening samples of veterinarians from many countries in Europe, including Belgium and Germany [26]. However, ST398 also has been isolated from several forms of human infections in Germany [27]. The ST398 isolates from our study were positive for the cna gene, suggesting a higher virulence than that of the CNA-negative German ST398 MRSA isolates [27]. One Dutch MRSA strain was associated with the ST30-MRSA-IV clone, previously reported in Belgium, Germany, and France [20, 21, 28]. The more diverse genetic background among MRSA isolates in the Dutch part of the EMR and the close cooperation of hospitals in the EMR might suggest that importation of MRSA from Belgium and Germany has occurred through cross-border health care (Table 4) [2]. Other, less likely, explanations for the diversity of MRSA clones in the Netherlands are the spread of MRSA from countries other than Belgium or Germany [19] and the emergence of new MRSA clones in vivo through transfer of the SCCmec element from methicillin-resistant coagulase-negative staphylococci to methicillin-sensitive $S$. aureus strains [29].

We could not determine the SCCmec type for $10 \%$ of the MRSA isolates by using the method of Oliveira et al. [8]. This percentage was similar to that found in other studies [30,31] but higher than the 3\% previously found in the EMR [2]. The relatively large number of non-typeable SCCmec types found in this study, probably formed by homologous recombination among SCCmec elements, supports the need for a new system for SCCmec typing and nomenclature [19].

The 7 Belgian MRSA isolates with the non-typeable SCCmec type III element were associated with CC5 and had the related spa types t045 and t1107 (http://spaserver.ridom.de). Although SCCmec type III usually is found in the CC8 genetic background, such as in the ST239-MRSA-III clone, a MRSA associated with CC5 (spa type t045) and harboring SCCmec type III recently was observed in Belgium [32]. This might suggest that a new MRSA clone, ST5-MRSA-III, is beginning to emerge in Belgium. 
The non-typeable SCCmec element of the German MRSA isolate harbored locus E and ccrC, specific for SCCmec type V [2], and Tn554, normally carried by SCCmec type II, III, and SCCmercury. Zhang et al. [12] classified this element as SCCmec type III, but the SCCmec type III-specific primers used by this method are situated near locus E on SCCmercury [33], indicating that this element could be a SCCmercury element containing mecA. Further investigation is needed into the structure of this element.

TABLE 3. Distribution of methicillin-resistant Staphylococcus aureus (MRSA) clones in the Euregio Meuse-Rhine region, by country, July 2005-April 2006

\begin{tabular}{|c|c|c|c|c|}
\hline \multirow[b]{2}{*}{ MRSA clone } & \multicolumn{4}{|c|}{ No. Isolates } \\
\hline & Belgium & Germany & The Netherlands & Total \\
\hline ST1-MRSA-V & & & 1 & 1 \\
\hline ST5-MRSA-I & 1 & 1 & & 2 \\
\hline ST5-MRSA-II & 1 & 75 & 30 & 106 \\
\hline ST5-MRSA-IV & & 2 & 17 & 19 \\
\hline ST8-MRSA-IV & 1 & 2 & 19 & 22 \\
\hline ST22-MRSA-IV & & & 4 & 4 \\
\hline ST30-MRSA-IV & & & 3 & 3 \\
\hline ST36-MRSA-II & & & 3 & 3 \\
\hline ST45-MRSA-IV & 33 & 8 & 25 & 66 \\
\hline ST80-MRSA-IV & & 1 & 2 & 3 \\
\hline ST89-MRSA-I & & & 1 & 1 \\
\hline ST89-MRSA-V & & & 1 & 1 \\
\hline ST398-MRSA-IV & & & 7 & 7 \\
\hline ST398-MRSA-V & & & 5 & 5 \\
\hline NT MRSA & $7^{\mathrm{a}}$ & $1^{b}$ & $2^{c}$ & 10 \\
\hline Excluded $^{d}$ & 1 & 2 & 1 & 4 \\
\hline Total & 44 & 92 & 121 & 257 \\
\hline
\end{tabular}

MRSA: methicillin-resistant Staphylococcus aureus

ST: sequence type

NT: non-typeable

Based on spa and Staphylococcus cassette chromosome (SCC) mec typing.

${ }^{a}$ These strains were classified into clonal complex (CC) 5 and had a non-typeable SCCmec type III element.

${ }^{\mathrm{b}}$ This strain had a non-typeable SCCmec element belonging to CC30.

${ }^{\mathrm{C}}$ These strains harbored SCCmec type IV and could not be classified into a CC.

${ }^{\mathrm{d}}$ spa types with $<5$ spa repeats.

Previous studies have shown that MRSA isolates classified as communityassociated usually harbor either SCCmec type IV or V, and often PVL, but may differ in their genetic backgrounds (CC1, CC8, CC30, CC59 and CC80) [34]. In the 
EMR, $5 \%$ of the MRSA isolates were positive for PVL, which is higher than the previously reported $1.3 \%$ [2]. Thus, PVL-positive MRSA isolates with a heterogeneous genetic background are emerging in the EMR.

PVL-positive MRSA isolates associated with ST8-MRSA-IV, ST30-MRSA-IV, and ST80-MRSA-IV have been isolated in the Netherlands $[3,35]$. In the present study, 2 of the PVL-positive MRSA isolates harbored SCCmec type V. The different genetic background of these isolates, i.e., ST89 and ST772, a single-locus variant of ST1 at the pta locus, might suggest that SCCmec type $\mathrm{V}$ was introduced on different occasions into different S. aureus lineages. A PVL-positive ST772-MRSA$V$ has been observed in Germany [36]. One of the PVL-positive isolates harbored SCCmec type I, and such isolates with a ST30 and ST37 genetic background have been described in the Netherlands [3]. Although a recent study suggested that CNA and PVL combined contribute to virulence, only 6 of the 11 PVL-positive MRSA isolates from the EMR harbored the cna gene [37]. Further studies are needed to investigate the contribution of the combination of CNA and PVL to virulence.

The genetic background of 1 PVL-positive ST45-MRSA-IV isolate from Belgium was similar to that of the Berlin clone. Hitherto, only PVL-negative isolates with this background have been found in EMR countries [19, 20]. PVL-positive MRSA isolates, associated with the major CA-MRSA clones (ST8-MRSA-IV, ST30-MRSAIV, and ST80-MRSA-IV) have been reported from Belgium [38]. Because PVL is situated on a phage, the genes encoding PVL might have been transferred to $S$. aureus with a CC45 genetic background [34].

Our study found a PVL-positive MRSA isolate from Germany with spa type t042 (spa repeat pattern $\mathrm{r} 26 \mathrm{r} 23 \mathrm{r} 12 \mathrm{r} 34 \mathrm{r} 34 \mathrm{r} 33 \mathrm{r} 34)$. This spa type is strongly related to spa types t044 and t131 (spa repeat patterns r07r23r12r34r34r33r34 and r07r23r12r34r33r34, respectively), which are usually associated with the CAMRSA ST80-MRSA-IV clone found in Germany [34].

The cna gene has been previously observed among MRSA isolates from CC22, CC30, and CC45 $[23,29]$. Therefore, the presence of the cna gene might, together with spa typing, be used as a marker for different genetic backgrounds.

MRSA clones associated with the hospital associated-MRSA CCs 5, 8, 22, 30, and 45, the PVL-positive CA-MRSA CCS $1,8,30,80$, and 89, as well as MRSA related to pigs (ST389-MRSA-IV/V) were observed in the EMR. Dissemination of these clones is possible because of the introduction of new MRSA clones associated with travel; with patients who have previously been admitted to a hospital 
abroad (cross-border health care); or with other high-risk patients, such as pigfarmers and their families. Therefore, a cross-border search-and-contain policy may help control the further spread of MRSA and reduce the financial cost to hospitals, nursing homes, and the community in the EMR.

TABLE 4. Suggested cross-border dissemination of the major methicillin-resistant Staphylococcus aureus (MRSA) clones in the Euregio Meuse-Rhine region, July 2005-April 2006

\begin{tabular}{ll}
\hline MRSA clone & Previously observed in/possible dissemination from \\
\hline ST5-MRSA-II & Belgium,Germany \\
ST5-MRSA-IV & Belgium \\
ST8-MRSA-IV & Belgium, Germany, the Netherlands \\
ST22-MRSA-IV & Belgium, Germany \\
ST30-MRSA-IV & Belgium, Germany \\
ST36-MRSA-II & Belgium \\
ST45-MRSA-IV & Belgium, Germany, the Netherlands \\
\hline
\end{tabular}

MRSA: methicillin-resistant Staphylococcus aureus

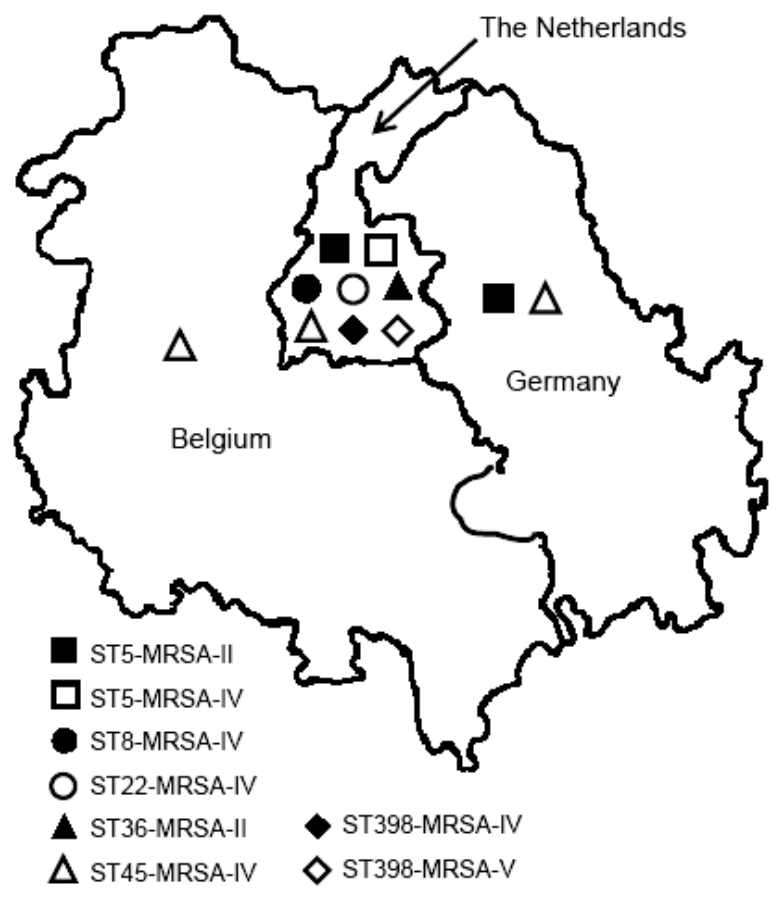

FIGURE 1. Distribution of the major methicillin-resistant Staphylococcus aureus (MRSA) clones in the Euregio Meuse-Rhine region, July 2005-April 2006 


\section{References}

1. Wolf $\mathrm{U}$, Hollederer A, Brand H. Cross-border cooperation in Europe: what are Euregios? Gesundheitswesen. 2006;68:667-73. DOI: 10.1055/s-2006-927265

2. Deurenberg RH, Vink C, Oudhuis GJ, Mooij JE, Driessen C, Coppens G, et al. Different clonal complexes of methicillin-resistant Staphylococcus aureus are disseminated in the Euregio MeuseRhine region. Antimicrob Agents Chemother. 2005;49:4263-71. DOI: 10.1128/AAC.49.10.42634271.2005

3. Wannet WJ, Spalburg E, Heck ME, Pluister GN, Tiemersma E, Willems RJ, et al. Emergence of virulent methicillin-resistant Staphylococcus aureus strains carrying Panton-Valentine leukocidin genes in the Netherlands. J Clin Microbiol. 2005;43:3341-5. DOI: 10.1128/JCM.43.7.33413345.2005

4. Clinical and Laboratory Standards Institute. Performance standards for antimicrobial susceptibility testing, Twelfth informational supplement, M100-S12. Wayne (PA): The Institute; 2002.

5. Toma E, Barriault D. Antimicrobial activity of fusidic acid and disk diffusion susceptibility testing criteria for gram-positive cocci. J Clin Microbiol. 1995;33:1712-5.

6. Fuchs PC, Jones RN, Barry AL. Interpretive criteria for disk diffusion susceptibility testing of mupirocin, a topical antibiotic. J Clin Microbiol. 1990;28:608-9.

7. O'Sullivan MV, Cai Y, Kong F, Zeng X, Gilbert GL. Influence of disk separation distance on accuracy of the disk approximation test for detection of inducible clindamycin resistance in Staphylococcus spp. J Clin Microbiol. 2006;44:4072-6. DOI: 10.1128/ JCM.01632-06

8. Oliveira DC, de Lencastre H. Multiplex PCR strategy for rapid identification of structural types and variants of the mec element in methicillin-resistant Staphylococcus aureus. Antimicrob Agents Chemother. 2002;46:2155-61. DOI: 10.1128/AAC.46.7.2155-2161.2002

9. Shore A, Rossney AS, Keane CT, Enright MC, Coleman DC. Seven novel variants of the staphylococcal chromosomal cassette mec in methicillin-resistant Staphylococcus aureus isolates from Ireland. Antimicrob Agents Chemother. 2005;49:2070-83. DOI: 10.1128/AAC.49.5.20702083.2005

10. Oliveira DC, Milheirico C, de Lencastre H. Redefining a structural variant of staphylococcal cassette chromosome mec, SCCmec type VI. Antimicrob Agents Chemother. 2006;50:3457-9. DOI: 10.1128/AAC.00629-06

11. Ito T, Ma XX, Takeuchi F, Okuma K, Yuzawa H, Hiramatsu K. Novel type V staphylococcal cassette chromosome mec driven by a novel cassette chromosome recombinase, ccrC. Antimicrob Agents Chemother. 2004;48:2637-51. DOI: 10.1128/AAC.48.7.2637-2651.2004

12. Zhang K, McClure JA, Elsayed S, Louie T, Conly JM. Novel multiplex PCR assay for characterisation and concomitant subtyping of staphylococcal cassette chromosome mec types I to $\mathrm{V}$ in methicillin-resistant Staphylococcus aureus. J Clin Microbiol. 2005;43:5026-33. DOI: 10.1128/JCM.43.10.5026-5033.2005

13. Nulens E, Stobberingh EE, van Dessel H, Sebastian S, van Tiel FH, Beisser PS, et al. Molecular characterisation of Staphylococcus aureus bloodstream isolates collected in a Dutch university hospital between 1999 and 2006. J Clin Microbiol. 2008;46:2438-41. DOI: 10.1128/JCM.00808-08 
14. Deurenberg RH, Vink C, Driessen C, Bes M, London N, Etienne J, et al. Rapid detection of PantonValentine leukocidin from clinical isolates of Staphylococcus aureus strains by real-time PCR. FEMS Microbiol Lett. 2004;240:225-8. DOI: 10.1016/j.femsle.2004.09.031

15. Deurenberg RH, Nieuwenhuis RF, Driessen C, London N, Stassen FR, van Tiel FH, et al. The prevalence of the Staphylococcus aureus tst gene among community- and hospital-acquired strains and isolates from Wegener's granulomatosis patients. FEMS Microbiol Lett. 2005;245:1859. DOI: 10.1016/j.femsle.2005.03.002

16. Amorim ML, Faria NA, Oliveira DC, Vasconcelos C, Cabeda JC, Mendes AC, et al. Changes in the clonal nature and antibiotic resistance profiles of methicillin-resistant Staphylococcus aureus isolates associated with spread of the EMRSA-15 clone in a tertiary care Portuguese hospital. J Clin Microbiol. 2007;45:2881-8. DOI: 10.1128/JCM.00603-07

17. Lindsay JA, Holden MT. Understanding the rise of the superbug: investigation of the evolution and genomic variation of Staphylococcus aureus. Funct Integr Genomics. 2006;6:186-201. DOI: 10.1007/s10142-005-0019-7

18. Wertheim HF, Melles DC, Vos MC, van Leeuwen W, van Belkum A, Verbrugh HA, et al. The role of nasal carriage in Staphylococcus aureus infections. Lancet Infect Dis. 2005;5:751-62. DOI: 10.1016/S1473-3099(05)70295-4

19. Deurenberg RH, Stobberingh EE. The evolution of Staphylococcus aureus. Infect Genet Evol. 2008;8:747-63. DOI: 10.1016/j. meegid.2008.07.007

20. Enright MC, Robinson DA, Randle G, Feil EJ, Grundmann H, Spratt BG. The evolutionary history of methicillin-resistant Staphylococcus aureus (MRSA). Proc Natl Acad Sci USA. 2002;99:7687-92. DOI: 10.1073/pnas.122108599

21. Hallin M, Denis O, Deplano A, De Mendonca R, De Ryck R, Rottiers S, et al. Genetic relatedness between methicillin-susceptible and methicillin-resistant Staphylococcus aureus: results of a national survey. J Antimicrob Chemother. 2007;59:465-72. DOI: 10.1093/jac/dkI535

22. Ferry $\mathrm{T}$, Bes M, Dauwalder O, Meugnier H, Lina G, Forey F, et al. Toxin gene content of the Lyon methicillin-resistant Staphylococcus aureus clone compared with that of other pandemic clones. $J$ Clin Microbiol. 2006;44:2642-4. DOI: 10.1128/JCM.00430-06

23. Diep BA, Carleton HA, Chang RF, Sensabaugh GF, Perdreau-Remington F. Roles of 34 virulence genes in the evolution of hospital- and community-associated strains of methicillin-resistant Staphylococcus aureus. J Infect Dis. 2006;193:1495-503. DOI: 10.1086/503777

24. Murchan S, Kaufmann ME, Deplano A, de Ryck R, Struelens M, Zinn CE, et al. Harmonization of pulsed-field gel electrophoresis protocols for epidemiological typing of strains of methicillinresistant Staphylococcus aureus: a single approach developed by consensus in 10 European laboratories and its application for tracing the spread of related strains. J Clin Microbiol. 2003;41:1574-85. DOI: 10.1128/JCM.41.4.1574-1585.2003

25. van Rijen MM, Van Keulen PH, Kluytmans JA. Increase in a Dutch hospital of methicillin-resistant Staphylococcus aureus related to animal farming. Clin Infect Dis. 2008;46:261-3. DOI: $10.1086 / 524672$

26. Wulf MW, Sorum M, van Nes A, Skov R, Melchers WJ, Klaassen $\mathrm{CH}$, et al. Prevalence of methicillin-resistant Staphylococcus aureus among veterinarians: an international study. Clin Microbiol Infect. 2008;14:29-34. DOI: 10.1111/j.1469-0691.2007.01873.x

27. Witte W, Strommenger B, Stanek C, Cuny C. Methicillin-resistant Staphylococcus aureus ST398 in humans and animals, Central Europe. Emerg Infect Dis. 2007;13:255-8. 
28. Durand G, Bes M, Meugnier H, Enright MC, Forey F, Liassine N, et al. Detection of new methicillinresistant Staphylococcus aureus clones containing the toxic shock syndrome toxin 1 gene responsible for hospital- and community-acquired infections in France. J Clin Microbiol. 2006;44:847-53. DOI: 10.1128/JCM.44.3.847-853.2006

29. Wielders $C L$, Vriens MR, Brisse S, de Graaf-Miltenburg LA, Troelstra A, Fleer A, et al. In-vivo transfer of mecA DNA to Staphylococcus aureus. Lancet. 2001;357:1674-5. DOI: 10.1016/S01406736(00)04832-7

30. Hanssen AM, Kjeldsen G, Sollid JU. Local variants of staphylococcal cassette chromosome mec in sporadic methicillin-resistant Staphylococcus aureus and methicillin-resistant coagulase-negative staphylococci: evidence of horizontal gene transfer? Antimicrob Agents Chemother. 2004;48:28596. DOI: 10.1128/AAC.48.1.285-296.2004

31. Chung M, Dickinson G, De Lencastre H, Tomasz A. International clones of methicillin-resistant Staphylococcus aureus in two hospitals in Miami, Florida. J Clin Microbiol. 2004;42:542-7. DOI: 10.1128/JCM.42.2.542-547.2004

32. Deplano A, De Mendonca R, De Ryck R, Struelens MJ. External quality assessment of molecular typing of Staphylococcus aureus isolates by a network of laboratories. J Clin Microbiol. 2006;44:3236-44. DOI: 10.1128/JCM.00789-06

33. Kondo Y, Ito T, Ma XX, Watanabe S, Kreiswirth BN, Etienne J, et al. Combination of multiplex PCRs for staphylococcal cassette chromosome mec type assignment: rapid identification system for $m e c, c c r$, and major differences in junkyard regions. Antimicrob Agents Chemother. 2007;51:26474. DOI: 10.1128/AAC.00165-06

34. Tristan A, Bes M, Meugnier H, Lina G, Bozdogan B, Courvalin P, et al. Global distribution of Panton-Valentine leukocidin-positive methicillin-resistant Staphylococcus aureus, 2006. Emerg Infect Dis. 2007;13:594-600.

35. Huijsdens XW, van Santen-Verheuvel MG, Spalburg E, Heck ME, Pluister GN, Eijkelkamp BA, et al. Multiple cases of familial transmission of community-acquired methicillin-resistant Staphylococcus aureus. J Clin Microbiol. 2006;44:2994-6. DOI: 10.1128/JCM.00846-06

36. Strommenger B, Braulke C, Pasemann B, Schmidt C, Witte W. Multiplex PCR for rapid detection of Staphylococcus aureus isolates suspected to represent community-acquired strains. J Clin Microbiol. 2008;46:582-7.

37. de Bentzmann S, Tristan A, Etienne J, Brousse N, Vandenesch F, Lina G. Staphylococcus aureus isolates associated with necrotizing pneumonia bind to basement membrane type I and IV collagens and laminin. J Infect Dis. 2004;190:1506-15. DOI: 10.1086/424521

38. Denis O, Deplano A, De Beenhouwer H, Hallin M, Huysmans G, Garrino MG, et al. Polyclonal emergence and importation of community-acquired methicillin-resistant Staphylococcus aureus strains harboring Panton-Valentine leukocidin genes in Belgium. J Antimicrob Chemother. 2005;56:1103-6. DOI: 10.1093/jac/dki379

39. Lindsay JA, Moore CE, Day NP, Peacock SJ, Witney AA, Stabler RA, et al. Microarrays reveal that each of the ten dominant lineages of Staphylococcus aureus has a unique combination of surfaceassociated and regulatory genes. J Bacteriol. 2006;188:669-76. DOI: 10.1128/JB.188.2.669676.2006 


\section{Chapter 6: Cost of the meticillin-resistant Staphylococcus aureus search and destroy policy in a Dutch university hospital}

E. Nulens, E. Broex, A. Ament, R.H. Deurenberg, E. Smeets, J. Scheres, F.H. van Tiel, B. Gordts, E.E. Stobberingh

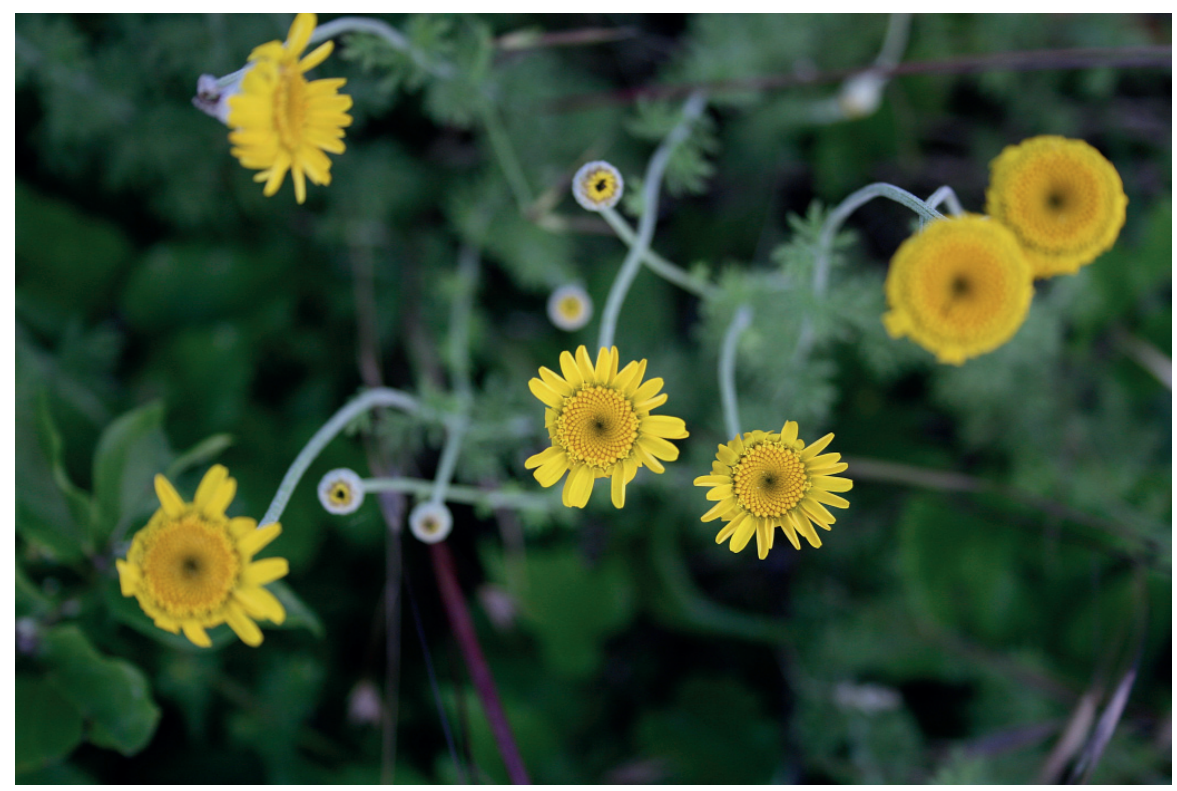

Yellow button, Lanaken, 2008

Published in

Journal of Hospital Infection. 2008;68,301-307.

(C) 2008 The Hospital Infection Society. Published by Elsevier Ltd. All rights reserved. 


\section{Summary}

Costs related to a search and destroy policy and treatment for Staphylococcus aureus bacteraemia in the University Hospital Maastricht were calculated for the period 2000 and 2004. The financial cost-benefit break-even point of the search and destroy policy was determined by modelling. On average 22,412 patients were admitted per year for an average of 8.7 days. Each year 246 patients were screened for meticillin-resistant Staphylococcus aureus (MRSA) and 74 patients were decolonised and nursed in preventive isolation. The prevalence of MRSA in the University Hospital Maastricht was $0.7 \%$, as calculated from positive blood cultures, and mean length of stay for all patients with $S$. aureus bloodstream infections was 39.9 days. The annual cost of pro-active searching for MRSA in the University Hospital Maastricht was $€ 1,383,200$, and $€ 2,738,128$ for MRSA prevention and treatment of $S$. aureus bloodstream infections. Simulation of a variety MRSA/meticillin-susceptible $S$. aureus (MSSA) ratios showed that even if the MRSA prevalence reaches $8 \%$, prevention costs are still lower than the cost of treating $S$. aureus infections. In conclusion, the total cost of a search and destroy policy is lower than the cost of treating $S$. aureus bloodstream infections in the University Hospital Maastricht. At a MRSA prevalence of $\leq 8 \%$ the search and destroy policy remains cost-effective. From an economic point of view, the search and destroy policy is the best alternative at maintaining an endemic MRSA level at $<1 \%$. 


\section{Introduction}

The Netherlands and the Scandinavian countries have implemented a successful search and destroy (S\&D) policy against meticillin-resistant Staphylococcus aureus (MRSA) that is associated with a low endemic level of MRSA in hospitals [1]. Patients and health care workers (HCWs) are actively screened for MRSA colonisation and/or infection, and high-risk patients are nursed in isolation until culture results exclude MRSA carriage. Little is known about the cost of this S\&D policy in The Netherlands, except for one publication by Vriens et al. [2]. The cost related to the S\&D policy is high, but may contribute to a lower cost of treatment for $S$. aureus bloodstream infection (BSI).

The aim of this study was to establish retrospectively the cost of the S\&D policy in the University Hospital Maastricht (UHM) and to determine the threshold of MRSA prevalence at which the S\&D policy would still be cost-effective ('breakeven point'). Furthermore, the cost of the S\&D policy and of treatment of BSI caused by meticillin-susceptible $S$. aureus (MSSA) and MRSA were compared in a model.

\section{Methods}

\section{Population}

All patients admitted to the UHM and presenting with multiple MRSA risk factors at the day of admission were screened for MRSA [1]. Patients are categorised into MRSA low- and high-risk groups, according to the estimated risk of MRSA colonisation. Patients admitted to a foreign hospital for at least $24 \mathrm{~h}$, in the preceding six months and with an additional risk factor for MRSA colonisation, e.g. recent operation, skin defects, intubation for mechanical ventilation, are considered as MRSA high-risk patients. In addition, patients with MRSA colonisation or infection in their history are considered as high risk. Patients admitted to a foreign hospital for at least $24 \mathrm{~h}$ in the preceding six months and without any other risk factors for MRSA colonisation present at the day of admission are considered as MRSA low-risk patients. Low-risk patients are only screened for MRSA carriage (Table 1). High-risk patients suspected to be colonised with MRSA are screened and nursed in preventive isolation until culture results are known. Patients colonised or unexpectedly found to be infected with MRSA are nursed in preventive isolation, decolonised and if necessary treated for infection. Once MRSA has been eradicated the isolation measures are lifted. 
HCWs and patients who are known to have been in contact with MRSA-positive patients are also screened for MRSA colonisation. HCWs who appear to have become MRSA positive are excluded from direct care of patients, decolonised with mupirocin and other measures, and return to patient care no sooner than screening cultures remain negative for MRSA. Patients are also decolonised, and isolation of the patient is stopped if no MRSA is found. If MRSA is isolated from a clinical sample of an unscreened patient, the patient is isolated and nursed in a single room, patients and HCWs are screened, and if other patients are colonised the ward will be closed.

\section{Study period and costs}

In a retrospective study relevant clinical data from patients admitted to the UHM between 2000 and 2004 were collected. All costs attributable to the S\&D policy as practiced were registered. Only costs spent in the hospital environment were taken into account, in order to define the financial consequences for the hospital. Costs due to loss of production by the hospital or personal costs for the patients were not included in the study. The number of patients, the length of stay (LOS) as well as the cost of hospitalisation for all patients admitted to the hospital were retrieved from the annual report of the financial and administrative department. The number of patients screened for MRSA, the number of MRSA surveillance cultures, the number of isolated patients and the total number of isolation days were registered by the infection control unit. Microbiological data were obtained from the medical microbiology department. The logistic department reported the cost of disposables. For every item in the S\&D policy the cost price as calculated by the financial department calculated was used. The total cost for a MRSA screening included the cost for microbiological culture, antibiotic susceptibility testing and detection of the mecA gene for all oxacillin- or cefoxitin-resistant $S$. aureus. MRSA carriers (patients and HCWs) were decolonised with mupirocin, along with other measures such as the use of antiseptic soap, changing linen and clothing every day, a strict personal hygiene code, and if necessary, systemic antibiotics.

The second part of the study consisted of collecting of the costs attributed for the treatment of $S$. aureus BSI. The data were collected from the same sources as described above.

Additional costs included the cost of an average of 14 days of flucloxacillin treatment for MSSA BSI, or vancomycin treatment for MRSA BSI, and serum level determination of vancomycin, whenever indicated. The LOS of the patients with S. aureus BSI was derived from the respective patient files. For all patients 
without BSI nursed in isolation the extra cost of isolation was calculated. This cost was estimated from the average LOS and the number of isolated and non-isolated patients and consisted of: (i) the cost for an isolation room; (ii) the cost of disposables, decolonisation of the patient, extra cleaning and disinfection of the patient room; and (iii) the cost for extra HCWs. The latter cost was formerly established during a MRSA outbreak in our hospital. During the outbreak, a separate group of HCWs was allocated to care only for the isolated patients. The time that HCWs nursed MRSA-positive patients, from entering the patient room, putting on a mask, gown and gloves until they left the patient room was measured with a chronometer by infection control nurses. By multiplying this nursing time with the cost per hour for HCWs, we derived the total cost for extra HCWs. This isolation cost also included the cost for screening HCWs.

For patients with a MRSA BSI, an additional isolation cost was taken into account, since these patients also have a longer hospital stay as compared to patients with MSSA BSI [3-6].

TABLE 1. Yearly average numbers of MRSA screenings of patients and S. aureus blood cultures at the University Hospital Maastricht

\begin{tabular}{|c|c|}
\hline \multicolumn{2}{|l|}{ General information } \\
\hline Number of admissions & 22,412 \\
\hline Length of stay & 8.7 days \\
\hline \multicolumn{2}{|l|}{ MRSA colonisation } \\
\hline Number of screenings & 246 \\
\hline Number of high-risk (isolated) patients & 70 \\
\hline Culture positive & 5 \\
\hline Culture negative & 65 \\
\hline Number of low-risk (non-isolated) patients & 176 \\
\hline Culture positive & 4 \\
\hline Culture negative & 172 \\
\hline Number of isolation days & 333 \\
\hline \multicolumn{2}{|l|}{ Blood cultures } \\
\hline Positive blood cultures & 59 \\
\hline Number of MRSA BSI & 0.4 \\
\hline Number of MSSA BSI & 58.6 \\
\hline Length of stay & 39.9 days \\
\hline
\end{tabular}

MRSA: methicillin-resistant Staphylococcus aureus MSSA: methicillin-susceptible Staphylococcus aureus $\mathrm{BSI}$ : bloodstream Infection 


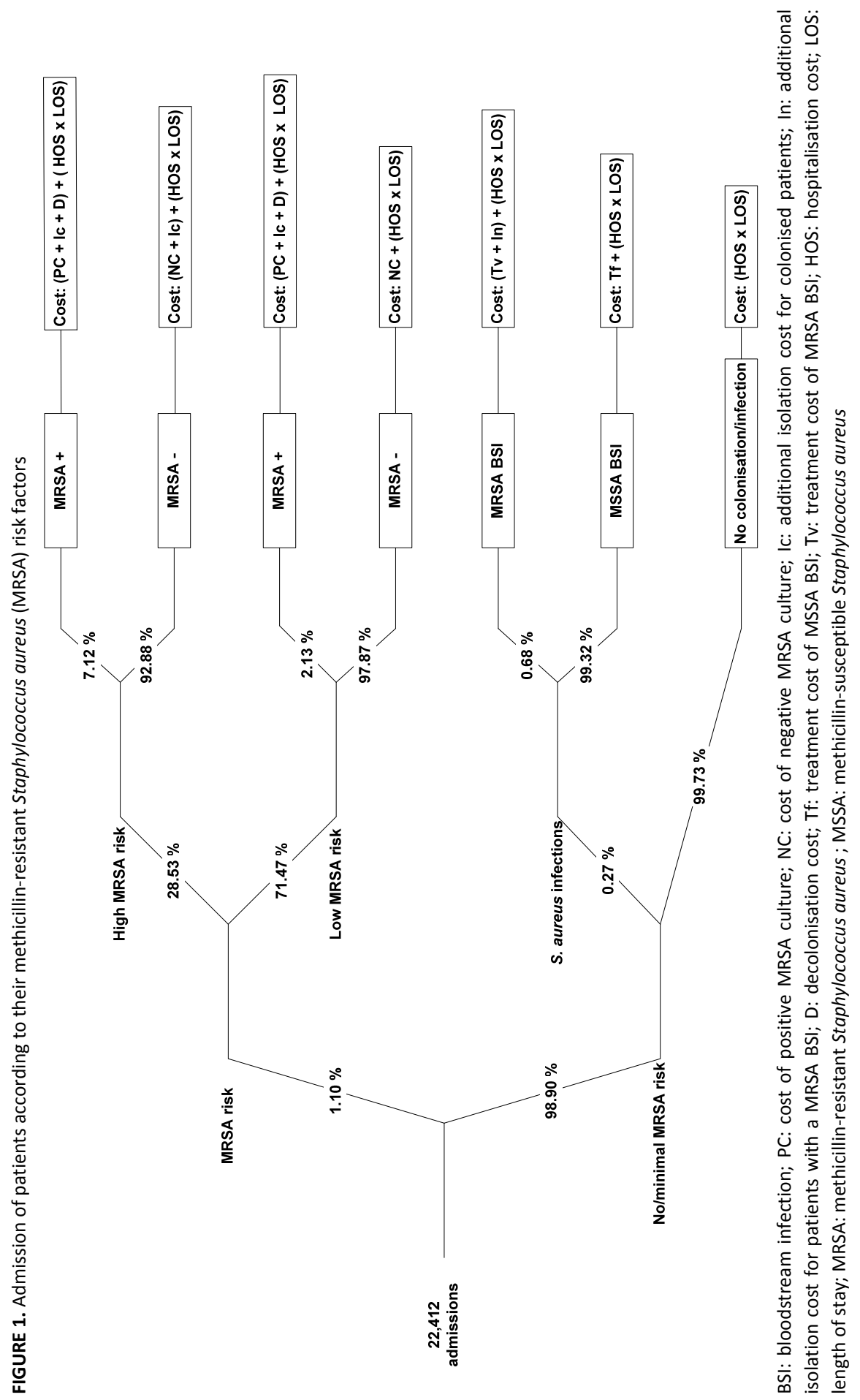




\section{Results}

The UHM is a 700 bed tertiary hospital, admitting on average 22,400 patients on a yearly basis (Table 1). In Figure 1, an algorithm for MRSA risk patients on admission is presented. An average of 246 patients per year was categorised as low or high MRSA colonisation risk. Yearly, five patients out of 70 in the high-risk group and four out of 176 in the low-risk group respectively carried MRSA $(P>0.05)$. MRSA carriers were nursed in isolation for a total of 333 days, or 4.5 isolation days per MRSA-positive patient on average. The costs related to each patient category are presented in Table 1 . An additional cost per patient of $€ 2,313$ (Ic) and $€ 6,372$ (In) was calculated for those nursed in isolation and with MRSA BSI, respectively (Figure 1).

Each year there was an average of 59 positive BSI S. aureus: 58.6 BSI caused by MSSA and 0.4 by MRSA. Hence the MRSA prevalence for $S$. aureus BSI in the UHM was $0.7 \%$. The average LOS for the general patient population was 8.9 days, but for patients with $S$. aureus BSI an average of 39.9 days was determined $(P<0.0001)$.

Table 2 presents the summary of cost of the S\&D policy. Patients colonised or infected with MRSA were isolated in a single room, at an additional cost of $€ 406$ a day, consisting of isolation measures (€24.26), extra HCWs (€289.32), disposables ( $€ 51.62$ per day) and additional room cleaning (€40.80). During the study, few patients developed a MRSA BSI, and an additional LOS of 10 days was assumed, representing a total additional cost of $€ 6,372$ per MRSA-infected patient.

The first row of Table 3 shows the basic cost of the S\&D policy and S. aureus BSI treatment. The total cost per year for the hospital stay for patients with a MRSA or MSSA BSI was $€ 29,315$ and $€ 24,047$ respectively. From these figures, the total cost of MRSA BSI was estimated for an increasing MRSA/MSSA infection ratio. The total cost for treating $S$. aureus BSI increased when the MRSA/MSSA ratio increased. At a MRSA/MSSA infection ratio of 10/90 and higher, the S. aureus infection treatment cost exceeded the S\&D policy cost. Therefore, the $8 \%$ prevalence is considered as the 'break-even point' (Figure 2).

\section{Discussion}

The University Hospital Maastricht is a 700 bed tertiary hospital, situated in the Euregion Meuse-Rhine, close to Belgian and German hospitals where MRSA is endemic [7]. So-called cross-border health care exists between hospitals in the region and the UHM, and patients arriving from foreign hospitals are always 
screened for MRSA upon hospital admission. The data presented in Figure 1 represent a small bias since they are based on the assumption that patients with S. aureus BSI always belong to the no- or minimal MRSA risk group (because a very limited number of MRSA BSI occurred) but in fact MSSA BSI may also occur in MRSA-colonised patients.

Pro-active MRSA screening and isolation of patients at risk are expensive, but so far this S\&D policy has contributed to the containment of the MRSA problem in hospitals in The Netherlands and Scandinavia. Simulations have shown that without preventive measures the prevalence of MRSA in a low MRSA prevalence hospital would steadily have increased through transmission of MRSA between patients and between HCWs and patients. On the other hand, isolating colonised patients and patients at high risk, as well as screening patients who have been in contact with an index case, maintains low endemicity [2, 8, 9]. Even in highprevalence hospitals this strategy will prevent transmission of MRSA, and selective MRSA screening of patients is as effective as a hospital-wide screening program, as well as being cheaper [2, 10-12].

In a large Dutch university hospital the yearly cost of the S\&D policy between 1991 and 2000 was estimated to be as high as $€ 280,000$ [2]. The present study demonstrates that the cost of the S\&D policy as applied in the UHM between 2000 and 2004 is in fact much higher. It is difficult to compare the differences in the cost between these two studies. The former study has been retrospectively conducted between 1991 and 2000. Even after correction of the cost with an average yearly inflation rate of $2.55 \%$ in The Netherlands in that period, the S\&D policy cost in the current study is higher [13]. Vriens et al. described a lower number of patients at risk for MRSA than in the current study, which is due to intensive cross-border health care exchange of patients between the UHM and neighbouring hospitals in Belgium and Germany. The cost of lost hospitalisation days and non-performed surgery was included in the former but not in the current study, whereas an extra cost of isolation was included in the total cost calculation for every isolated patient. The extra cost for isolating patients was calculated based on experience of a MRSA outbreak. By measuring the time an isolated MRSA colonised patient is nursed by HCWs, a more accurate estimate of the extra cost was determined.

Patients with a MSSA BSI were hospitalised significantly longer than the general patient population without $S$. aureus BSI. In this study the LOS, and therefore the cost for nursing patients with $S$. aureus BSI, is higher than in other studies; the reason for this remains unclear [6-10]. The determination of the extra LOS of MRSA BSI patients was not possible from our data, because on average fewer 
than one MRSA BSI occurred per year. An additional LOS of 10 days for MRSA BSI was estimated, based on the literature $[3,4,6]$. The connected cost was mainly due to the isolation measures. Hence the difference between the cost in nursing patients with MRSA and MSSA BSI is about $€ 6,086$ per patient. Referring to several other investigators using different calculation methods, the cost difference between MRSA- and MSSA-infected patients is estimated from \$2,500 to $\$ 3,700$ for a mixed group of infections to $\$ 6,900$ for MSSA and MRSA bacteraemia $[6,11,12,14]$.

TABLE 2. Yearly average cost of the methicillin-resistant Staphylococcus aureus (MRSA) search and destroy policy between 2000 and 2004 at the University Hospital Maastricht

\begin{tabular}{lr}
\hline & Cost $(\boldsymbol{€})$ \\
\hline Nursing & 570.80 \\
1 day, all patients & 976.80 \\
1 day, MRSA patients in isolation & \\
& \\
MRSA screening culture & 10.61 \\
Negative culture & 78.47 \\
Positive culture & \\
& \\
Treatment & 6.13 \\
Mupirocin ointment/carrier & 29.34 \\
Co-trimoxazole/carrier & 60.72 \\
Flucloxacillin/MSSA BSI & 250.13 \\
Vancomycin/MRSA BSI & \\
& 20.00 \\
Vancomycin dosage & $1,383,200.00$ \\
\hline
\end{tabular}

MRSA: methicillin-resistant Staphylococcus aureus MSSA: methicillin-susceptible Staphylococcus aureus BSI: bloodstream Infections

S\&D: search and destroy

A drawback to our study is that the increasing cost of $S$. aureus BSI (Figure 2) is always compared with the unchanged cost of the S\&D policy. When the MRSA prevalence increases, more patients would have to be isolated, hence more patients and HCWs would have to be screened and isolated, and the cost of the S\&D strategy would increase. Nevertheless, a higher MRSA prevalence would result in more MRSA infections and the treatment cost would also rise substantially. 
In this study, we assumed that MRSA BSI substitutes for MSSA BSI; i.e. that the MRSA/MSSA proportion might change but the absolute number of $S$. aureus BSI does not. However, at least some other studies contradict this assumption [12, $14]$. In that case, the cost for $S$. aureus BSI treatment would not only increase due to the increase of MRSA BSI but also due to the increase of the absolute number of $S$. aureus BSI. More patients would have to be treated with substantialy more attributable morbidity and mortality.

The cost of mortality is often neglected in cost studies. For this reason the cost of MRSA infections may be underestimated when patients die sooner, thus reducing the costly hospitalisation. When the cost of a human life is not taken into account, only the increased morbidity due to MRSA, but not the increased mortality, adds to the hospital cost $[6,14]$. MRSA mortality is difficult to estimate, but if this cost is included the total MRSA-related cost would be substantially higher $[14,15]$.

TABLE 3. Variations in the $S$. aureus treatment costs with changing MRSA/MSSA infection ratio

\begin{tabular}{lcrrrr}
\hline \multirow{2}{*}{$\begin{array}{l}\text { MRSA/MSSA } \\
\text { ratio }\end{array}$} & \multirow{2}{*}{ MRSA BSI/year } & \multicolumn{4}{c}{ Cost (€) } \\
\cline { 3 - 6 } & & MRSA BSI & MSSA BSI & S. aureus BSI & Total MRSA \\
\hline $0.7 / 99.3$ & 0.4 & 11,762 & $1,343,166$ & $1,354,928$ & $2,738,128$ \\
$2 / 98$ & 1.2 & 34,815 & $1,325,258$ & $1,360,072$ & $2,743,272$ \\
$5 / 95$ & 3.0 & 87,037 & $1,284,688$ & $1,371,725$ & $2,754,925$ \\
$8 / 92$ & 4.7 & 139,259 & $1,244,119$ & $1,383,378$ & $2,766,578$ \\
$10 / 90$ & 5.9 & 174,074 & $1,217,073$ & $1,391,147$ & $2,774,347$ \\
$25 / 75$ & 14.8 & 435,184 & $1,014,228$ & $1,449,412$ & $2,832,612$ \\
$50 / 50$ & 29.6 & 870,368 & 676,152 & $1,546,520$ & $2,929,720$ \\
\hline
\end{tabular}

MRSA: methicillin-resistant Staphylococcus aureus MSSA: methicillin-susceptible Staphylococcus aureus BSI: bloodstream Infections

In conclusion, the UHM spent $€ 1,383,200$ annually for MRSA pro-active preventive measures and this policy has kept the MRSA prevalence below $1 \%$. Therefore, the S\&D policy as applied in the UHM is still cost-effective and seems to be so far the best alternative. Even if the MRSA prevalence increased to $8 \%$, the S\&D policy would still be preferable in terms of costs. 


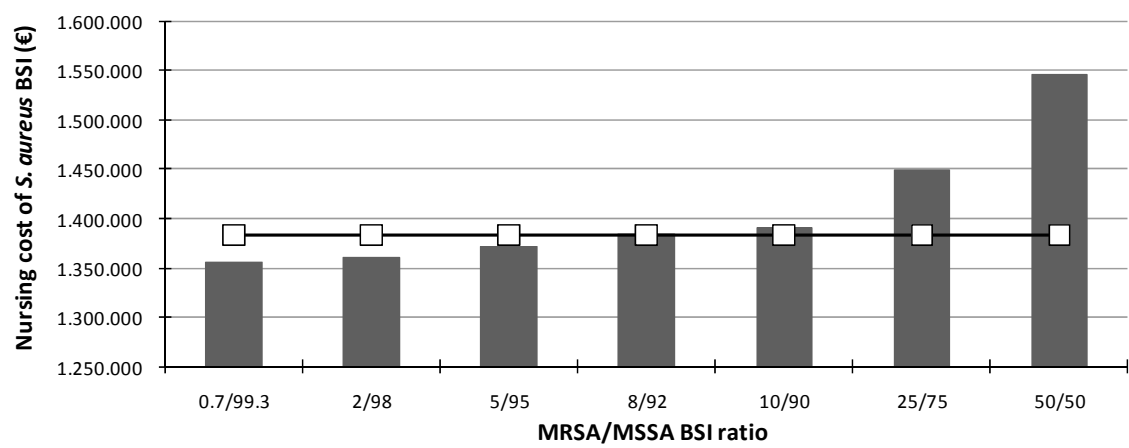

FIGURE 2. Comparison of the cost of the search and destroy policy in the University Hospital Maastricht with the $S$. aureus bloodstream infection (BSI) nursing cost and a changing MRSA/MSSA ratio 


\section{References}

1. Werkgroep Infectie Preventie. Richtlijn MRSA ziekenhuis, januari 2007.

2. Vriens $\mathrm{M}$, Blok $\mathrm{H}$, Fluit $\mathrm{A}$, et al. Costs associated with a strict policy to eradicate methicillinresistant Staphylococcus aureus in a Dutch university medical center: a 10 year survey. Eur J Clin Microbiol Infect Dis. 2002;21:782-786.

3. Kunori T, Cookson B, Roberts JA, Stone S, Kibbler C. Cost-effectiveness of different MRSA screening methods. J Hosp Infect. 2002;51:189-200.

4. Kaye KS, Engemann JJ, Mozaffari E, Carmeli Y. Reference group choice and antibiotic resistance outcomes. Emerg Infect Dis. 2004;10:1125-1128.

5. Rubin RJ, Harrington CA, Poon A, Dietrich K, Greene JA, Moiduddin A. The economic impact of Staphylococcus aureus infection in New York hospitals. Emerg Infect Dis. 1999;5:9-17.

6. Abramson MA, Sexton DJ. Nosocomial methicillin-resistant and methicillin-susceptible Staphylococcus aureus primary bacteremia: at what costs? Infect Control Hosp Epidemiol. 1999;20:408-411.

7. Tiemersma EW, Bronzwaer SLAM, Lyytikäinen O, et al. Methicillin-resistant Staphylococcus aureus in Europe, 1999-2002. Emerg Infect Dis. 2004;10:1627-1634.

8. Cooper BS, Stone SP, Kibbler CC, et al. Systematic review of isolation policies in the hospital management of methicillin-resistant Staphylococcus aureus: a review of the literature with epidemiological and economic modelling. Health Technol Assess. 2003;7:1-194.

9. Bootsma MCJ, Diekmann O, Bonten MJM. Controlling methicillin-resistant Staphylococcus aureus: quantifying the effects of interventions and rapid diagnostic testing. Proc Natl Acad Sci USA. 2006;103:5620-5625.

10. Wernitz MH, Keck S, Swidsinski S, Schulz S, Veit SK. Cost analysis of a hospital-wide selective screening programme for methicillin-resistant Staphylococcus aureus (MRSA) carriers in the context of diagnosis related groups. Clin Microbiol Infect. 2005;11:466-471.

11. Karchmer TB, Durbin $L$, Simonton BM, Farr BM. Cost-effectiveness of an active surveillance culture and contact/droplet precautions for control of methicillin-resistant Staphylococcus aureus. J Hosp Infect. 2002;51:126-132.

12. Chaix C, Durand-Zaleski I, Alberti C, Brun-Buisson C. Control of endemic methicillin-resistant Staphylococcus aureus. A cost benefit analysis in an intensive care unit. J Am Med Assoc. 1999;282:1745-1751.

13. Centraal Bureau voor de Statistiek. CBS Persbericht PB07-084, 2007.

14. Gould IM. Costs of hospital-acquired methicillin-resistant Staphylococcus aureus (MRSA) and its control. Int J Antimicrob Agents. 2006;28:379-384.

15. Report: deaths involving MRSA: England and Wales, 2000-2004. Health Stat Q. 2006;29:63-68. 


\section{Chapter 7: Contribution of two molecular assays as compared to selective culture for MRSA screening in a low MRSA prevalence population}

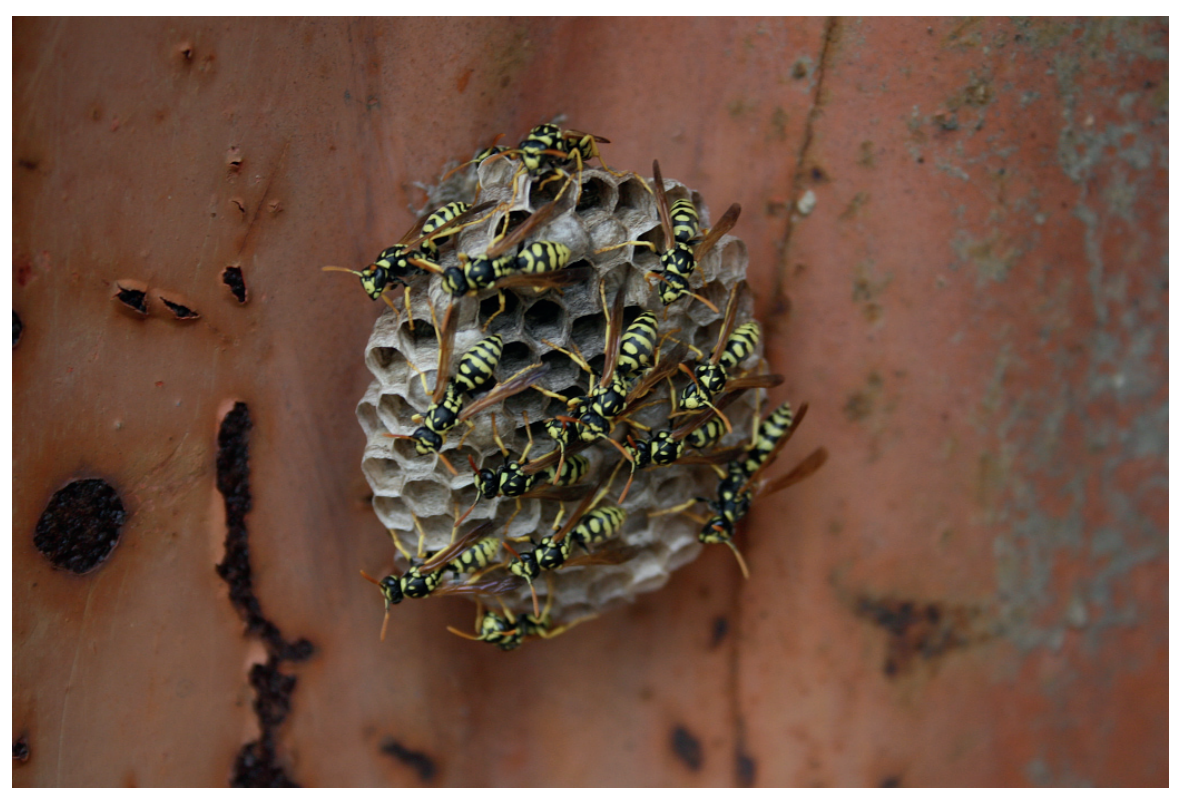

Wasps, Firenze, 2009

Published in

Infections. 2010;38:98-101.

(C) Urban \& Vogel 


\section{Abstract}

\section{Background}

As the prompt detection of methicillin-resistant Staphylococcus aureus (MRSA) carriers upon admission is fundamental in the MRSA prevention strategy of our hospital, the infection control team is eagerly seeking the most sensitive and rapid screening method. The aim of this study was to compare the performance of two molecular techniques with a conventional MRSA-selective culture test (Bio-Rad chromogenic MRSASelect) in order to elucidate the suitability of the assays specifically in an expected low MRSA prevalence population.

\section{Patients and Methods}

The anterior nares and throat of 500 patients and visitors attending the emergency department of Sint-Jan General Hospital between May and June 2007 were sampled, and MRSA carriage was determined by selective culture after enrichment and the BD GeneOhm ${ }^{\mathrm{TM}}$ StaphSR and the Cepheid Xpert ${ }^{\mathrm{TM}}$ MRSA assays.

\section{Results}

Eight MRSA carriers were detected by selective culture (1.6\% prevalence). The sensitivity, specificity, negative predictive value, and positive predictive value were 62.5, 99.0, 99.4, and 50.0\% for BD GeneOhm ${ }^{\mathrm{TM}}$ StaphSR and 62.5, 97.7, 99.4, and 31.3\% for Cepheid Xpert ${ }^{\mathrm{TM}}$ MRSA, respectively.

\section{Conclusions}

We conclude that MRSA rapid screening techniques must be interpreted cautiously in a low-prevalence population, as the sensitivity is lower than in selected high-risk populations. MRSA carriers detected with molecular techniques must be confirmed by conventional culture methods for follow-up. The specificity and negative predictive value indicate that molecular rapid methods are worthwhile to be considered in MRSA-preventive strategies. 


\section{Introduction}

The early detection of methicillin-resistant Staphylococcus aureus (MRSA) among hospital patients is essential in preventing its spread. Selective culture is often used as a screening method, but may take up to 5 days. Other, more rapid assays have been developed; some are still experimental and their performance unevaluated, while others are already Food and Drug Administration (FDA)approved [1]. On the other hand, studies indicating the usefulness of these rapid methods are carried out in patient populations with a high MRSA prevalence, or their performance are concluded from their ability to detect MRSA directly on bacterial isolates [2]. The aim of the present study was to evaluate the performance of two molecular assays, the BD GeneOhm ${ }^{\mathrm{TM}}$ StaphSR assay (Becton \& Dickinson, Franklin Lakes, NJ, USA) and the Cepheid Xpert ${ }^{\mathrm{TM}}$ MRSA assay (Cepheid, Sunnyvale, CA, USA), and their usefulness for the detection of MRSA in an assumed low MRSA prevalence population.

\section{Materials and Methods}

Between May and June 2007, 500 visitors (patients and their companions) of the emergency care department of the Sint-Jan General Hospital in Brugge, Belgium, were screened for MRSA. The infection control nurses informed all possible participants and collected the informed consent required from all volunteers. The infection control nurses performed the sampling for culture and the molecular assays.

Culture was always performed as the first screening method with one cotton swab with Amies transport medium (Copan, Brescia, Italy) at the throat and the anterior nares.

In half of the volunteers, which were randomly selected, the second sample was used for the GeneOhm ${ }^{\mathrm{TM}}$ StaphSR assay and the third sample for the Cepheid Xpert $^{\mathrm{TM}}$ MRSA assay. For the other 250 patients, the sampling order for the molecular assays was inversed. The sampling order of the molecular assays was printed on the informed consent sheet. Sampling was performed at the nose according to the instructions of the manufacturer with one cotton swab with Amies transport medium (Copan, Brescia, Italy) and with paired swabs (Venturi Transystem, Copan, Brescia, Italy) with liquid Stuart transport medium for the GeneOhm ${ }^{\mathrm{TM}}$ StaphSR and Cepheid Xpert ${ }^{\mathrm{TM}}$ MRSA assays, respectively.

The swabs were marked according to the study protocol and placed in a plastic bag together with the informed consent. The complete set of samples were 
stored in the refrigerator and transported twice a day to and were processed by the Medical Microbiology laboratory.

\section{Culture}

Upon arrival in the laboratory, the swab for culture was immediately suspended in Tryptic Soy Broth supplemented with $3.5 \% \mathrm{NaCl}$, incubated overnight at $35^{\circ} \mathrm{C}$, and inoculated on MRSASelect agar (Bio-Rad, Hercules, CA, USA) and Columbia blood agar, and incubated at $35^{\circ} \mathrm{C}$ for $48 \mathrm{~h}$. The culture plates were inspected daily for the development of MRSA and $S$. aureus, respectively. Pink colonies on MRSASelect agar and morphology consistent with $S$. aureus on Columbia blood agar were further identified. The identification of $S$. aureus was confirmed using Staphaurex (Remel, Lenexa, KS, USA) and resistance to cefoxitin and oxacillin was determined using the Clinical Laboratory Standards Institute (CLSI) disk diffusion method [3]. Several colonies were picked up from all $S$. aureus isolates on Columbia blood agar and MRSASelect agar, and kept at $-20^{\circ} \mathrm{C}$.

\section{Molecular Assays}

\section{GeneOhm $^{\mathrm{TM}}$ StaphSR Assay}

The swab was broken off into a microfuge tube containing sample buffer and vortexed for $1 \mathrm{~min}$. The entire cell suspension was transferred in a lysis buffer and the specimen was processed according to the manufacturer's instructions: $3 \mu \mathrm{l}$ of lysate was added to $25 \mu \mathrm{l}$ of master mix and real-time polymerase chain reaction (PCR) was performed using the Smart $\mathrm{Cycler}^{\mathrm{TM}}$ instrument. All lysates were kept at $-20^{\circ} \mathrm{C}$ until testing was complete. In every run of 6 or 14 swabs, a positive and a negative control were included. All assays were performed within $24 \mathrm{~h}$ after reception. In case of an invalid result, retesting was not performed.

\section{Cepheid Xpert ${ }^{\mathrm{TM}}$ MRSA}

At the laboratory, one of the paired swabs was suspended and broken off into a vial with elution buffer and vortexed for $10 \mathrm{~s}$. The entire cell suspension was then transferred to a sample cartridge and testing was performed according to the instructions of the manufacturer. Any specimen that gave an invalid or no result by the system was retested with the second swab as recommended by the manufacturer. All assays were performed on the same day of reception. All cartridges were discarded once the tests were completed. 


\section{Discordant Results}

All isolates from discrepant results were further examined. Isolates were identified as $S$. aureus with Staphaurex (Remel, Lenexa, KS, USA). PBP2' agglutination (Oxoid, Hampshire, United Kingdom) and culture on Mueller-Hinton agar with $6 \mu \mathrm{g} / \mathrm{ml}$ oxacillin and CHROMagar MRSA plates (Becton \& Dickinson, Franklin Lakes, NJ, USA) was performed to confirm oxacillin-resistance phenotypically. The presence of a $S$. aureus specific gene and the MRSA-specific mecA gene was performed according to the protocol of Martineau et al. [4].

Table 1. Comparison of two molecular methicillin-resistant Staphylococcus aureus (MRSA) detection assays with conventional enrichment and culture of MRSA

\begin{tabular}{|c|c|c|c|c|c|}
\hline & & \multicolumn{4}{|c|}{ MRSA detection } \\
\hline & & \multicolumn{2}{|c|}{ BD GeneOhm ${ }^{\mathrm{TM}}$ StaphSR } & \multicolumn{2}{|c|}{ Cepheid Xpert ${ }^{\mathrm{TM}}$ MRSA } \\
\hline & & + & - & + & - \\
\hline \multirow[t]{2}{*}{ MRSA Culture } & + & 5 & 3 & 5 & 3 \\
\hline & - & 5 & 485 & 11 & 477 \\
\hline Sensitivity (\%) & & 62.5 & & 62.5 & \\
\hline Specificity (\%) & & 99.0 & & 97.7 & \\
\hline PPV (\%) & & 50.0 & & 31.3 & \\
\hline NPV (\%) & & 99.4 & & 99.4 & \\
\hline
\end{tabular}

Two and four samples with an invalid molecular result for BD GeneOhm ${ }^{\mathrm{TM}}$ StaphSR and Cepheid Xpert $^{\mathrm{TM}}$ MSRA, respectively, were not included in the table.

PPV: positive predictive value

NPV: negative predictive value

\section{Results}

Non-selective culture and selective culture after enrichment detected 186 (37.2\%) S. aureus carriers and MRSA in eight (1.6\%) of the 500 volunteers. MRSA was detected in ten with the GeneOhm ${ }^{\text {TM }}$ StaphSR assay and 16 samples with the Cepheid Xpert ${ }^{\mathrm{TM}}$ MRSA assay. The Cepheid Xpert ${ }^{\mathrm{TM}}$ MRSA assay and GeneOhm ${ }^{\mathrm{TM}}$ StaphSR assay gave a positive signal in 11 and five samples, respectively, which were not confirmed with culture on MRSASelect agar. On the other hand, both assays failed to detect three confirmed MRSA cases detected by the selective culture (Table 1).

The first screening swab of the Cepheid Xpert $^{\mathrm{TM}}$ MRSA assay yielded an invalid or no result in 25 cases (5\%). After testing of the second swab, all except four of them were negative $(0.8 \%)$. For two samples, an invalid result was obtained with the GeneOhm ${ }^{\mathrm{TM}}$ StaphSR assay. It was not possible to repeat the assay of the 
samples because only one swab per patient was taken. These six invalid results were not included in Table 1.

Discordant results between culture and at least one molecular assay were further examined (Table 2). In three samples, MRSA was cultured, which was confirmed by antibiotic susceptibility and PBP2' testing, and amplification of the mecA gene, although MRSA was not detected either of the assays.

In seven samples, MSSA was cultured, whereas MRSA detection with both assays gave variable results (Table 2). Isolates failed to grow on Mueller-Hinton agar plates with oxacillin and all were tested to be PBP2'-negative.

In five samples, S. aureus was not cultured, although at least one assay gave a MRSA-positive signal.

\section{Discussion}

Sint-Jan General Hospital in Brugge, Belgium, is a tertiary 950-bed hospital, and an average of 26,495 patients per year are admitted. MRSA detection with culture is the standard procedure. However, this technique is laborious and slow. Rapid molecular and non-molecular MRSA assays are used or are being developed to overcome this delay [1].

The presence of MRSA in clinical samples with GeneOhm ${ }^{\mathrm{TM}}$ StaphSR and Cepheid Xpert $^{\mathrm{TM}}$ MRSA may be demonstrated by the real-time amplification of a single amplicon of the right extremity sequences of the staphylococcal cassette chromosome mec ( $\mathrm{SCCmec}$ ) and of orfX. Currently, seven major SSCmec types have been described, and both assays are able to detect $\mathrm{SCCmec}$ types $\mathrm{I}-\mathrm{V}$, but variants of SCCmec type IV and V, new SCCmec, or non-typable SCCmec elements may be missed $[1,5-8]$. In this study, three MRSA isolates were not detected with both assays, possibly due to them being SCCmec types that are non-detectable by both assays.

On the other hand, MSSA isolates that gave a false-positive signal with rapid MRSA assays have been reported. We cultured seven MSSA isolates from samples with false-positive reactions in at least one molecular assay. Further DNA investigation of the isolates did not reveal the presence of the mecA gene. An explanation for this could be that partial deletions in the mecA region and SCCmec sequences can occur, while the $3^{\prime}$ end of the $\mathrm{SCCmec}$ may still be present and may, therefore, be amplified $[5,9,10]$. 
In five samples of this study, a positive result with at least one molecular assay was not confirmed with culture. Molecular assays may be more sensitive than culture - up to 50 colony-forming units (CFU) are detected - while culture and enrichment detect up to $100 \mathrm{CFU}$ per $\mathrm{ml}$ [1]. On the other hand, in three cultureand mecA-confirmed MRSA isolates from three samples, neither molecular assay detected MRSA. The hypothesis is that the concentration of MRSA in the samples is lower than the limit of detection of the assays. The application of different swabs during the study may have influenced the outcome of the results.

Invalid or no results occurred for up to $5 \%$ of specimens tested with Cepheid Xpert $^{\mathrm{TM}}$ MRSA. With the use of the second swab, the number of invalid decreased to $0.8 \%$, but at increased cost. In a recent publication, no invalid results were obtained if samples were taken with eSwab ${ }^{\oplus}[11]$.

Table 2. Discordant results between culture and the two molecular assays

\begin{tabular}{|c|c|c|c|c|}
\hline Sample ID & Culture & BD GeneOhm ${ }^{\text {TM }}$ StaphSR & Cepheid Xpert $^{\mathrm{TM}}$ MRSA & mecA \\
\hline 71 & - & - & + & NT \\
\hline 96 & - & - & + & NT \\
\hline 124 & - & + & + & NT \\
\hline 314 & - & - & + & NT \\
\hline 466 & - & - & + & NT \\
\hline 99 & MSSA & + & - & - \\
\hline 100 & MSSA & + & + & - \\
\hline 146 & MSSA & - & + & - \\
\hline 222 & MSSA & + & + & - \\
\hline 346 & MSSA & + & + & - \\
\hline 374 & MSSA & - & + & - \\
\hline 482 & MSSA & - & + & - \\
\hline 30 & MRSA & - & - & + \\
\hline 285 & MRSA & - & - & + \\
\hline 330 & MRSA & - & - & + \\
\hline
\end{tabular}

MRSA: methicillin-resistant Staphylococcus aureus

MSSA: methicillin-susceptible Staphylococcus aureus

- : negative test result

+ : positive test result

NT: not tested

The BD GeneOhm ${ }^{\text {TM }}$ MRSA, an analogous earlier version of the GeneOhm ${ }^{\mathrm{TM}}$ StaphSR assay, has been extensively evaluated [1, 2]. The sensitivity and specificity in these studies were between 81 and $100 \%$ and between 61 and 99\%, respectively. The positive predictive values were between 56 and $98 \%$ and the 
negative predictive values were greater than 90\%. Most of these studies were performed in selected populations with a high MRSA prevalence.

In this setting, patients with a negative test result may be reliably be considered as non-carriers. However, in hospitals with a low MRSA prevalence and, therefore, a low pre-test probability, the positive predictive value of both molecular assays is apparently too low to reliably detect MRSA carriers, and, consequently, patients with a positive result may be unnecessarily isolated at an increased isolation cost.

\section{Acknowledgment}

We wish to thank Becton \& Dickinson for their technical support. 


\section{References}

1. Malhotra-Kumar S, Haccuria K, Michiels M, leven M, Poyart C, Hryniewicz W, Goossens H. Current trends in rapid diagnostics for methicillin-resistant Staphylococcus aureus and glycopeptideresistant Enterococcus species. J Clin Microbiol. 2008;46:1577-1587.

2. Deurenberg RH, Vink C, Sebastian S, Wassenberg MWM, Stobberingh EE. Directe en snelle detectie van meticillineresistente Staphylococcus aureus (MRSA). NTMM. 2007;15:97-103. Available online at: http://www.nvmm.nl/nvmm/nvmmcms.nsf/uploads/99CE5BC2151216 DDC12573C3006C82C9/\$FILE/NTMM-2007-3.pdf

3. Clinical Laboratory Standards Institute (CLSI). Performance standards for antimicrobial disk susceptibility tests, approved standard, 9th edn. CLSI, Wayne, PA, 2006.

4. Martineau F, Picard FJ, Lansac N, Ménard C, Roy PH, Ouellette M, Bergeron MG. Correlation between the resistance genotype determined by multiplex PCR assays and the antibiotic susceptibility patterns of Staphylococcus aureus and Staphylococcus epidermidis. Antimicrob Agents Chemother. 2000;44:231-238.

5. Huletsky A, Giroux R, Rossbach V, Gagnon M, Vaillancourt M, Bernier M, Gagnon F, Truchon K, Bastien M, Picard FJ, van Belkum A, Ouellette M, Roy PH, Bergeron MG. New real-time PCR assay for rapid detection of methicillin-resistant Staphylococcus aureus directly from specimens containing a mixture of staphylococci. J Clin Microbiol. 2004;42:1875-1884.

6. de San N, Denis O, Gasasira MF, De Mendonça R, Nonhoff C, Struelens MJ. Controlled evaluation of the IDI-MRSA assay for detection of colonisation by methicillin-resistant Staphylococcus aureus in diverse mucocutaneous specimens. J Clin Microbiol. 2007;45:1098-1101.

7. Deurenberg RH, Vink C, Kalenic S, Friedrich AW, Bruggeman CA, Stobberingh EE. The molecular evolution of methicillin-resistant Staphylococcus aureus. Clin Microbiol Infect. 2007;13:222-235.

8. Deurenberg RH, Stobberingh EE. The evolution of Staphylococcus aureus. Infect Genet Evol. 2008;8:747-763.

9. Donnio P-Y, Février F, Bifani P, Dehem M, Kervégant C, Wilhelm N, Gautier-Lerestif A-L, Lafforgue $N$, Cormier M, MR-MSSA Study Group of the Collège de Bactériologie-Virologie-Hygiène des Hôpitaux de France, Le Coustumier A. Molecular and epidemiological evidence for spread of multiresistant methicillin-susceptible Staphylococcus aureus strains in hospitals. Antimicrob Agents Chemother. 2007;51:4342-4350.

10. Rupp J, Fenner I, Solbach W, Gieffers J. Be aware of the possibility of false-positive results in single-locus PCR assays for methicillin-resistant Staphylococcus aureus. I Clin Microbio. 2006;44:2317.

11. Martens K, De Beenhouwer H, Frans J, Van den Abeele A, Cartuyvels R, Coppens G, on Behalf of the Bilulu Study Group. Evaluation of eSwab for surveillance of MRSA by Xpert MRSA and culture on pooled samples. $19^{\text {th }}$ European Congress of Clinical Microbiology and Infectious Diseases, Helsinki, Finland, May 2009, abstract no. P797. 



\section{Part 3: DISCUSSION}

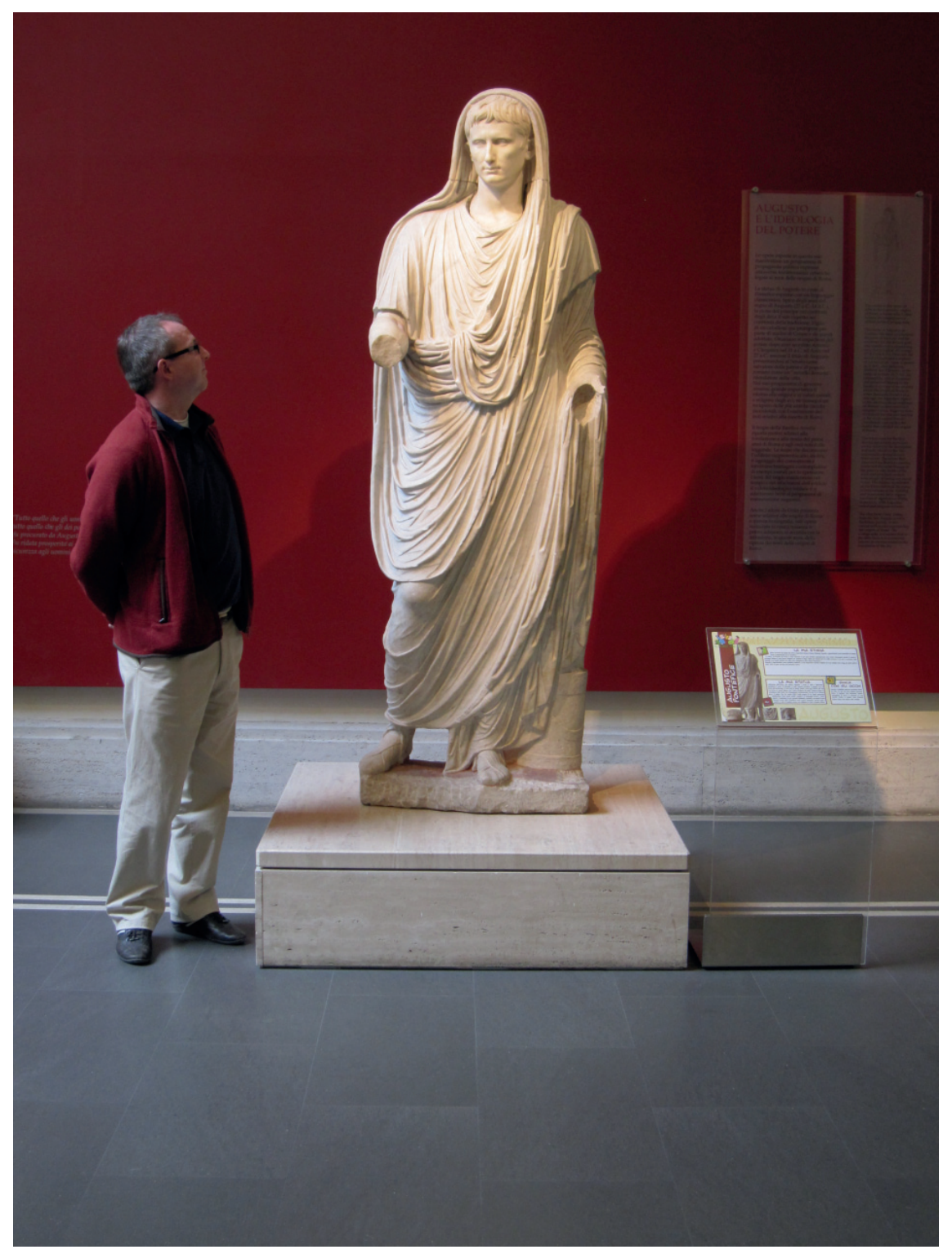

August Emperor, Museo Nationale Romano, Rome, 2010 



\section{Chapter 8: Discussion}

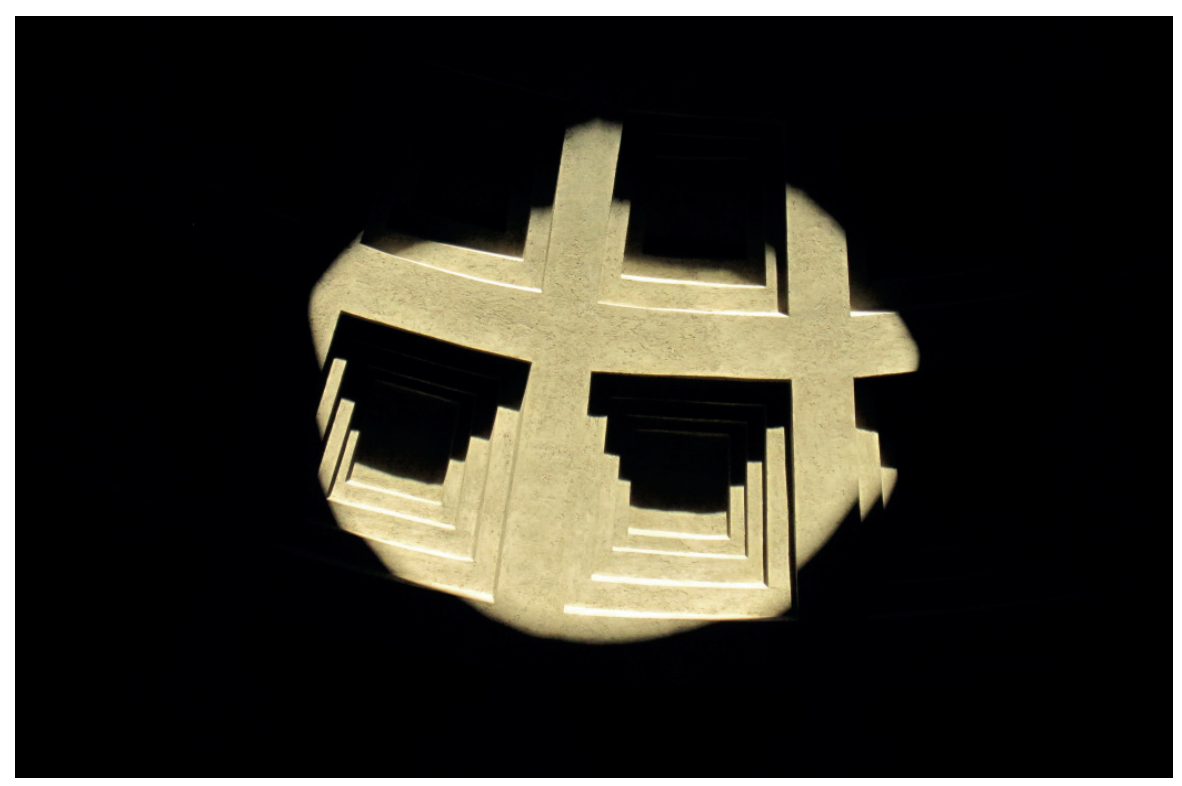

Sunbeam projected on the dome of the Pantheon, Rome, 2010 


\section{Genetic structure of the MSSA population (1999-2006)}

To get an overview of the genetic background of MSSA, isolates from blood cultures in the period 1999-2006 were typed by real-time amplification and sequencing of the spa locus. All spa types were clustered into spa-CC using the BURP algorithm with Ridom StaphType. The associated MLST CCs were allocated through the SpaServer. Up to $50 \%$ of the MSSA had a genetic background as observed in epidemic HA-MRSA clones, i.e., CC5, CC8, CC22, CC30, or CC45, and CA-MRSA clones, i.e., CC1, CC8, CC30, or CC59. These MSSA clones have also been described in isolates from other European countries and in other parts of the world, both in recent isolates and in isolates from the 60s. This finding suggests that these MSSA clones are ubiquitous and that they may provide a stable genetic background for acquiring SCCmec. Other MSSA clones from this study that have not been associated with MRSA were also found in other countries, even in low MRSA prevalence hospitals. The fact that more MSSA CCs were found compared with MRSA CCs, suggest that MSSA is highly clonal, and genetic more heterogeneous than MRSA. Only a few MSSA clones seem to offer a stable genetic environment to become MRSA. spa-CC012 was the main spa type in this population (43\%), and consisted of several MLST CCs. The heterogeneity of spaCCO12 may be explained by large chromosomal replacement including the spa locus, between different $S$. aureus lineages.

However, it is not clear yet what defines a stable genetic environment. Several intrinsic and extrinsic factors determine why MSSA may eventually become a MRSA. Furthermore, studies have shown that certain MRSA clones are epidemic while others are only minor clones or sporadic isolates [3]. However, between non-endemic MSSA and epidemic MRSA clones may exist a big genetic distance. Between these two extremes, intrinsic genomic events may occur such as deletions, mutations, recombination and acquirement or loss of additional DNA such as virulence or resistance genes, and possible multiple interactions with coagulase-negative staphylococci. This probably occurs because of exposure to non-favourable environmental stress, most likely the use of antibiotics in and outside the hospital environment. Finally, the genetic constitution of and the interaction with human and animal genetic markers probably also play an important role. Humans and animals may act as a vehicle in transport, transmission and maintenance of the adapted clone.

The endemic MSSA clones may represent some kind of a genetic endpoint, and under the appropriate external stress these clones may become MRSA. However, non-endemic MSSA clones may form a reservoir which after exposure to several external factors, interact with the ubiquitous methicillin-resistant coagulase 
negative staphylococci, and that these clones may sustain an adaptation of its fitness. Therefore non-endemic MSSA clones may need to acquire other eventually non-SCCmec related factors to become genetic stable. It is possible that the actual difference between epidemic HA-MRSA, CA-MRSA and MSSA clones therefore is much less, than one should expect at present and that they are more genetically related but that other markers are to be revealed to proof their relationship. In conclusion, S. aureus continuously evolves and persists in its co-existence with mammals, through genetic adaption, as a response to internal and external threats, which ultimately promotes it survival.

\section{MRSA clones between 2002 and 2006}

The MRSA prevalence in Dutch hospitals is still below $1 \%$, but is rising the last years. This might be due to amongst others patients coming from long-term care facilities, from other countries through cross-border health care and/or through travel. In addition, hospitals near the border with Germany or Belgium often employ HCWs from either side of the border, which are very likely more exposed to potential MRSA carriers. Apart from patients coming from other health care centres, hospitals in general are now faced with CA-MRSA clones and ST398 MRSA associated with livestock farming.

According to the S\&D policy patients belonging to certain MRSA risk groups are always screened on admittance and put in isolation until the results of the MRSA screening are known. Using a stringent isolation and decontamination policy, the chance of transmission and the risk of developing a S. aureus or a MRSA infection will decrease. However, with universal screening more MRSA carriers are detected compared to screening of high risk patients only.

spa typing can be used to show the long-term genetic relationship between MRSA isolates. However, the discriminatory power increases if spa typing in association with another typing method is used. In this study (Chapter 4) the association of spa typing with $\mathrm{SCCmec}$ typing showed the greatest discriminatory power. Furthermore classifying spa types into spa-clonal complexes improves the interpretation of spa typing. MRSA isolates from surveillance cultures from the Euregion showed a great genetic variability of MRSA clones, some of them appeared while others disappeared over time. An explanation might be that some MRSA clones were introduced by health care health exchange cross-border from Belgium and Germany, while others clones were eradicated due to the S\&D policy. Furthermore other clones were previously only seen in Belgium and Germany, like the New York/Japan clone (ST5-MRSA-II), and the South Germany clone (ST228-MRSA-II). After 2005 the UK EMRSA-15 clone (ST22-MRSA-IV) was 
found for the first time in the Netherlands. Different MLST CC5 associated clones were cultured from Belgian health care workers and cystic fibrosis patients, which suggest either import of MRSA or an in vivo transfer of SCCmec to MSSA.

The MSSA isolates in Chapter 3 were from bloodstream infections, while in Chapter 4 the typing of MRSA isolates from surveillance cultures was described. The MSSA isolates may reflect the local genetic pool, while the MRSA may represent import strains. The Paediatric clone (ST5-MRSA-IV) was predominant, however a shift in isolation frequency in this clone was observed from spa type t002 to t447, accompanied with an increased prevalence of resistance to clarithromycin. The difference between the two clones is the deletion of one spa repeat r17 of spa type t002. This may be the consequence of a fitness adaptation of this clone, or to the gradual replacement of the t002 clone by the introduction of a new clone. Other CC5 clones, the UK EMRSA-3 clone, the New York/Japan clone, and the Southern Germany clone were observed, which were not previously observed in the Netherlands. Only $2 \%$ were of the isolates were considered to be CA-MRSA. The strains harbored the PVL gene as well as SCCmec IV and the spa types belonged to ST80 and ST8, two common CA-MRSA lineages found in Europe and the United States respectively. The MRSA population is probably composed of local MRSA clones from hospital and long term facilities, CA-MRSA clones, and MRSA imported possible via health care exchange with Belgium and Germany, or may be through travel.

MRSA and MSSA may adapt to a hostile environment by deletions, genome recombination, mutations or even SCCmec replacements. Comparable to MSSA clones, the importance of non-epidemic minor MRSA clones and sporadic clones need to be further elucidated. Furthermore, in this study, SCCmec of more than $6 \%$ of the MRSA isolates were non-typeable, because unusual genetic combinations were found, which are not yet described. An encounter of MRSA or even MR-CNS strains harboring different SCCmec elements could have led to the formation of a novel SCCmec element, harboring two ccr loci, probably through homologues recombination. It is plausible that many more combinations may exist, either as a permanent or temporary genetic recombination. This may suggest that genetic combinations not only between MRSA clones but also with MSSA and MR-CNS may be even more extensive [1]. Therefore, further research concerning structure of these elements is needed as well as the position of these elements in relation to major and minor clones. One might hypothesise that the local spa types are not only the result of local dissemination but are also reflected in the local antibiotic pressure. Further studies to elucidate this question will be necessary. 


\section{Cross-border dissemination of MRSA}

In the EMR, cross-border health care stimulates the collaboration between the hospitals in the different countries, but is hampered by differences in MRSA prevalence, i.e. in the Netherlands below $1 \%$, in the surrounding countries Belgium and Germany, $23 \%$ and $14 \%$ respectively. With the transfer of patients between health care facilities, there is an increased risk on the introduction of multi-resistant organisms such as MRSA from one health care site to another (Chapter 5). All Dutch isolates were from surveillance cultures, German and Belgian isolates were from clinical specimens of infected patients. SCCmec IV and $\mathrm{SCCmec}$ II predominated in Belgian and German isolates respectively. The fact that MRSA isolates from the Netherlands had a more heterogeneous background, compared to MRSA isolates from Belgium and Germany, suggest indeed that cross-border health care transmission of MRSA has taken place. However clones with SSCmec IV or V and PVL positive, but with a genetic background suggestive for CA-MRSA, as well as the ST398 clone associated with livestock farmers and veterinarians, are also emerging in the Netherlands. The dissemination may be due to the emergence of new local clones or to import through travel abroad, while the dissemination in livestock may be explained by the high antibiotic pressure in livestock farms in all European countries, including the Netherlands. However, so far spreading of CC398 is limited only to persons in close contact with livestock and not to the community [9] Therefore, patients associated with livestock admitted to the hospital are now also screened and pre-emptive isolated. Also in this EMR population a relative high number of clones with nontypeable SCCmec were found, which supports the need for a new SCCmec typing system.

However, concerning health care transmission of MRSA, adjustment of, and applying the same MRSA containment policy in the hospitals of the different countries of the EMR might control spread locally, regionally among patients and HCWs. However in hospitals with a higher MRSA prevalence than in the Netherlands, the logistic capabilities of health care centres does not allow the use of single patient rooms with an ante room under pressure for all MRSA positive patients.

In conclusion, from the results of Chapter 3, 4 and 5 one may conclude that the evolution from non-endemic MSSA clones to highly endemic MRSA clones is a highly dynamic process. Mutual genetic interactions between MSSA, MRSA clones and coagulase negative staphylococci, but also genetic interactions with humans and animals influenced by external factors, such as the use of antibiotics, MSSA and MRSA clones continuously adapt on its fitness. 


\section{Cost of the Search and Destroy policy}

The Netherlands and the Nordic countries have adapted a very successful policy in eradicating and preventing the transmission MRSA in health care centres. However, this puts a considerable financial burden on the hospital finances.

The management of the health care centres need to be convinced, that there is an increased risk in developing an infection when patients are colonised with MRSA, and that MRSA infections are an additional burden to MSSA infections [4]. The potential benefits of a low MRSA prevalence, i.e. the decreased treatment cost of patients and eventually a positive perception towards the public opinion are important issues for health care centres. Initially, the implementation cost of a S\&D policy will be high, in the long run however, the cost associated with the increased morbidity and mortality of patients caused by MRSA will decrease. The amount of cost savings related to implementing a S\&D policy is at present difficult to assess.

In MRSA containment studies, the cost of all disposables, screening cultures, additional HCWs time spent, and decolonisation of the patient are calculated, and this cost is compared with the cost for the treatment of MSSA or MRSA infected patients. However, the studies did not take into account the number of patients who die because of a MRSA infection. It is very difficult to calculate the economical value of a human live. Firstly, patients may die quickly due to a MRSA infection, and therefore, the additional cost is low. As an opposite, the patient might live longer and needs an expensive, closely monitored treatment. Secondly, should one incorporate the value of somebody's economical position in life? What is the value of a farmer, a shop attendant, a judge, a scientist? Thirdly, how is the value of a child, a parent or a grandparent calculated?

Although in studies, screening isolation and decolonisation measures were effective, they often were semi-experimental or non-controlled studies [4, 5]. Nevertheless, the strategy in Dutch and Scandinavian hospitals proved to be very successful. However, further well designed studies are necessary to show the benefit of the S\&D policy, and to demonstrate that it is possible to decrease permanently the MRSA prevalence in hospitals or in at least in certain wards. Studies should preferably show which specific infections control measures have the biggest impact on MRSA containment. On the other hand, studies addressing MRSA containment using less stringent measures are also beneficial such as the use of patient rooms without pressure control. In hospitals with a high MRSA prevalence, it is logistical impossible to isolate these patients in depressurised 
rooms. Eventually, in the future, hospitals may be held responsible for protection of the patients against colonisation by multi-drug organisms.

\section{Rapid detection of MRSA carriers}

Molecular assays may become essential in detecting MRSA in patients admitted to a hospital, because the speed of these assays is much faster than conventional enrichment and culture (Chapter 7). The potential benefits of rapid molecular tests are a rapid detection of MRSA, a decrease in the transmission of MRSA to other patients and HCWs, and a decrease in the number of MRSA infections. However, the total cost of the assays is also much higher than conventional culture, and therefore the use of this test poses a great financial burden on hospitals. This may hamper the introduction of these tests, because a hospital wide introduction may not be cost-effective [10].

Several studies have shown the advantage of the molecular assays in the detection of MRSA in high risk patients. Molecular assays may accurately exclude non-carriers, and may therefore decrease the cost for MRSA containment, because isolation of the patient is discontinued much faster with these assays than with conventional culture $[6,10]$. However, in most hospitals the TAT of these tests is not optimal, because no tests are conducted after office hours and on weekends, thereby reducing the impact of speed. Moreover, the advantage of speed is further reduced when the samples are to be tested in batch.

The sensitivity of molecular assays is between $88 \%$ and $95 \%$. However, in the absence of a valid standard method for comparison interpretation of results is difficult. Molecular assays are only FDA approved for nasal swabs, stored in liquid Stuart's medium, but they appear to be effective with most multiple-site pooled swabs. Furthermore, screening with nasal swabs alone may miss $24 \%$ of MRSA carriers [2, 7]. Samples from non-nasal sites and from multiple-site pooled swabs generally give similar results. However, recently the overall sensitivity of a molecular assay with separately tested multiple site samples was only $84.3 \%$ [6]. Therefore, the application of multiple site pooled samples could increase the sensitivity of the assays. Moreover, sample pooling would also decrease the cost for testing [2]. Alternatively, enrichment before the molecular assays are performed, may further increase the sensitivity, but at the expense of the speed of the assays.

Currently, the PPV of the molecular assays is too low to safely assess the patients as MRSA carriers. When these tests are performed in a hospital population where few MRSA colonisations occur, patients will be unnecessarily exposed to infection 
control measures after a false-positive result, and an increase in the costs for the hospital as a result. Therefore, all positive results in a molecular test should be confirmed by enrichment and culture, while the patient is nursed in isolation.

Molecular assays are much faster in generating a result compared to enrichment and culture. Therefore, these assays may have the potential to decrease the transmission of MRSA among patients and HCWs. To date, some studies have showed a decrease in the transmission, while others failed to show any effect [5]. However, the TAT in these studies was between 20 and 24 hours. Therefore, well designed cross-over studies are needed to show an effect of a rapid result of the molecular essays in the transmission of MRSA. However the compliance of HCWs to infection control measures probably should not be underestimated regarding transmission in comparing detection methods.

The molecular assays are single locus PCR methods which do not detect mecA directly, but amplify the right extremities DNA at the 3' end of the SCCmec at the insertion site adjacent to orfX (MREJ). Furthermore only a limited number of SCCmec types are detected with these assays, and may not detect newly introduced $\mathrm{SCCmec}$. The MREJ region is highly polymorphic, and there are several MREJ variants detected since their first description in 2004 [8]. Hence a regular update of primer and probes to detect all the right extremities of $\mathrm{SCCmec}$ are essential. However, the biggest disadvantage of these assays is the circumstantial detection of the mecA gene. Incomplete SCCmec, deletions in the SCCmec or the mecA region may give false-positive results while the right extremities of SCCmec might still be present. Therefore, the direct amplification of mecA directly in clinical samples would generate less false-positive results. However due to the co-presence of mecA in coagulase negative staphylococci patients materials, this was not possible in clinical samples until recently [11].

In conclusion, molecular assays are faster in generating a result than enrichment and culture, and a negative result may exclude patients as MRSA carriers. However, the PPV in a low prevalence population is unacceptable low, to be used as sole screening test. The main disadvantage of molecular tests is that the molecular assays are more expensive than enrichment and culture, to be used in a hospital wide screening strategy, because these tests may not be cost-effective. Therefore, the use of these assays is likely limited to high risk patients in a high prevalence population. Screening tests are part of MRSA infection control program. Currently, the correct use of infection control measures to contain MRSA is probably more important than the type of screening test used to detect MRSA carriers. 


\section{References}

1. Barbier F, Ruppé $E$, Hernandez D, Lebeaux D, Francois $P$, Felix B, Desprez A, Maiga A, Woerther PL, Gaillard K, Jeanrot C, Wolff M, Schrenzel J, Andremont A, Ruimy R. Methicillin-resistant coagulasenegative staphylococci in the community: high homology of SCCmec Iva between Staphylococcus epidermidis and major clones of methicillin-resistant Staphylococcus aureus. J Infect Dis. 2010;202:270-81.

2. French GL. Methods for screening for methicillin-resistant Staphylococcus aureus carriage. Clin Microbiol Infect. 2009;15(Suppl 7):10-16.

3. Hallin M, Denis O, Deplano A, De Ryck R, Crèvecoeur S, Rottiers S, de Mendonça R, Struelens MJ. Evolutionary relationships between sporadic and epidemic strains of healthcare-associated methicillin-resistant Staphylococcus aureus. Clin Microbiol Infect. 2008;14:659-669.

4. Jarlier V, Trystram D, Brun-Buisson C, Fournier S, Carbonne A, Marty L, Andremont A, Arlet G, Buu-Hoi A, Carlet J, Decré D, Gottot S, Gutmann L, Joly-Guillou MJ, Legrand P, Nicolas-Chanoine $\mathrm{MH}$, Soussy CJ, Wolf M, Lucet JC, Aggoune M, Brücker G, Régnier B; Collégiale de BactériologieVirologie-Hygiène des Hôpitaux Universitaires de l'lle de France. Curbing methicillin-resistant Staphylococcus aureus in 38 French hospitals through a 15-year institutional control program. Arch Intern Med. 2010;170: 552-9.

5. Lucet JC, Regnier B. Screening and decolonisation: does methicillin-susceptible Staphylococcus aureus hold lessons for methicillin-resistant S. aureus? Clin Infect Dis. 2010;51:585-590.

6. Lucke K, Hombach M, Hug M, Pfyffer GE. Rapid detection of methicillin-resistant Staphylococcus aureus (MRSA) in diverse clinical specimens by the BD GeneOhm ${ }^{\mathrm{TM}}$ MRSA assay and comparison with culture. J Clin Microbiol. 2010;48:981-4.

7. Luteijn JM, Hubben GA, Pechlivanoglou P, Bonten MJ, Postma MJ. Diagnostic accuracy of culturebased and PCR-based detection tests for methicillin-resistant Staphylococcus aureus: a metaanalysis. Clin Microbiol Infect. 2010;6.

8. Snyder JW, Munier GK, Heckman SA, Camp P, Overman TL. Failure of the BD GeneOhm ${ }^{\mathrm{TM}}$ StaphSR assay for direct detection of methicillin-resistant and methicillin-susceptible Staphylococcus aureus isolates in positive blood cultures collected in the United States. J Clin Microbiol. 2009;47:3747-3748.

9. van Cleef BA, Verkade.EJM, Wulf MW, Buiting AG, Voss A, Huijsdens XW, van Pelt W, Mulders MN, Kluytmans JA. Prevalence of livestock-associated MRSA in communities with high pigdensities in the Netherlands. PLOS ONE, 2010;5:e9385.

10. Wassenberg MW, Kluytmans JA, Box AT, Bosboom RW, Buiting AG, van Elzakker EP, Melchers WJ, van Rijen MM, Thijsen SF, Troelstra A, Vandenbroucke-Grauls CM, Visser CE, Voss A, Wolffs PF, Wulf MW, van Zwet AA, de Wit GA, Bonten MJ. Rapid screening of methicillin-resistant Staphylococcus aureus using PCR and chromogenic agar: a prospective study to evaluate costs and effects. Clin Microbiol Infect. 2010 (epub ahead of print).

11. Wolk DM, Struelens MJ, Pancholi P, Davis T, Della-Latta P, Fuller D, Picton E, Dickenson R, Denis O, Johnson D, Chapin K. Rapid detection of Staphylococcus aureus and methicillin-resistant S. aureus (MRSA) in wound specimens and blood cultures: multi-center preclinical evaluation of the Cepheid Xpert $^{\text {TM }}$ MRSA/SA skin and soft tissue and blood culture assays. J Clin Microbiol. 2009;47:823-826. 



\section{Chapter 9: Summary}

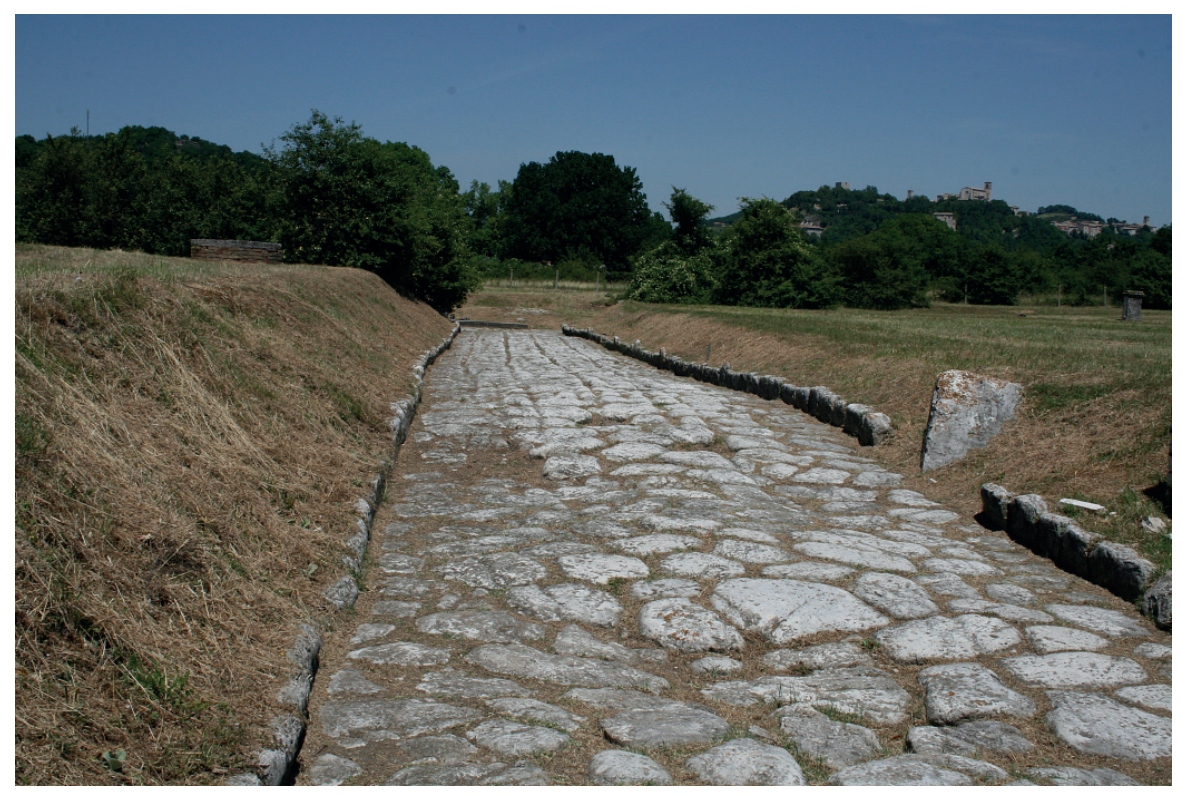

Roman road, Sassoferrato, Italy, 2009 


\section{Summary}

In the Euregion Meuse-Rhine there is an intensive interhospital exchange of patients in and between the different regions in Belgium, Germany and the Netherlands. Therefore the Dutch hospitals are exposed to the import of MRSA colonised patients, because of the higher MRSA prevalence in the surrounding regions. MRSA colonised patients have a higher risk in developing an infection during their hospital stay, and without infection control measures, MRSA may be spread to HCWs and other patients. As a consequence of the worldwide MRSA epidemics in the 80s, the S\&D policy was introduced as a national policy in Dutch hospitals to pro-active screen patients with risk factors for MRSA, to isolate them, and to decontaminate them if necessary.

Chapter 2 gives an overview of current literature of methicillin-susceptible and resistant $S$. aureus in terms of prevalence, risk factors in and outside a hospital, the different typing methods applied for MSSA and MRSA, the MRSA detection methods and finally the cost of MRSA containment.

Molecular typing of 180 MSSA isolates from blood cultures from patients admitted to the University Hospital Maastricht (UHM), between 1999 and 2006, revealed that $50 \%$ have a genetic background common to endemic circulating HA-MRSA types CC5, CC8, CC22, CC30, and CC45, and CA-MRSA types CC1, CC8, CC30, and CC59 (Chapter 3). Since this phenomenon has also been found in other countries, it might imply that these clones can act as recipient for acquiring SCCmec. The other MSSA isolates were not related to MRSA clones, which suggest that MSSA is more genetic heterogeneously than MRSA, and that, certain MSSA clones do not offer the stable genetic environment for inserting SCCmec and thus become MRSA.

Between January 2002 and December 2006, 175 MRSA isolates from surveillance cultures from the Euregion were typed by amplification of the spa gene (Chapter 4). There was a great genetic variability of MRSA clones, some of them appeared while others disappeared. An explanation might be that some MRSA clones were introduced by the health care cross-border exchange from Belgium and Germany, while others clones were eradicated due to the S\&D policy. Until 2005 ST5-MRSAIV (Paediatric clone) with spa types t002 en t447 was predominant in the UHM. Both spa types differed by only deletion of 1 repeat, and it is hypothesised that the Paediatric clone adapted on its fitness, or that a new clone was introduced. Furthermore other clones were previously only seen in Belgium and Germany, like the New York/Japan clone (ST5-MRSA-II), and the South Germany clone (ST228-MRSA-II). After 2005 the UK EMRSA-15 clone (ST22-MRSA-IV) was found 
for the first time. Different MLST CC5 associated clones were cultured from Belgian health care workers and cystic fibrosis patients, which suggest either import of MRSA or an in vivo transfer of SCCmec to MSSA.

The genetic background of 257 MRSA isolates, isolated between July 2005 and April 2006, from infected and colonised patients in the Euregion Meuse-Rhine is described in Chapter 5. Isolates from Dutch hospitals were genetic more divers than those from Belgium and Germany. The Berlin clone (ST45-MRSA-IV) dominated in Belgium, while in Germany the New York/Japan clone (ST5-MRSA-II) is most prevalent. The isolates from Dutch colonised patients however belonged to different clones: the New-York/Japan clone, and the Paediatric clone (ST5MRSA-IV), the EMRSA 2/6 clone (ST8-MRSA-IV) and the Berlin clone were predominant. The increased number of different MRSA clones found, as compared to an earlier study, suggests that exchange and import of MRSA clones has been taken place very likely by cross-border health care.

The cost of the S\&D policy for nursing and treatment of MRSA colonised patients admitted to the University Hospital Maastricht between 2000 and 2004, is presented in Chapter 6, and was compared with the cost of nursing and treatment of MSSA infected patients. Due to an effective S\&D policy the number of MRSA infected patients was below 1 per year, therefore the cost in the MRSA risk group was almost exclusive coming from the MRSA infection control actions and containment. The yearly amount spent on MRSA containment and treatment of $S$. aureus infections was $€ 1,381,200$ and $€ 2,738,128$ respectively. By simulation and variation of the number MRSA colonised patients, we showed that the cost of the S\&D policy did not exceed the cost of treatment of $S$. aureus infections if the number of colonised patients was less than $8 \%$. This means that the S\&D policy is cost-effective, even if the MRSA prevalence is higher compared to the current situation. There have been studies worldwide on the MRSA containment in hospitals or in certain departments. These studies were often different in design, kinds of interventions, and ways to calculate the cost giving different results, showing either absence or presence of any effect. Furthermore only a few publications have appeared on the cost of the S\&D policy in the Netherlands. Therefore well designed studies are necessary to show the effect of the S\&D policy.

The advantage of the recently developed molecular techniques for detecting MRSA in patients, is that the results are available after a few hours, in contrast to the conventional method which includes enrichment and culture and takes at least a few days before getting the results. In Chapter 7, the feasibility of the detection of MRSA with the BD GeneOhm ${ }^{\mathrm{TM}}$ StaphSR and the Cepheid Xpert $^{\mathrm{TM}}$ MRSA assay in a presumptive low MRSA prevalence population is described. Both 
assays have an excellent negative predictive value of $>99 \%$. However the positive predictive value between 31 and $50 \%$ is low, not only due to the low prevalence of MRSA in this study, but also due to false-positive results, which could not be confirmed with culture. A possible explanation is that partial deletions in the mecA or the SCCmec sequences can occur, while the 3' end of SCCmec may be present, and therefore is amplified by the assays. Therefore hospitals with a low prevalence MRSA may be faced with an unnecessary increased cost for isolating patients after a positive result with a molecular assay.

As a consequence of good clinical practice, a positive test result with a molecular assay must always be confirmed by enrichment and culture. To avoid missing true colonised patients, a regular update of primers and probes by the manufacturers of molecular assays is also imperative, in the detection of all types of SCCmec. Hence, rapid molecular assays may have a place in the global containment policy of a hospital, but the assay should be used in conjunction with the infection control policy. Just as well designed studies are inevitable to show any effect on the transmission of MRSA. 


\section{Chapter 10: Samenvatting}

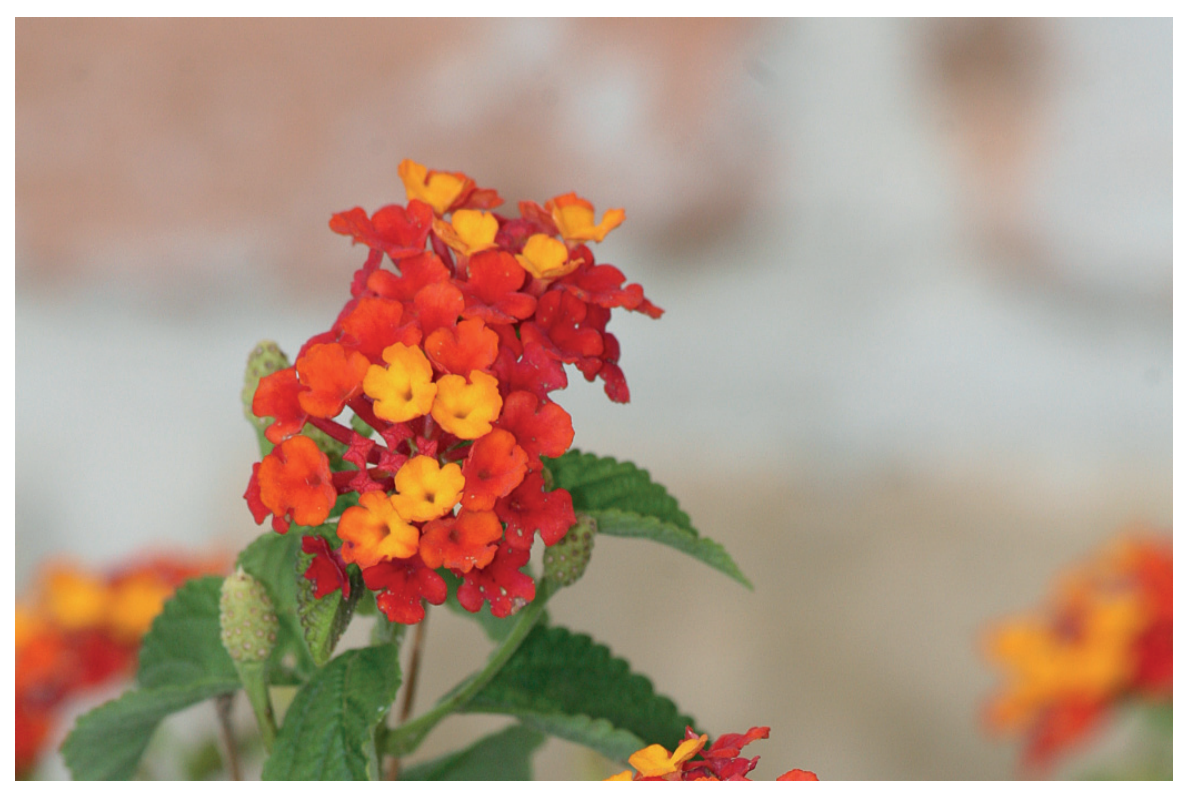

Lantána cámara, Brugge, 2009 


\section{Samenvatting}

In de Euregio Maas-Rijn is er een intensieve uitwisseling van patiënten tussen de verschillende ziekenhuizen in België, Duitsland en Nederland. Dit betekent dat Nederlands-Limburgse ziekenhuizen geconfronteerd kunnen worden aan met MRSA gekoloniseerde patiënten die afkomstig zijn uit ziekenhuizen van de omringende Euregio's waar de MRSA kolonisatiegraad van patiënten hoger is dan in Nederland. MRSA gekoloniseerde patiënten hebben een verhoogd risico om tijdens hun verblijf in een ziekenhuis een infectie met een (MR)SA te ontwikkelen en om, indien geen preventieve maatregelen worden genomen, MRSA te verspreiden naar andere patiënten en gezondheidsmedewerkers. Ten gevolge van wereldwijde MRSA epidemieën in de jaren ' 80 werd de 'Search en Destroy policy' (S\&D beleid) in Nederlandse ziekenhuizen ingevoerd om de verspreiding van MRSA tegen te gaan en alle potentieel gekoloniseerde patiënten proactief op MRSA te screenen en dragers te isoleren en indien nodig te dekoloniseren.

Hoofdstuk 2 geeft een overzicht van de huidige literatuur over methicillinegevoelige en -resistente Staphylococcus aureus voor wat betreft prevalentie, risico factoren binnen en buiten een ziekenhuis, de verschillende typeringsmethoden van (MR)SA, de detectiemethoden van MRSA en de kosten die nodig zijn om de verspreiding van MRSA in ziekenhuizen te beheersen.

Moleculaire typering van 180 MSSA isolaten uit bloedkweken afkomstig van patiënten opgenomen in het Universitair Ziekenhuis Maastricht (UZM) tussen 1999 en 2006 (hoofdstuk 3), toonde aan dat 50\% van de isolaten een genetische achtergrond had die vergelijkbaar was met de endemisch circulerende HA-MRSA types, CC5, CC8, CC22, CC30 en CC45 en de CA-MRSA types, CC1, CC8, CC30 en CC59. Dit werd ook in andere landen waargenomen, zodat aangenomen kon worden dat deze klonen relatief gemakkelijk SCCmec konden verwerven. De overige MSSA isolaten waren genetisch niet geassocieerd met MRSA klonen, wat suggereerde dat MSSA genetisch meer heterogeen is dan MRSA, en dat sommige MSSA klonen bijgevolg geen stabiele genetische omgeving bieden om SCCmec te verwerven en dus om zelf MRSA te worden.

Tussen januari 2002 en december 2006 werden 175 MRSA isolaten afkomstig van surveillance kweken uit de Euregio, door amplificatie van het spa gen, getypeerd (Hoofdstuk 4). Er was een grote genetische verscheidenheid van MRSA klonen, sommigen verschenen, terwijl andere klonen in deze periode verdwenen. Een mogelijk verklaring kan zijn dat sommige MRSA klonen door de grensoverschrijdende gezondheidszorg vanuit België en Duitsland werden geïntroduceerd, terwijl andere klonen door het S\&D beleid werden geëlimineerd. 
Tot 2005 was ST5-MRSA-IV ('Paediatric clone'), met spa types t002 en t447, predominant in het UZM. Beide spa types verschilden slechts door 1 'repeat'. De hypothese was dat er ofwel een aanpassing van de bacteriële "fitheid" had plaatsgevonden ofwel dat er een nieuwe kloon in de populatie geïntroduceerd was. Verder werden sommige klonen enkel in België en Duitsland waargenomen, zoals de New York/Japan kloon (ST5-MRSA-II) en de Zuid-Duitse kloon (ST228MRSA-II). Na 2005 werd de UK EMRSA-15 kloon (ST22-MRSA-IV) voor het eerst in Nederland gevonden. Verschillende MLST CC5 geassocieerde klonen werden geïsoleerd bij Belgische gezondheidswerkers en mucoviscidose patiënten, wat ofwel import van MRSA ofwel een in vivo transfer van SCCmec naar een MSSA veronderstelde.

Van 257 MRSA isolaten, afkomstig van patiënten uit de Euregio Maas-Rijn die geïnfecteerd of gekoloniseerd waren in de periode tussen juli 2005 en april 2006, werd hun genetische achtergrond bepaald (Hoofdstuk 5). De MRSA isolaten uit Nederland waren genetisch meer verschillend dan isolaten uit België en Duitsland. In België werd vooral de Berlijn kloon (ST45-MRSA-IV) gevonden, terwijl in Duitsland de New York/Japan kloon (ST5-MRSA-II) domineerde. De isolaten van gekoloniseerde Nederlandse patiënten behoorden tot verschillende klonen: hoofdzakelijk werden de New-York/Japan kloon, de 'Paediatric clone' (ST5-MRSA-IV), de UK EMRSA 2/6 kloon (ST8-MRSA-IV) en de Berlijn kloon gevonden. De stijging van het aantal verschillende klonen die gevonden werden in vergelijking met een eerdere studie, doet vermoeden dat uitwisseling en import van MRSA klonen door grensoverschrijdende gezondheidszorg heeft plaats gevonden.

De kosten van het S\&D beleid, de verpleging en de behandeling van MRSA gekoloniseerde patiënten die opgenomen waren in het UZM tussen 2000 en 2004 werden vergeleken met de kosten van de verpleging en behandeling van infecties bij patiënten door MSSA, zijn beschreven in hoofdstuk 6. Door het effectieve S\&D beleid waren er bijna geen MRSA geïnfecteerde patiënten (0,4/jaar), en zijn de kosten in de MRSA groep vooral afkomstig van preventieve maatregelen. Deze zijn met name gericht op het tegen gaan van de verspreiding van MRSA naar andere patiënten en gezondheidsmedewerkers. Het totale bedrag dat jaarlijks besteed werd aan MRSA preventie en aan de preventie en behandeling van $S$. aureus infecties, bedroeg $€ 1.381 .200$ respectievelijk $€ 2.738 .128$. Door een simulatie van het aantal opgenomen en gekoloniseerde patiënten kon worden aangetoond dat de S\&D kosten lager waren dan de behandelingskosten wanneer het aantal MRSA gekoloniseerde patiënten onder de 8\% bleef. Er zijn wereldwijd verschillende publicaties geweest over infectiepreventieve interventies in ziekenhuizen of op bepaalde afdelingen van ziekenhuizen. De studies zijn vaak 
verschillend van opzet, met verschillende soorten interventies, andere berekeningswijze van de kosten en verschillende wijze van presentatie van de effecten. Er zijn ook maar enkele publicaties verschenen over de kosten van het S\&D beleid in Nederland. Daarom is een goed opgezette studie noodzakelijk om het effect van het S\&D beleid aan te tonen.

De recent ontwikkelde moleculaire technieken hebben het voordeel dat het resultaat van de 'screening' van potentiële MRSA dragers binnen enkele uren bekend wordt, dit in tegenstelling met de conventionele methode met behulp van aanrijking en kweek, waar pas na 3 tot 5 dagen het resultaat verkregen wordt.

De bruikbaarheid met betrekking tot de opsporing van MRSA met de BD GeneOhm ${ }^{\mathrm{TM}}$ StaphSR en de Cepheid ${ }^{\mathrm{TM}}$ Xpert MRSA assay, in een (vermoedelijk) lage prevalentie MRSA populatie werd beschreven in hoofdstuk 7. Beide moleculaire testen hebben een uitstekende negatieve voorspellende waarde van $>99 \%$. De positieve voorspellende waarde van deze testen, tussen $31 \%$ en $50 \%$ is echter laag doordat de positieve resultaten met de moleculaire technieken niet met kweek bevestigd konden worden. Een mogelijke verklaring hiervoor is dat gedeeltelijke deleties in de mecA regio en SCCmec sequenties kunnen optreden, maar waarbij het 3' uiteinde van SCCmec nog aanwezig is en geamplifieerd wordt. Door de relatief lage positief voorspellende waarde van de moleculaire testen worden ziekenhuizen met een lage prevalentie van MRSA, geconfronteerd met onnodige kosten vanwege de isolatie van patiënten, die positief zijn met de moleculaire methode. Een positief resultaat met een moleculaire test dient derhalve altijd met aanrijking en kweek bevestigd te worden. Om te vermijden dat werkelijk gekoloniseerde patiënten gemist worden, is een regelmatige update van primers en probes door de fabrikanten van de moleculaire testen noodzakelijk. Moleculaire testen kunnen een rol spelen in het infectie preventie beleid met betrekking tot MRSA van een ziekenhuis, echter alleen als onderdeel van het algemene infectie preventie beleid. Het effect van deze testen op de transmissie en verspreiding van MRSA zal eveneens verder onderzocht moeten worden. 


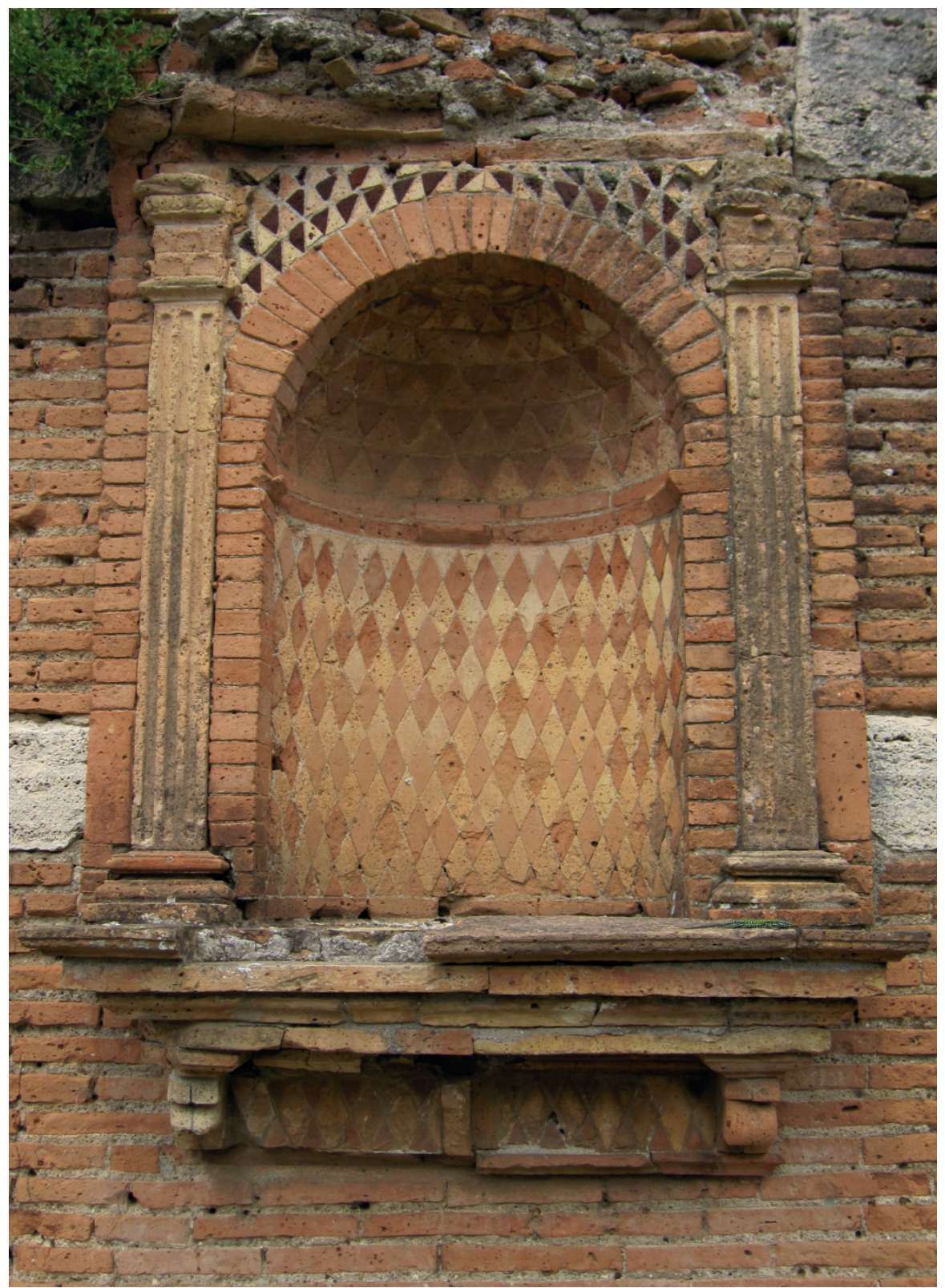

Ostia Antica, 2010 



\section{Acknowledgements (dankwoord)}

Toen ik in 2005 uit het Universitair Ziekenhuis Maastricht vertrok en aan een nieuwe baan in het Algemeen Ziekenhuis Sint-Jan in Brugge begon, leek een combinatie van wetenschappelijk onderzoek naast mijn werk als artsmicrobioloog een leuke afwisseling. Zoals verwacht nam de leuke afwisseling al snel veel vrije tijd in beslag, maar ik ben tevreden met het resultaat, het is de moeite waard geweest. Ik was daarom ook vereerd dat Cathrien Bruggeman mijn promotor wilde zijn en ik wil haar bedanken voor het vertrouwen en de steun.

Ik ben altijd bedachtzaam geweest met alles wat ik deed. Ik behaalde relatief laat mijn rijbewijs, ik kocht pas op latere leeftijd mijn eerste auto, ik ontmoette mijn vrouw, Josine, toen de meeste mensen van mijn leeftijd al mama of papa waren. Dus begon ik ook op latere leeftijd aan de mijn studies geneeskunde. Mijn goede vriend Rick toonde mij dat je met geestdrift, motivatie en natuurlijk goede muziek, veel kan bereiken. De combinatie van studeren en werken als analist waren niet gemakkelijk en ik ben daarom veel dank verschuldigd aan mijn toenmalige baas Raymond Vanhoof van het Pasteur Instituut. Dankzij de steun van Professor dr. Sabine Lauwers van de Vrije Universiteit Brussel kon ik specialiseren in de Klinische Biologie en begon ik dus aan een nieuwe periode in mijn leven. In wilde me verder in microbiologie verdiepen, maar om uiteindelijk als arts-microbioloog te werken, waren er meer mogelijkheden in Nederland...and out the door I went.

Dit proefschrift schrijven was dus een logisch gevolg van mijn manier van leven. Het vooruitzicht dat ik aan het einde van dit proefschrift 51 jaar oud zou zijn heeft geen invloed op mij gehad. Leeftijd heeft voor mij nooit meegespeeld in mijn beslissingen, ik moet nu alleen langer recupereren na sporten en ik moet alles minstens 2 maal lezen.

Maar dit project is ook gekomen dank zij de inzet van verschillende mensen. Op de eerste plaats wil ik Ellen Stobberingh bedanken, we kunnen heel goed met elkaar opschieten. Ze was steeds zeer geduldig, wist ze mij in de juiste richting te sturen, moedigde me aan, beschikt over een ongelooflijke kennis, en maakte ze mij regelmatig duidelijk wat belangrijk was en wat niet, of om het in de woorden van Josine te zeggen: "Ellen zit weer achter je veren aan".

Ik had het geluk dat Bart Gordts mijn collega werd in Brugge, hij was onmiddellijk gewonnen om mijn onderzoek verder uit te werken. Zijn steun en zijn kennis hebben mij enorm geholpen. Ik zal hem in ieder geval missen nu hij naar 't Stad vertrokken is. Veel succes Bart, in Antwerpen.

Mijn speciale dank gaat uit naar Ruud Deurenberg, die uitstekend werk leverde voor, tijdens en na de typeringen van de isolaten. Ik heb kunnen profiteren van 
zijn enorme kennis over MRSA, want het was voor mij toch even doorbijten om al de typeringstechnieken te kunnen begrijpen en interpreteren. Daarom gaat mijn dank gaat eveneens uit naar Patrick Beisser die resultaten van typeringen op een eenvoudige manier kon verklaren. Ook mag ik Ellen Broex niet vergeten die een hele kluif had aan het verzamelen en het verwerken van de kosten met betrekking tot MRSA preventie in het Universitair Ziekenhuis Maastricht.

Mijn dank gaat uit naar mijn collega's in Brugge: Marijke Reynders en Dirk Bernard voor hun steun en ideeën. Ook de analisten van het labo Microbiologie van het AZ Sint-Jan, en in het bijzonder Patrick Descheemaeker, wil ik danken en hoop nog vele plezante jaren met hun te mogen samenwerken.

Liefste Josine, toen je zei dat je aan de lay-out wilde werken, was ik heel tevreden, want ik wist dat het proefschrift perfect zou verbeterd en afgewerkt worden. Soms tot mijn wanhoop, hield je alles strikt onder controle (de border collie in jou: "Eric, waarom staat er mecA en niet mecA?"), maar door je geduld, je enthousiasme en je positieve ingesteldheid, werd het heel mooi geheel.

Ik wil verder alle mensen bedanken die rechtstreeks of onrechtstreeks hebben meegewerkt aan het tot stand brengen van deze thesis.

En last but not least, wil ik m'n ouders bedanken, die het inzicht hadden en me de mogelijkheden gaven om verder te studeren.

Eric

4 november 2010 


\section{Curriculum Vitae}

Eric Nulens ( ${ }^{\circ}$ Hasselt, 24/7/1959), koos al tijdens zijn middelbare schoolopleiding voor het laboratoriumwerk en studeerde in 1978 af als labtechnicus. Tijdens zijn hogere studies behaalde hij een A1-diploma in farmaceutische en biologische

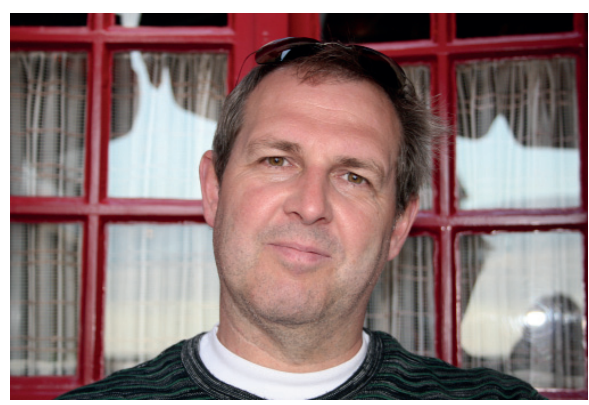
technieken. Waarna er in 1982 een specialisatiejaar in milieusanering volgde.

Hij werkte jaren als gediplomeerd analist in microbiologische laboratoria van verschillende Brusselse ziekenhuizen en aan het Instituut Pasteur van Brussel.

Op zijn $27^{\text {ste }}$ ving hij zijn artsenopleiding aan aan de Vrije Universiteit Brussel en dit voor onder andere de middenjury in combinatie met zijn werk als analist. Zijn inzet werd beloond en hij studeerde af - met onderscheiding - in 1996, en is hij zo een van de weinige Belgen die arts werd via deze middenjury.

In 1996 startte hij zijn opleiding tot klinisch bioloog in het Algemeen Ziekenhuis Sint-Jan, Brugge, het Academisch Ziekenhuis van zijn alma mater en aan de Cliniques Universitares, Université Catolique de Louvain, Saint-Luc, Brussel. Natuurlijk hield het studeren hem in de greep en zo behaalde hij in 2000 ook nog een diploma als ziekenhuishygiënist en in 2001 het diploma voor medische en veterinaire mycologie aan het Tropische Instituut van Antwerpen.

$\mathrm{Na}$ het einde van zijn specialisatie en erkenning in 2001 als klinisch bioloog, vertrok hij voor zijn eerste stappen in zijn nieuwe vakgebied naar het Nijmeegs Universitair Centrum voor Infectieziekten, Sint-Radboud. Zijn erkenning van artsmicrobioloog bij het BIG-register verkreeg hij in 2002.

In 2004 had hij de kans om aan het Academisch Ziekenhuis Maastricht als medisch microbioloog te werken, dus 'net over de grens' ('across-border'). In deze hoedanigheid had hij een speciale interesse in ziekenhuishygiëne en infectiepreventie. Hij maakte kennis met de Nederlandse aanpak van het MRSAprobleem (MRSA: methicillin-resistant Staphylococcus aureus) die erg verschillend is van het Belgische beleid.

Sinds 2005 combineert hij in de Medische Microbiologie van het Algemeen Ziekenhuis Sint-Jan Brugge zijn taak als klinisch bioloog met het werken aan zijn 
proefschrift. In het AZ St.-Jan wil hij de positieve elementen van het Nederlandse laboratoriumbeleid koppelen met het Belgische pragmatisme en natuurlijk de MRSA-infectie druk zo laag mogelijk te houden.

Eric Nulens is auteur en medeauteur van meerdere wetenschappelijke publicaties in internationale tijdschriften en boeken. Hij houdt zich op de hoogte van de laatste ontwikkelingen via zelfstudie en deelname aan congressen. Via posters communiceert hij op regelmatige basis zijn bevindingen aan zijn collega's.

In 2001 trouwde hij met Josine Bielen. Het vaderschap ging aan hem voorbij, maar hij is baas van 2 vrolijke Engelse Cocker Spaniëls: Edvard en Trixi. 


\section{Publications}

1. Nulens E, Descheemaeker P, Deurenberg RH, Stobberingh EE, Gordts B. Contribution of two molecular assays as compared to selective culture for MRSA screening in a low MRSA prevalence population. Infection. 2010;38:98-101. Erratum in: Infection. 2010;38:345.

2. Deurenberg RH, Nulens E, Valvatne H, Sebastian S, Driessen C, Craeghs J, De Brauwer E, Heising B, Kraat YJ, Riebe J, Stals FS, Trienekens TA, Scheres J, Friedrich AW, van Tiel FH, Beisser PS, Stobberingh EE. Cross-border dissemination of methicillin-resistant Staphylococcus aureus, Euregio MeuseRhin region. Emerg Infect Dis. 2009;15:727-34.

3. Nulens E, Stobberingh EE, Smeets E, van Dessel H, Welling MA, Sebastian S, van Tiel FH, Beisser PS, Deurenberg RH. Genetic diversity of methicillinresistant Staphylococcus aureus in a tertiary hospital in The Netherlands between 2002 and 2006. Eur J Clin Microbiol Infect Dis. 2009;28:631-9.

4. Nulens E, Broex E, Smeets E, Deurenberg R, Stobberingh E. MRSA management: a cost efficiency problem in crossborder hospitals? ICU management. 2008;8:18-19.

5. Stobberingh E, Nulens E. MRSA management. Hospital. 2008;10:33-34.

6. Nulens E, Stobberingh EE, van Dessel H, Sebastian S, van Tiel FH, Beisser PS, Deurenberg RH. Molecular characterisation of Staphylococcus aureus bloodstream isolates collected in a Dutch University Hospital between 1999 and 2006. J Clin Microbiol. 2008;46:2438-41.

7. Nulens E, Broex E, Ament A, Deurenberg RH, Smeets E, Scheres J, van Tiel FH, Gordts B, Stobberingh EE. Cost of the meticillin-resistant Staphylococcus aureus search and destroy policy in a Dutch university hospital. $J$ Hosp Infect. 2008;68:301-7.

8. Smismans A, Goossens VJ, Nulens E, Bruggeman CA. Comparison of five different immunoassays for the detection of Borrelia burgdorferi IgM and IgG antibodies. Clin Microbiol Infect. 2006;12:648-55.

9. Nulens E, De Laere E, Vandevelde H, Hilbrands LB, Rijs AJ, Melchers WJ, Verweij PE. Alternaria infectoria phaeohyphomycosis in a renal transplant patient. Med Mycol. 2006;44:379-82. 
10. Voss A, Nulens E. Laboratory Areas. In: A Guide to Infection Control in the Hospital. Third edition. Wenzel . P., Brewer T. F., Butzler J.-P. eds. ISID, Boston, 2005.

11. Nulens E, Voss A, Gould I, Mackenzie F, Deplano A, Cookson B, Alp E, Bouza E. Staphylococcus aureus carriage among participants at the $13^{\text {th }}$ European Congress of Clinical Microbiology and Infectious Diseases. Eur J Clin Microbiol Infec Dis. 2005;24:145-148.

12. Voss A, Nulens E. Prevention and control of laboratory-acquired infections. In: Manual of Clinical Microbiology, Eight edition. Murray P. R., Baron E. J., Jorgensen J. H. Pfaller M. A., Yolken R. H. eds. American Society for Microbiology, Washington DC, 2003, 109-120.

13. Nulens E, Eggink C, Rijs TMMA, Wesseling P, Verweij PE. Keratitis by Scedosporium apiospermum succesfully treated with voriconazole and penetrating keratoplasty. J Clin Microbiol. 2003;41:2261-2264.

14. Nulens E, Eggink C, Verweij P. Combination therapy for keratitis by the fungus Scedosporium. Cornea. 2003;22:92.

15. Nulens E, Voss A. Laboratory diagnosis and biosafety issues of biological warfare agents. Clin Microb Infect. 2002;8:455-466.

16. Nulens E, Bussels B, Bols A, Gordts B, Van Landuyt HW. Recurrent bacteremia by Chryseobacterium indologenes in an oncology patient with a totally implanted intravascular device. Clin Microb Infect. 2001;7:391-393.

17. Nulens $E$, Bodéus $M$, Bonelli $F$, Soleti $A$, Goubau P. Reactivity to $p 52$ and CM2 recombinant proteins in primary human cytomegalovirus infection with a microparticle agglutination assay. Clin Diagn Lab Immunol. 2000;7:536-539.

18. Nulens E, Bernard D, Blaton V. Homogene (indirecte) HDL-cholesterol en LDLcholesterol bepalingen. Tijdschrift van de Belgische Vereniging van Laboratorium Technologen. 1998;25:403-413.

19. Vanhoof R, Nulens E, Nyssen HJ, Hannecart-Pokorni E. The utility of the PCR method to study aacA gene heterogeneity in Serratia marcescens. J Antimicrob Chemother. 1994;34:595-596. 
20. Vanhoof R, Content J, Van Bossuyt E, Nulens E, Sonck P, Depuydt F, Hubrechts JM, Maes $P$, Hannecart-Pokorni E. Use of the polymerase chain reaction (PCR) for the detection of aacA genes encoding aminoglycoside- 6 ' $\mathrm{N}$-acetyltransferases in reference strains and Gram-negative clinical isolates from two Belgian Hospitals. J Antimicrob Chemother. 1993;32:23-35.

21. Vanhoof R, Godard C, Nulens E, Nyssen HJ, Wildemauwe C, Hubrechts JM, Maes $P$, Hannecart-Pokorni E. Serotypes and extended spectrum beta-lactam resistance in aminoglycoside resistant Pseudomonas aeruginosa isolates from two Belgian general hospitals: a seven year study. Hosp Infect. 1993;24:129-138.

22. Vanhoof R, Nulens E, Nyssen HJ, Hannecart-Pokorni E. Comparative in vitro activity of cefepime and four extended-spectrum beta-lactams on 1251 aminoglycoside-resistant Gram-negative hospital strains. Chemother. 1992;38:225-231.

23. Vanhoof R, Nyssen HJ, Nulens E, Roebben E, Hubrechts JM. Activité antibactérienne du carumonam et du cefpirome sur des souches hospitalières résistantes à la gentamicine et la céfalotine: comparaison avec d'autres bêta-lactamines, de nouvelles fluoroquinolones, aminoglycosides et d'autre d'antibiotiques divers. Path Biol. 1990;38:634-637.

24. Vanhoof R, Hubrechts JM, Nyssen HJ, Nulens E, Leger J, De Schepper N, Kupperberg E, Couvreur ML. Activity of cefotiam in combination with betalactam antibiotics on enterobacterial hospital strains. Pharm Weekbl. (Sci). 1990;12:104-106.

25. Vanhoof R, Hubrechts JM, Roebben E, Nyssen HJ, Nulens E, Leger J, De Schepper N. The comparative activity of pefloxacin, enoxacin, ciprofloxacin and 13 other antimicrobial agents against enteropathogenic microorganisms. Infection. 1986;14:294-298.

26. Vanhoof R, Hubrechts JM, Roebben E, Claeys M, Nulens E, Nyssen HJ, Vanhaeverbeek M, Van Gysel JP. A one year survey on the epidemiology of aminoglycoside resistance in a general hospital: influence of amikacin. Acta Clin Belg. 1986;41:248-254. 